\title{
HAT-P-65b AND HAT-P-66b: TWO TRANSITING INFLATED HOT JUPITERS AND OBSERVATIONAL EVIDENCE FOR THE REINFLATION OF CLOSE-IN GIANT PLANETS*
}

\author{
J. D. Hartman ${ }^{1}$, G. Á. Bakos ${ }^{1,14,15}$, W. Bhatti ${ }^{1}$, K. Penev $^{1}$, A. Bieryla ${ }^{2}$, D. W. Latham ${ }^{2}$, G. Kovács ${ }^{3}$, \\ G. Torres ${ }^{2}$, Z. Csubry ${ }^{1}$, M. de Val-Borro ${ }^{1}$, L. Buchhave ${ }^{4}$, T. Kovács ${ }^{3}$, S. Quinn ${ }^{5}$, A. W. Howard ${ }^{6}$, \\ H. IsaACSON ${ }^{7}$, B. J. Fulton ${ }^{6}$, M. E. EveretT ${ }^{8}$, G. Esquerdo ${ }^{2}$, B. BéKY ${ }^{13}$, T. SzKLEnar ${ }^{9}$, E. FalCo $^{2}$, \\ A. Santerne ${ }^{11}$, I. Boisse ${ }^{10}$, G. Hébrard ${ }^{12}$, A. Burrows ${ }^{1}$, J. LÁZár ${ }^{9}$, I. PaPP ${ }^{9}$, ANd P. SÁri ${ }^{9}$ \\ ${ }^{1}$ Department of Astrophysical Sciences, Princeton University, Princeton, NJ 08544, USA; jhartman@ astro.princeton.edu \\ ${ }^{2}$ Harvard-Smithsonian Center for Astrophysics, Cambridge, MA 02138, USA \\ ${ }^{3}$ Konkoly Observatory of the Hungarian Academy of Sciences, Budapest, Hungary \\ ${ }^{4}$ Centre for Star and Planet Formation, Natural History Museum of Denmark, University of Copenhagen, DK-1350 Copenhagen, Denmark \\ ${ }_{5}$ Department of Physics and Astronomy, Georgia State University, Atlanta, GA 30303, USA \\ ${ }^{6}$ Institute for Astronomy, University of Hawaii, Honolulu, HI 96822, USA \\ ${ }^{7}$ Department of Astronomy, University of California, Berkeley, CA, USA \\ ${ }^{8}$ National Optical Astronomy Observatory, Tucson, AZ, USA \\ ${ }^{9}$ Hungarian Astronomical Association, Budapest, Hungary \\ Hungarian Astronomical Association, Budapest, Hungary
${ }^{10}$ Aix Marseille Université, CNRS, LAM (Laboratoire d'Astrophysique de Marseille) UMR 7326, F-13388, Marseille, France \\ ${ }^{11}$ Instituto de Astrofisica e Ciências do Espaço, Universidade do Porto, CAUP, Rua das Estrelas, PT4150-762 Porto, Portugal \\ ${ }^{12}$ Institut d'Astrophysique de Paris, UMR7095 CNRS, Université Pierre \& Marie Curie, 98bis boulevard Arago, F-75014 Paris, France \\ ${ }^{3}$ Google, Googleplex, 1600 Amphitheatre Parkway, Mountain View, CA 94043, USA \\ Received 2016 July 15; revised 2016 September 7; accepted 2016 September 9; published 2016 November 23
}

\begin{abstract}
We present the discovery of the transiting exoplanets HAT-P-65b and HAT-P-66b, with orbital periods of 2.6055 and 2.9721 days, masses of $0.527 \pm 0.083 M_{\mathrm{J}}$ and $0.783 \pm 0.057 M_{\mathrm{J}}$, and inflated radii of $1.89 \pm 0.13 R_{\mathrm{J}}$ and $1.59_{-0.10}^{+0.16} R_{\mathrm{J}}$, respectively. They orbit moderately bright $(V=13.145 \pm 0.029$ and $V=12.993 \pm 0.052)$ stars of mass $1.212 \pm 0.050 M_{\odot}$ and $1.255_{-0.054}^{+0.107} M_{\odot}$. The stars are at the main-sequence turnoff. While it is well known that the radii of close-in giant planets are correlated with their equilibrium temperatures, whether or not the radii of planets increase in time as their hosts evolve and become more luminous is an open question. Looking at the broader sample of well-characterized close-in transiting giant planets, we find that there is a statistically significant correlation between planetary radii and the fractional ages of their host stars, with a false-alarm probability of only $0.0041 \%$. We find that the correlation between the radii of planets and the fractional ages of their hosts is fully explained by the known correlation between planetary radii and their present-day equilibrium temperatures; however, if the zero-age main-sequence equilibrium temperature is used in place of the present-day equilibrium temperature, then a correlation with age must also be included to explain the planetary radii. This suggests that, after contracting during the pre-main-sequence, close-in giant planets are reinflated over time due to the increasing level of irradiation received from their host stars. Prior theoretical work indicates that such a dynamic response to irradiation requires a significant fraction of the incident energy to be deposited deep within the planetary interiors.
\end{abstract}

Key words: stars: individual (HAT-P-65, GSC 1111-00383, HAT-P-66, GSC 3814-00307) techniques: photometric - techniques: spectroscopic

Supporting material: machine-readable table

\footnotetext{
* Based on observations obtained with the Hungarian-made Automated Telescope Network. Based on observations obtained at the W. M. Keck Observatory, which is operated by the University of California and the California Institute of Technology. Keck time has been granted by NOAO (A289Hr, A245Hr) and NASA (N029Hr, N154Hr, N130Hr, N133Hr, N169Hr, $\mathrm{N} 186 \mathrm{Hr}$ ). Based on observations obtained with the Tillinghast Reflector $1.5 \mathrm{~m}$ telescope and the $1.2 \mathrm{~m}$ telescope, both operated by the Smithsonian Astrophysical Observatory at the Fred Lawrence Whipple Observatory in Arizona. Based on observations made with the Nordic Optical Telescope, operated by the Nordic Optical Telescope Scientific Association at the Observatorio del Roque de los Muchachos, La Palma, Spain, of the Intituto de Astrofísica de Canarias. Based on observations made with the SOPHIE spectrograph on the $1.93 \mathrm{~m}$ telescope at Observatoire de Haute-Provence (OHP, CNRS/AMU), France (programs 15A.PNP.HEBR and 15B.PNP. HEBR). Data presented herein were obtained at the WIYN Observatory from telescope time allocated to NN-EXPLORE through the scientific partnership of the National Aeronautics and Space Administration, the National Science Foundation, and the National Optical Astronomy Observatory. This work was supported by a NASA WIYN PI Data Award, administered by the NASA Exoplanet Science Institute.

14 Alfred P. Sloan Research Fellow.

15 Packard Fellow.
}

\section{INTRODUCTION}

The first transiting exoplanet (TEP) discovered, HD 209458b (Charbonneau et al. 2000; Henry et al. 2000), surprised the community in having a radius much larger than expected based on theoretical planetary structure models (e.g., Burrows et al. 2000; Bodenheimer et al. 2001). Since then, many more inflated transiting planets have been discovered, the largest being WASP-79b with $R_{P}=2.09 \pm 0.14 R_{\mathrm{J}}$ (Smalley et al. 2012). It has also become apparent that the degree of planet inflation is closely tied to a planet's proximity to its host star (e.g., Fortney et al. 2007; Kovács et al. 2010; Béky et al. 2011; Enoch et al. 2011a, 2012). This is expected on theoretical grounds, as some additional energy, beyond the initial heat from formation, must be responsible for making the planet so large, and in principle there is more than enough energy available from stellar irradiation or tidal forces to inflate close-in planets at $a<0.1$ au (Bodenheimer et al. 2001). Whether and how the energy is transfered into planetary 
interiors remains a mystery, however, despite a large amount of theoretical work devoted to the subject (see, e.g., Spiegel \& Burrows 2013, for a review). The problem is intrinsically challenging, requiring the simultaneous treatment of molecular chemistry, radiative transport, and turbulent (magneto)hydrodynamics, carried out over pressures, densities, temperatures, and length scales that span many orders of magnitude. Theoretical models of planet inflation have thus, by necessity, made numerous simplifying assumptions, often introducing free parameters whose values are unknown, or poorly known. One way to make further progress on this problem is to build up a larger sample of inflated planets to identify patterns in their properties that may be used to discriminate between different theories.

Recently Lopez \& Fortney (2016) proposed an observational test to distinguish between two broad classes of models. Noting that once a star leaves the main sequence the irradiation of its planets with periods of tens of days becomes comparable to the irradiation of very short period planets around main-sequence stars, they suggested searching for inflated planets with periods of tens of days around giant stars. Planets at these orbital periods are not inflated when found around main-sequence stars (Demory \& Seager 2011), so finding them to be inflated around giants would indicate that the enhanced irradiation is able to directly inflate the planets. As shown, for example, by Liu et al. (2008) and also by Spiegel \& Burrows (2013), this in turn would imply that energy must be transferred deep into the planetary interior, and would rule out models where the energy is deposited only in the outer layers of the planet and serves simply to slow the planet's contraction from its initial highly inflated state. The recently discovered planet EPIC 211351816.01 (Grunblatt et al. 2016, found using K2) is a possible example of a reinflated planet around a giant star, with the planet having a larger-than-usual radius of $1.27 \pm 0.09 R_{\mathrm{J}}$ given its orbital period of 8.4 days. The planet K2-39b (Van Eylen et al. 2016), on the other hand, does not appear to be exceptionally inflated $\left(R_{p}=0.732 \pm 0.098 R_{\mathrm{J}}\right)$ despite being found on a very short period orbit around a subgiant star. This planet, however, is in the super-Neptune mass range $\left(M_{p}=0.158 \pm 0.031 M_{\mathrm{J}}\right)$ and may not have a gas-dominated composition.

Here we present the discovery of two transiting inflated planets by the Hungarian-made Automated Telescope Network (HATNet; Bakos et al. 2004). As we will show, the planets have radii of $1.89 \pm 0.13 R_{\mathrm{J}}$ and $1.59_{-0.10}^{+0.16} R_{\mathrm{J}}$ and are around a pair of stars that are leaving the main sequence. HATNet, together with its southern counterpart HATSouth (Bakos et al. 2013), has now discovered 17 highly inflated planets with $R \geqslant 1.5 R_{\mathrm{J}}{ }^{16}$ Adding those found by WASP (Pollacco et al. 2006), Kepler (Borucki et al. 2010), Tillinghast Reflector Echelle Spectrograph (TrES; e.g., Mandushev et al. 2007) and KELT (e.g., Siverd et al. 2012), a total of 45 well-characterized highly inflated planets are now known, allowing us to explore some of their statistical properties. In this paper we find that inflated planets are more commonly found around moderately evolved stars that are more than $50 \%$ of the way through their main-sequence lifetimes. Smaller-radius close-in giant planets, by contrast, are generally found around less evolved stars. Taken at face value, this suggests that planets are reinflated as

\footnotetext{
16 This radius is chosen simply for illustrative purposes and is not meant to imply that planets with radii above this value are physically distinct from those with radii below this value.
}

they age, and indicates that energy must be transferred deep into the planetary interiors (e.g., Liu et al. 2008).

Of course, observational selection effects or systematic errors in the determination of stellar and planetary properties could potentially be responsible for the correlation as well. We therefore consider a variety of potentially important effects, such as the effect of stellar evolution on the detectability of transits and our ability to confirm planets through follow-up observations, and systematic errors in the orbital eccentricity, transit parameters, stellar atmospheric parameters, or the comparison to stellar evolution models. We conclude that the net selection effect would, if anything, tend to favor the discovery of large planets around less evolved stars, while potential systematic errors are too small to explain the correlation. We also show that the correlation remains significant even after accounting for nontrivial truncations placed on the data as a result of the observational selection biases. We are therefore confident in the robustness of this result.

The organization of the paper is as follows. In Section 2 we describe the photometric and spectroscopic observations made to discover and characterize HAT-P-65b and HAT-P-66b. In Section 3 we present the analysis carried out to determine the stellar and planetary parameters and to rule out blended stellar eclipsing binary false-positive scenarios. In Section 4 we place these planets into context and find that large-radius planets are more commonly found around moderately evolved, brighter stars. We provide a brief summary of the results in Section 5.

\section{OBSERVATIONS}

\subsection{Photometric Detection}

Both HAT-P-65 (R.A. $=21^{\mathrm{h}} 03^{\mathrm{m}} 37^{\mathrm{s}} .44, \quad$ decl. $=$ $+11^{\circ} 59^{\prime} 21$ ! $^{\prime} 9$ [J2000], $V=13.145 \pm 0.029 \mathrm{mag}$, spectral type G2) and HAT-P-66 (R.A. $=10^{\mathrm{h}} 02^{\mathrm{m}} 17^{\mathrm{s}} .52$, decl. $=$ $+53^{\circ} 57^{\prime} 03$ ". 1 [J2000], $V=12.993 \pm 0.052 \mathrm{mag}$, spectral type G0) were selected as candidate transiting planet systems based on Sloan $r$-band photometric time series observations carried out with the HATNet telescope network (Bakos et al. 2004).

HATNet consists of six $11 \mathrm{~cm}$ aperture telephoto lenses, each coupled to an APOGEE front-side-illuminated CCD camera, and each placed on a fully automated telescope mount. Four of the instruments are located at Fred Lawrence Whipple Observatory (FLWO) in Arizona, USA, while two are located on the roof of the Submillimeter Array hangar building at Mauna Kea Observatory (MKO) on the island of Hawaii, USA. Each instrument observes a $10^{\circ} .6 \times 10^{\circ} .6$ field of view and continuously monitors one or two fields each night, where a field corresponds to one of 838 fixed pointings used to cover the full $4 \pi$ celestial sphere. A typical field is observed for approximately 3 months using one or two instruments (e.g., field G342 containing HAT-P-65), while a handful of fields have been observed extensively using all six instruments in the network and with observations repeated in multiple seasons (e.g., field G101 containing HAT-P-66). The former observing strategy maximizes the sky coverage of the survey, while maintaining nearly complete sensitivity to transiting giant planets with orbital periods of a few days. The latter strategy substantially increases the sensitivity to Neptune- and superEarth-size planets, as well as planets with periods greater than 10 days, but with the trade-off of covering a smaller area of the sky. 
Table 1

Summary of Photometric Observations

\begin{tabular}{|c|c|c|c|c|c|}
\hline Instrument/Field ${ }^{\mathrm{a}}$ & Date (s) & \# Images & $\begin{array}{c}\text { Cadence }^{\mathrm{b}} \\
\text { (s) }\end{array}$ & Filter & $\begin{array}{c}\text { Precisiono }^{\mathrm{c}} \\
\text { (mmag) }\end{array}$ \\
\hline \multicolumn{6}{|l|}{ HAT-P-65 } \\
\hline HAT-6/G342 & 2009 Sep-Dec & 2738 & 231 & $r$ & 16.7 \\
\hline FLWO 1.2 m/KeplerCam & 2011 Jun 10 & 86 & 124 & $i$ & 1.6 \\
\hline FLWO $1.2 \mathrm{~m} /$ KeplerCam & 2011 Jun 26 & 108 & 135 & $i$ & 1.3 \\
\hline FLWO $1.2 \mathrm{~m} /$ KeplerCam & $2011 \mathrm{Jul} 14$ & 73 & 133 & $i$ & 2.3 \\
\hline FLWO $1.2 \mathrm{~m} /$ KeplerCam & 2013 Sep 16 & 188 & 60 & $z$ & 3.3 \\
\hline FLWO $1.2 \mathrm{~m} /$ KeplerCam & 2013 Sep 29 & 295 & 60 & $i$ & 1.4 \\
\hline FLWO $1.2 \mathrm{~m} /$ KeplerCam & 2013 Oct 04 & 294 & 60 & $i$ & 1.3 \\
\hline \multicolumn{6}{|l|}{ HAT-P-66 } \\
\hline HAT-10/G101 & 2011 Feb-2012 Mar & 2029 & 212 & $r$ & 16.8 \\
\hline HAT-5/G101 & 2011 Feb-2012 Apr & 1520 & 214 & $r$ & 18.1 \\
\hline FLWO $1.2 \mathrm{~m} /$ KeplerCam & 2015 Apr 29 & 204 & 59 & $i$ & 1.9 \\
\hline FLWO $1.2 \mathrm{~m} /$ KeplerCam & 2015 Nov 26 & 273 & 60 & $z$ & 1.9 \\
\hline FLWO $1.2 \mathrm{~m} /$ KeplerCam & 2015 Dec 08 & 131 & 60 & $i$ & 1.9 \\
\hline
\end{tabular}

Notes.

${ }^{\mathrm{a}}$ For HATNet data we list the HATNet unit and field name from which the observations are taken. HAT-5, HAT-6, HAT-7, and HAT-10 are located at Fred Lawrence Whipple Observatory in Arizona. HAT-8 and HAT-9 are located on the roof of the Smithsonian Astrophysical Observatory Submillimeter Array hangar building at Mauna Kea Observatory in Hawaii. Each field corresponds to one of 838 fixed pointings used to cover the full $4 \pi$ celestial sphere. All data from a given HATNet field are reduced together, while detrending through external parameter decorrelation (EPD) is done independently for each unique unit+field combination.

${ }^{\mathrm{b}}$ The median time between consecutive images rounded to the nearest second. Due to factors such as weather, the day-night cycle, guiding, and focus corrections, the cadence is only approximately uniform over short timescales.

${ }^{\mathrm{c}}$ The rms of the residuals from the best-fit model.
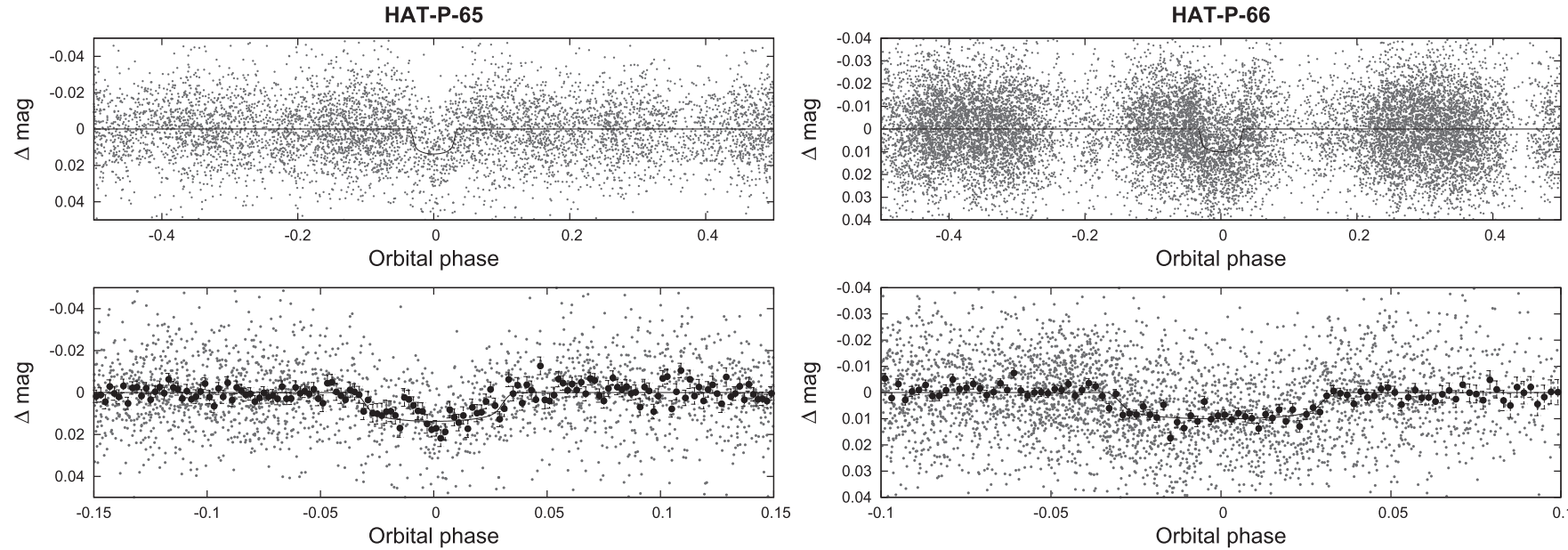

Figure 1. Phase-folded unbinned HATNet light curves for HAT-P-65 (left) and HAT-P-66 (right). In each case we show two panels. The top panel shows the full light curve, while the bottom panel shows the light curve zoomed in on the transit. The solid lines show the model fits to the light curves. The dark filled circles in the bottom panels show the light curves binned in phase with a bin size of 0.002 .

Table 1 summarizes the properties of the HATNet observations collected for each system, including which HAT instruments were used, the date ranges over which each target was observed, the median cadence of the observations, and the per-point photometric precision after trend filtering.

We reduce the HATNet observations to light curves, for all stars in a field with $r<14.5$, following Bakos et al. (2004). We used aperture photometry routines based on the FITSH software package (Pál 2012) and filtered systematic trends from the light curves following Kovács et al. (2005) (i.e., TFA) and Bakos et al. (2010) (i.e., EPD). Transits were identified in the filtered light curves using the Box-fitting Least Squares method (BLS; Kovács et al. 2002). After identifying the transits, we then reapplied TFA while preserving the shape of the transit signal as described in Kovács et al. (2005). This procedure is referred to as signal-reconstruction TFA. The final 
trend-filtered and signal-reconstructed light curves are shown phase-folded in Figure 1, while the measurements are available in Table 2.

We searched the residual HATNet light curves of both objects for additional periodic signals using BLS. Neither target shows evidence for additional transits with BLS; however, this conclusion depends on the set of template light curves used in applying signal-reconstruction TFA to remove systematics. For HAT-P-65 we find that with an alternative set of templates the residuals display a marginally significant transit signal with a period of 2.573 days, which is only slightly different from the main transit period of $2.6054552 \pm 0.0000031$ days. The transits detected at this period come from data points near orbital phase 0.25 when phased at the primary transit period. Since the detection of this additional signal depends on the template set used, and since any planet orbiting with a period so close to (but not equal to) that of the hot Jupiter HAT-P-65b would almost certainly be unstable, we suspect that the $P=2.573$ day transit signal is not of physical origin.

We also searched the residual light curves for periodic signals using the Generalized Lomb-Scargle method (GLS; Zechmeister \& Kürster 2009). For HAT-P-65 no statistically significant signal is detected in the GLS periodogram either. The highest peak in the periodogram is at a period of 0.035 days and has a semiamplitude of $1.2 \mathrm{mmag}$ (using a Markov Chain Monte Carlo procedure to fit a sinusoid with a variable period yields a 95\% confidence upper limit of $1.7 \mathrm{mmag}$ on the semiamplitude). For HAT-P-66, for our default light curve (i.e., the one included in Table 2), we do see significant peaks in the periodogram at periods of $P=83.3029$ and 0.98664 days (and its harmonics) and with formal falsealarm probabilities of $10^{-11}$ and semiamplitudes of $\sim 0.02 \mathrm{mag}$. Given the effective sampling rate of the observations, the two signals are aliases of each other. Based on an inspection of the light curve, we conclude that this detected variability is likely due to additional systematic errors in the photometry that were not effectively removed by our filtering procedures, and that the signal is not astrophysical in nature. Indeed, if we use an alternative TFA template set in filtering the HAT-P-66 light curve, we detect no significant signal in the GLS spectrum, and we place an upper limit on the amplitude of any periodic signal of $1 \mathrm{mmag}$.

\subsection{Spectroscopic Observations}

Spectroscopic observations of both HAT-P-65 and HAT-P66 were carried out using TRES (Füresz 2008) on the $1.5 \mathrm{~m}$ Tillinghast Reflector at FLWO, and HIRES (Vogt et al. 1994) on the Keck I $10 \mathrm{~m}$ at MKO. For HAT-P-65 we also obtained observations using the Fibre-fed Échelle Spectrograph (FIES) on the $2.5 \mathrm{~m}$ Nordic Optical Telescope (NOT; Djupvik \& Andersen 2010) at the Observatorio del Roque de los Muchachos on the Spanish island of La Palma. For HAT-P66 spectroscopic observations were also collected using the SOPHIE spectrograph on the $1.93 \mathrm{~m}$ telescope at the Observatoire de Haute-Provence (OHP; Bouchy et al. 2009) in France. The spectroscopic observations collected for each system are summarized in Table 3. Phase-folded high-precision radial velocity (RV) and spectral line bisector span (BS) measurements are plotted in Figure 2, together with our best-fit models for the RV orbital wobble of the host stars (Section 3.3). The individual RV and BS measurements are made available in Table 4 at the end of the paper.
The TRES observations were reduced to spectra and crosscorrelated against synthetic stellar templates to measure the RVs and to estimate $T_{\text {eff }}, \log g_{\star}$, and $v \sin i$. Here we followed the procedure of Buchhave et al. (2010), initially making use of a single order containing the gravity- and temperature-sensitive $\mathrm{Mg} \mathrm{b}$ lines. Based on these "reconnaissance" observations, we quickly ruled out common false-positive scenarios, such as transiting M dwarf stars, or blends between giant stars and pairs of eclipsing dwarf stars (e.g., Latham et al. 2009). For HAT-P65 we only obtained a single TRES observation, which, in combination with the FIES observations discussed below, rules out these false-positive scenarios. For HAT-P-66 the initial TRES RVs showed evidence of an orbital variation consistent with a planetary-mass companion producing the transits detected by HATNet, so we continued collecting higher signal-to-noise ratio $(\mathrm{S} / \mathrm{N})$ observations of this system with TRES. High-precision RVs and BSs were measured from these spectra via a multi-order analysis (e.g., Bieryla et al. 2014).

The FIES spectra of HAT-P-65 were reduced in a similar manner to the TRES data (Buchhave et al. 2010) and were used for reconnaissance. Two exposures were obtained using the medium-resolution fiber, while the third was obtained with the high-resolution fiber. One of the two medium-resolution observations had sufficiently high $\mathrm{S} / \mathrm{N}$ to be used for characterizing the stellar atmospheric parameters (Section 3.1).

The HIRES observations of HAT-P-65 and HAT-P-66 were reduced to relative RVs in the solar system barycenter frame following the method of Butler et al. (1996), and to BSs following Torres et al. (2007). We also measured Ca II HK chromospheric emission indices (the so-called $S$ and $\log _{10} R_{\mathrm{HK}}^{\prime}$ indices) following Isaacson \& Fischer (2010) and Noyes et al. (1984). The $I_{2}$-free template observations of each system were also used to determine the adopted stellar atmospheric parameters (Section 3.1).

The SOPHIE spectra of HAT-P-66 were collected as described in Boisse et al. (2013) and reduced following Santerne et al. (2014). One of the observations was obtained during a planetary transit and is excluded from the analysis.

\subsection{Photometric Follow-up Observations}

In order to better determine the physical parameters of each TEP system and to aid in excluding blended stellar eclipsing binary false-positive scenarios, we conducted follow-up photometric time series observations of each object using KeplerCam on the $1.2 \mathrm{~m}$ telescope at FLWO. These observations are summarized in Table 1, where we list the dates of the observed transit events, the number of images collected for each event, the cadence of the observations, the filters used, and the per-point photometric precision achieved after trend filtering. The images were reduced to light curves via aperture photometry based on the FITSH package (following Bakos et al. 2010) and filtered for trends, which were fit to the light curves simultaneously with the transit model (Section 3.3). The resulting trend-filtered light curves are plotted together with the best-fit transit model in Figure 3 for HAT-P-65 and in Figure 4 for HAT-P-66. The data are made available in Table 2.

\subsection{Imaging Constraints on Resolved Neighbors}

In order to detect possible neighboring stars that may be diluting the transit signals, we obtained $J$ - and $K_{S}$-band snapshot images of both targets using the WIYN High- 
Table 2

Light-curve Data for HAT-P-65 and HAT-P-66

\begin{tabular}{|c|c|c|c|c|c|c|}
\hline$\overline{\text { Object }^{\mathrm{a}}}$ & $\begin{array}{c}\text { BJD }^{\mathrm{b}} \\
(2,400,000+)\end{array}$ & $\mathrm{Mag}^{\mathrm{c}}$ & $\sigma_{\mathrm{Mag}}$ & Mag(orig) ${ }^{\mathrm{d}}$ & Filter & $\overline{\text { Instrument }}$ \\
\hline HAT-P-65 & 55128.75175 & -0.00921 & 0.01482 & $\cdots$ & $r$ & HATNet \\
\hline HAT-P-65 & 55115.72468 & -0.00946 & 0.01149 & $\cdots$ & $r$ & HATNet \\
\hline HAT-P-65 & 55115.72489 & -0.01377 & 0.01518 & $\ldots$ & $r$ & HATNet \\
\hline HAT-P-65 & 55094.88211 & -0.01227 & 0.01156 & $\ldots$ & $r$ & HATNet \\
\hline HAT-P-65 & 55128.75370 & -0.00283 & 0.01249 & $\cdots$ & $r$ & HATNet \\
\hline HAT-P-65 & 55128.75445 & -0.00558 & 0.01427 & $\ldots$ & $r$ & HATNet \\
\hline HAT-P-65 & 55115.72734 & 0.00413 & 0.01208 & $\ldots$ & $r$ & HATNet \\
\hline
\end{tabular}

Notes.

${ }^{\mathrm{a}}$ Either HAT-P-65 or HAT-P-66.

b Barycentric Julian Date is computed directly from the UTC time without correction for leap seconds.

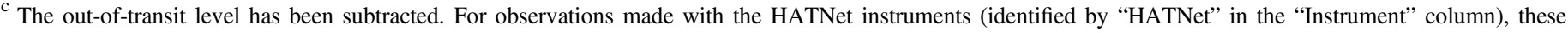

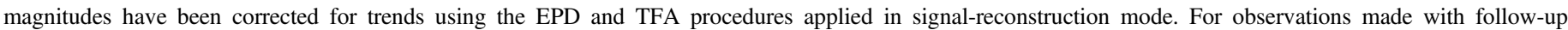

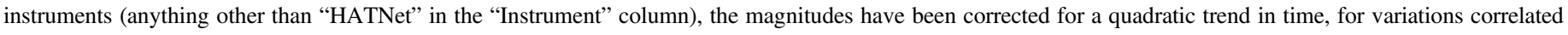

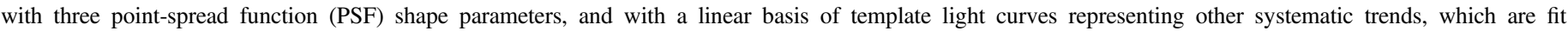
simultaneously with the transit.

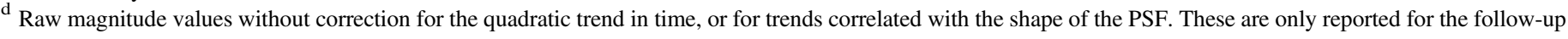
observations.

(This table is available in its entirety in machine-readable form.)

Resolution Infrared Camera (WHIRC) on the WIYN $3.5 \mathrm{~m}$ telescope at Kitt Peak National Observatory in Arizona. Observations were obtained on the nights of 2016 April 24, 27 , and 28 , with seeing varying between $\sim 00^{\prime \prime} 5$ and $\sim 1^{\prime \prime}$. Images were collected at different nod positions. These were calibrated, background-subtracted, registered, and mediancombined using the same tools that we used for reducing the KeplerCam images.

We find that HAT-P-65 has a neighbor located 3"' 6 to the west with a magnitude difference of $\Delta J=4.91 \pm 0.01 \mathrm{mag}$ and $\Delta K=4.95 \pm 0.03$ mag relative to HAT-P-65 (Figure 5). The neighbor is too faint and distant to be responsible for the transits detected in either the HATNet or KeplerCam observations. The neighbor has a $J-K$ color that is the same as HATP-65 to within the uncertainties, and is thus a background star with an effective temperature that is similar to that of HAT-P65 , and not a physical companion. No neighbor is detected within $10^{\prime \prime}$ of HAT-P-66.

Figure 6 shows the $J$ - and $K$-band magnitude contrast curves for HAT-P-65 and HAT-P-66 based on these observations. These curves are calculated using the method and software described by Espinoza et al. (2016). The bands shown in these images represent the variation in the contrast limits depending on the position angle of the putative neighbor.

\section{ANALYSIS}

\subsection{Properties of the Parent Star}

High-precision atmospheric parameters, including the effective surface temperature $T_{\text {eff } \star}$, the surface gravity $\log g_{\star}$, the metallicity $[\mathrm{Fe} / \mathrm{H}]$, and the projected rotational velocity $v \sin i$, were determined by applying the Stellar Parameter Classification (SPC; Buchhave et al. 2012) procedure to our highresolution spectra. For HAT-P-65 this analysis was performed on the highest-S/N FIES spectrum and on our Keck I/HIRES
$\mathrm{I}_{2}$-free template spectrum (we adopt the weighted average of each parameter determined from the two spectra). For HAT-P66 this analysis was performed on our Keck I/HIRES I ${ }_{2}$-free template spectrum. We assume a minimum uncertainty of $50 \mathrm{~K}$ on $T_{\text {eff }}, 0.10$ dex on $\log g_{\star}, 0.08$ dex on $[\mathrm{Fe} / \mathrm{H}]$, and $0.5 \mathrm{~km} \mathrm{~s}^{-1}$ on $v \sin i$, which reflects the systematic uncertainty in the method and is based on applying the SPC analysis to observations of spectroscopic standard stars.

Following Sozzetti et al. (2007), we combine the $T_{\text {eff } \star}$ and $[\mathrm{Fe} / \mathrm{H}]$ values measured from the spectra with the stellar densities $\left(\rho_{\star}\right)$ determined from the light curves (based on the analysis in Section 3.3) to determine the physical parameters of the host stars (i.e., their masses, radii, surface gravities, ages, luminosities, and broadband absolute magnitudes) via interpolation within the Yonsei-Yale theoretical stellar isochrones (YY; Yi et al. 2001). Figure 7 compares the model isochrones to the measured $T_{\text {eff } \star}$ and $\rho_{\star}$ values for each system.

For HAT-P-65 the $\log g_{\star}$ value determined from this analysis differed by $0.19 \mathrm{dex}(\sim 1.9 \sigma)$ from the initial value determined through SPC. A difference of this magnitude is typical and reflects the difficulty of accurately measuring all four atmospheric parameters simultaneously via cross-correlation with synthetic templates (e.g., Torres et al. 2012). We therefore carried out a second SPC analysis of HAT-P-65 with $\log g_{\star}$ fixed based on this analysis, and then repeated the light-curve analysis and stellar parameter determination, finding no appreciable change in $\log g_{\star}$. For HAT-P-66 the $\log g_{\star}$ value determined from the YY isochrones differed by only 0.007 dex from the initial spectroscopically determined value, so we did not carry out a second SPC iteration in this case.

The adopted stellar parameters for HAT-P-65 and HAT-P-66 are listed in Table 5. We also collect in this table a variety of photometric and kinematic properties for each system from catalogs. Distances are determined using the listed photometry and assuming an $R_{V}=3.1$ Cardelli et al. (1989) extinction law. 

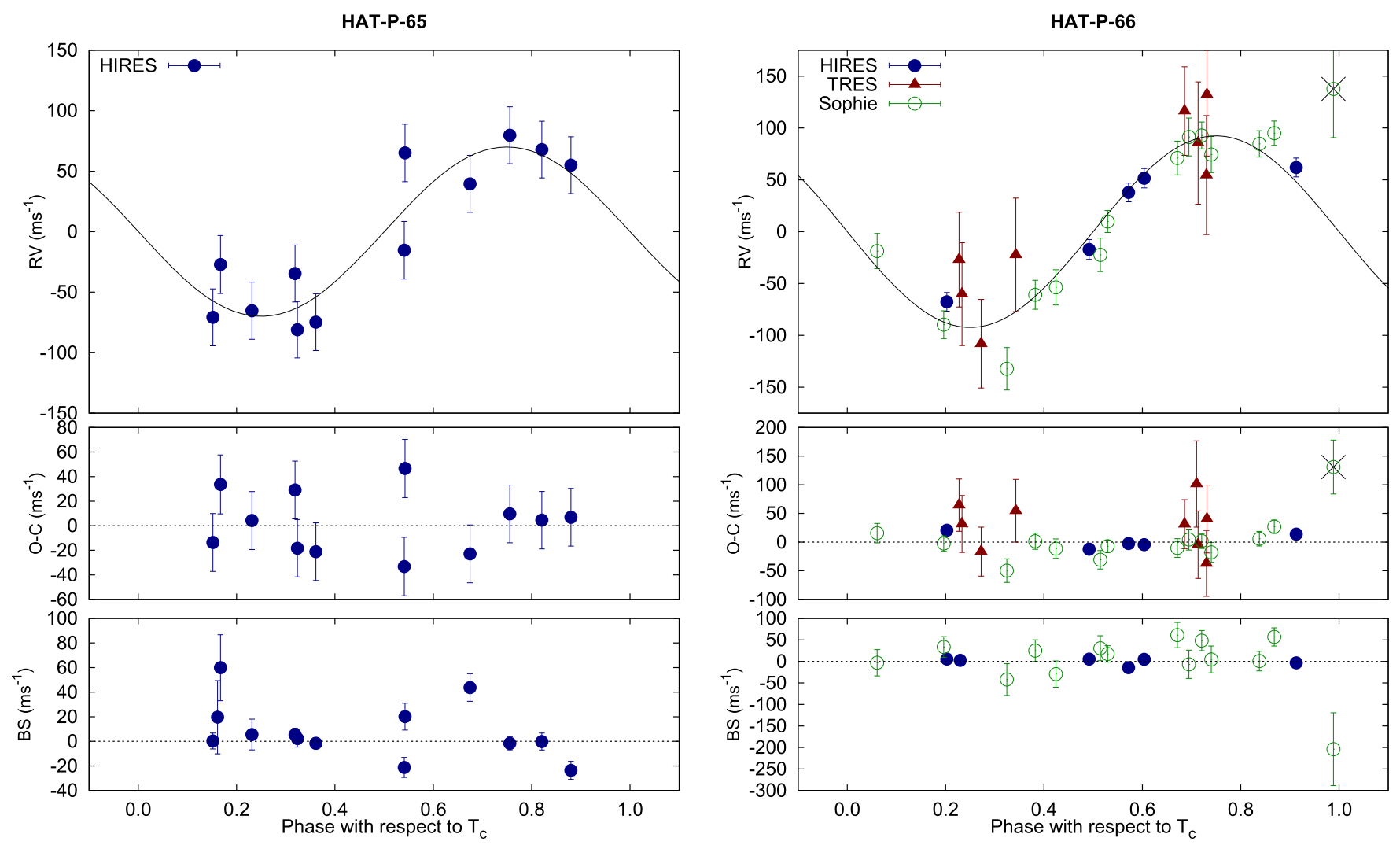

Figure 2. Phase-folded high-precision RV measurements for HAT-P-65 and HAT-P-66. The instruments used are labeled in the plots. In each case we show three panels. The top panel shows the phased measurements, together with our best-fit circular-orbit model (see Table 6) for each system. Zero phase corresponds to the time of mid-transit. The center-of-mass velocity has been subtracted. The second panel shows the velocity $O-C$ residuals from the best fit. The error bars include the jitter terms listed in Table 6 added in quadrature to the formal errors for each instrument. The third panel shows the bisector spans (BS). Note the different vertical scales of the panels. For HAT-P-66 the crossed-out SOPHIE measurement was obtained during transit and is excluded from the analysis.

The two stars are quite similar, with masses of $1.212 \pm 0.050 M_{\odot}$ and $1.255_{-0.054}^{+0.107} M_{\odot}$ for HAT-P-65 and HAT-P-66, respectively, and with respective radii of $1.860 \pm 0.096 R_{\odot}$ and $1.881_{-0.095}^{+0.151} R_{\odot}$. The stars are moderately evolved, with ages of $5.46 \pm 0.61 \mathrm{Gyr}$ and $4.66_{-1.12}^{+0.52} \mathrm{Gyr}$ (these are $84 \% \pm 10 \%$ and $83_{-20}^{+9} \%$ of each star's full lifetime, respectively). As we point out in Section 4, there appears to be a general trend among the host stars of highly inflated planets in which the largest planets are preferentially found around moderately evolved stars. HAT-P-65 and HAT-P-66 are in line with this trend.

\subsection{Excluding Blend Scenarios}

In order to exclude blend scenarios, we carried out an analysis following Hartman et al. (2012). Here we attempt to model the available photometric data (including light curves and catalog broadband photometric measurements) for each object as a blend between an eclipsing binary star system and a third star along the line of sight (either a physical association or a chance alignment). The physical properties of the stars are constrained using the Padova isochrones (Girardi et al. 2002), while we also require that the brightest of the three stars in the blend have atmospheric parameters consistent with those measured with SPC. We also simulate composite crosscorrelation functions (CCFs) and use them to predict RVs and BSs for each blend scenario considered.

Based on this analysis, we rule out blended stellar eclipsing binary scenarios for both HAT-P-65 and HAT-P-66. For HAT-
P-65 we are able to exclude blend scenarios, based solely on the photometry, with greater than $3.7 \sigma$ confidence, while for HAT-P-66 we are able to exclude them with greater than $3.9 \sigma$ confidence. For both objects, the blend models that come closest to fitting the photometric data (those that could not be rejected with $5 \sigma$ confidence) can additionally be rejected due to the predicted large-amplitude BS and RV variations, which we do not observe.

\subsection{Global Modeling of the Data}

In order to determine the physical parameters of the TEP systems, we carried out a global modeling of the HATNet and KeplerCam photometry and the high-precision RV measurements following Pál et al. (2008), Bakos et al. (2010), and Hartman et al. (2012). We use the Mandel \& Agol (2002) transit model to fit the light curves, with limb-darkening coefficients fixed to the values tabulated by Claret (2004) for the atmospheric parameters of the stars and the broadband filters used in the observations. For the KeplerCam follow-up light curves we account for instrumental variations by using a set of linear basis vectors in the fit. The vectors that we use include the time of observations, the time squared, three parameters describing the shape of the PSF, and light curves for the 20 brightest nonvariable stars in the field (TFA templates). For the TFA templates we use the same linear coefficient (which is varied in the fit) for all light curves collected for a given transiting planet system through a given filter, while for the other basis vectors we use a different coefficient for each 


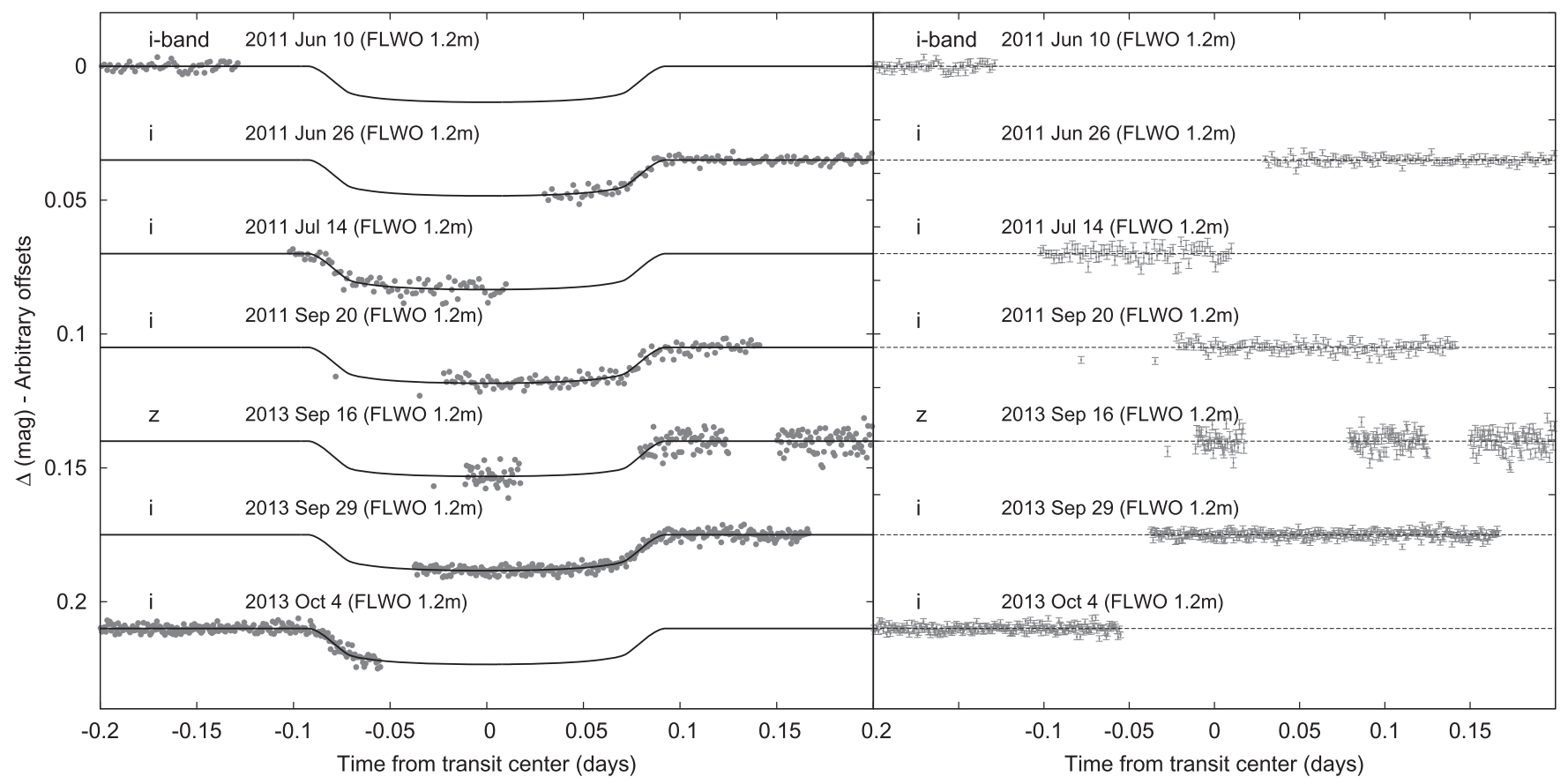

Figure 3. Left: unbinned transit light curves for HAT-P-65. The light curves have been filtered of systematic trends, which were fit simultaneously with the transit model. The dates of the events, filters, and instruments used are indicated. Light curves following the first are displaced vertically for clarity. Our best fit from the global modeling described in Section 3.3 is shown by the solid lines. Right: residuals from the best-fit model are shown in the same order as the original light curves. The error bars represent the photon and background shot noise, plus the readout noise.

light curve. For the HATNet light curves we use a Mandel \& Agol (2002) model and apply the fit to the signal-reconstruction TFA data (see Section 2.1). The RV curves are modeled using a Keplerian orbit, where we allow the zero point for each instrument to vary independently in the fit, and we include an $\mathrm{RV}$ jitter term added in quadrature to the formal uncertainties. The jitter is treated as a free parameter, which we fit for, and is taken to be independent for each instrument.

All observations of an individual system are modeled simultaneously using a Differential Evolution Markov Chain Monte Carlo procedure (ter Braak 2006). We visually inspect the Markov Chains and also apply a Geweke (1992, pp. 169-93) test to verify convergence and determine the burn-in period. For both systems we consider two models: a fixedcircular-orbit model and an eccentric-orbit model. To determine which model to use, we estimate the Bayesian evidence ratio from the Markov Chains following Weinberg et al. (2013) and find that for both systems the fixed-circular model has a greater evidence, and therefore we adopt the parameters that come from this model. The resulting parameters for both planetary systems are listed in Table 6 . We also list the $95 \%$ confidence upper limit on the eccentricity for each system.

We find that HAT-P-65b has a mass of $0.527 \pm 0.083 M_{\mathrm{J}}$, a radius of $1.89 \pm 0.13 R_{\mathrm{J}}$, and an equilibrium temperature (assuming zero albedo and full redistribution of heat) of $1930 \pm 45 \mathrm{~K}$ and is consistent with a circular orbit, with a 95\% confidence upper limit on the eccentricity of $e<0.304$. HAT$\mathrm{P}-66 \mathrm{~b}$ has similar properties, with a mass of $0.783 \pm 0.057 M_{\mathrm{J}}$, a radius of $1.59_{-0.10}^{+0.16} R_{\mathrm{J}}$, an equilibrium temperature (same assumptions) of $1896_{-42}^{+66} \mathrm{~K}$, and an eccentricity of $e<0.090$ with $95 \%$ confidence.

\section{DISCUSSION}

\subsection{Large-radius Planets More Commonly Found around More Evolved Stars}

With radii of $1.89 \pm 0.13 R_{\mathrm{J}}$ and $1.59_{-0.10}^{+0.16} R_{\mathrm{J}}$, HAT-P-65b and HAT-P-66b are among the largest hot Jupiters known. Both planets are found around moderately evolved stars approaching the end of their main-sequence lifetimes. With an estimated age of $5.46 \pm 0.61 \mathrm{Gyr}$, HAT-P-65 is $84 \%$ $\pm 10 \%$ of the way through its total life span, while HAT-P66 , with an age of $4.66_{-1.12}^{+0.52} \mathrm{Gyr}$, is $83_{-20}^{+9} \%$ of the way through its life span. Looking at the broader sample of TEPs that have been discovered to date, we find that the largest exoplanets are preferentially found around moderately evolved stars.

This effect may be a by-product of the more physically important correlation between planet radius and equilibrium temperature (e.g., Fortney et al. 2007; Enoch et al. 2012; and Spiegel \& Burrows 2013), with the planet equilibrium temperature increasing in time as its host star evolves and becomes more luminous. We will address the question of how the correlation between planet radius and host star fractional age that we demonstrate here relates to the radius-equilibrium temperature correlation in Section 4.1.1. While the planet radius-equilibrium temperature correlation is well known, whether or not the radii of planets can actually increase in time as their equilibrium temperatures increase has not been previously established. As discussed in Sections 1 and 4.1.4, the answer to this question has important theoretical implications for understanding the physical mechanism behind the inflation of close-in giant planets. To address this question, we will first attempt to determine whether or not there is a statistically signification correlation between planetary radii and the evolutionary status of their host stars. 


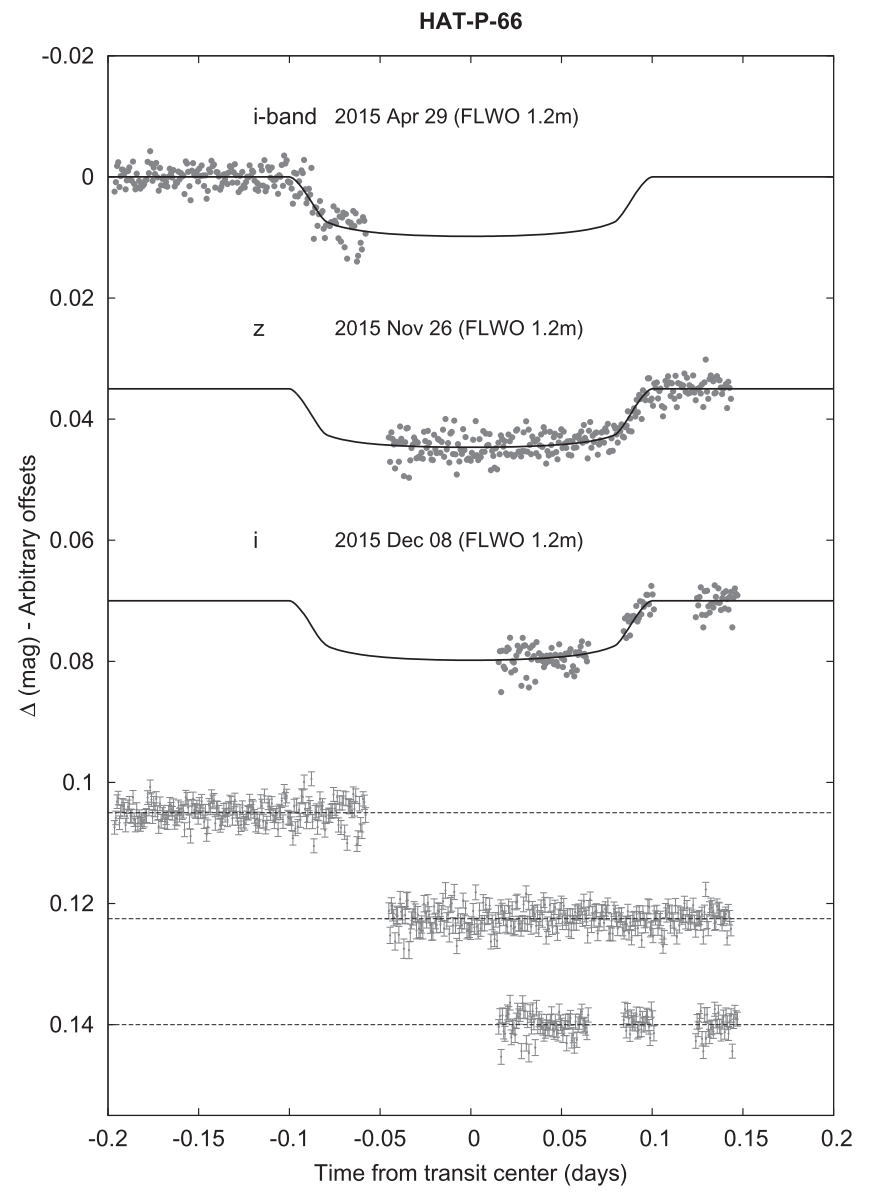

Figure 4. Similar to Figure 3, but here we show unbinned transit light curves for HAT-P-66. The residuals in this case are shown below in the same order as the original light curves.

Figure 8 shows TEP host stars on a $T_{\text {eff } \star}-\rho_{\star}$ diagram. These two parameters are directly measured for TEP systems and, together with the $[\mathrm{Fe} / \mathrm{H}]$ of the star, are the primary parameters used to characterize the stellar hosts. Here we limit the sample to systems with planets having $R_{p}>0.5 R_{\mathrm{J}}$ and $P<10$ days. Because observational selection effects vary from survey to survey, we show separately the systems discovered by HAT (both HATNet and HATSouth), WASP, Kepler, TrES, and KELT, which are the surveys that have discovered wellcharacterized planets with $R_{p}>1.5 R_{\mathrm{J}}$. The data for the HAT, WASP, TrES, and KELT systems are drawn from a database of TEPs that we privately maintain, and are listed, together with references, in Table 7 at the end of this paper. These are planets that have been announced on the arXiv pre-print server as of 2016 June 2, and are supplemented by some additional fully confirmed planets from HAT that had not been announced by that date. For Kepler we take the data from the NASA Exoplanet archive. ${ }^{17}$ In Figure 8 we distinguish between hosts with planets having $R_{p}>1.5 R_{\mathrm{J}}$ and hosts with planets having $R_{p}<1.5 R_{\mathrm{J}}$. The lower bound in each panel shows the solarmetallicity, 200 Myr ZAMS isochrone from the YY models, while the upper bound shows the locus of points for stars having an age that is the lesser of 13.7 Gyr or $90 \%$ of their total lifetime, again assuming solar metallicity and using the YY models. For all of the surveys considered, planets with $R>1.5$

\footnotetext{
${ }^{17}$ http://exoplanetarchive.ipac.caltech.edu, accessed 2016 March 4.
}

$R_{\mathrm{J}}$ tend to be found around host stars that are more evolved (closer to the $90 \%$ lifetime locus) than planets with $R<1.5 R_{\mathrm{J}}$. Moreover, very few highly inflated planets have been discovered around stars close to the ZAMS. The largest planets also tend to be found around hot/massive stars and have the highest level of irradiation.

For another view of the data, in Figure 9 we plot the massradius relation of close-in TEPs with the color scale of each point showing the fractional isochrone-based age of the system (taken to be equal to $\left.\tau=(t-200 \mathrm{Myr}) /\left(t_{\mathrm{tot}}-200 \mathrm{Myr}\right)\right)$. Here $t_{\text {tot }}$ for a system is the maximum age of a star with a given mass and metallicity according to the YY models (Figure 10). We show the fractional age, rather than the age in Gyr, as the stellar lifetime is a strong function of stellar mass, and the largest planets also tend to be found around more massive stars with shorter total lifetimes. Because the star formation rate in the Galaxy has been approximately constant over the past $\sim 8$ Gyr (e.g., Snaith et al. 2015), for a star of a given mass we expect $\tau$ to be uniformly distributed between 0 and 1 . In order to perform a consistent analysis, we recompute ages for all of the WASP, Kepler, TrES, and KELT systems using the YY models, together with the spectroscopically measured $T_{\text {eff }}$ and $[\mathrm{Fe} / \mathrm{H}]$ and transit-inferred stellar densities listed in Table 7. In Figure 9 we focus on systems with $P<10$ days and $t_{\text {tot }}<10 \mathrm{Gyr}$. Again it is apparent that the largest-radius planets tend to be around stars that are relatively old. Note that due to the finite age of the Galaxy, there has been insufficient time for stars with $t_{\text {tot }}>10 \mathrm{Gyr}$ to reach their main-sequence lifetimes. The restriction on $t_{\text {tot }}$, which is effectively a cut on host star mass, limits the sample to stars that could be discovered at any stage in their evolution. If we do not apply this cut, then the apparent correlation between fractional age and planet radius becomes even more significant, but this is likely due to observational bias.

The planets shown in Figure 9 have a variety of orbital separations and host star masses. Because the evolution of a planet depends on its stellar environment, we expect there to be a variance in the planet radius at fixed planet mass. In order to better compare planets likely to have similar histories (but which have different ages and thus are at different stages in their history), in Figure 11 we replot the mass-radius relation, but this time binning by host star mass and orbital semimajor axis. Note that in comparing planets with the same semimajor axis we are assuming that orbital evolution can be neglected. Again we use the color scale of points to denote the fractional age of the system. We choose a $3 \times 3$ binning to allow a sufficient number of planets in at least some of the bins to be able to detect a statistical trend. Unfortunately, because we limited by the small sample of planets, we are forced to use relatively large bins, so there is likely to still be significant variation in the evolution of different planets within a bin. Bearing this caveat in mind, we note that the same trend of larger-radius planets, at a given planet mass, being found around more evolved stars is seen when comparing only planets with similar host star masses and at similar orbital separations. If anything, the gradient in fractional age with planet radius is more pronounced in Figure 11 than it is in Figure 9 (see especially the center row and bottom center panel of Figure 11). If enhanced irradiation acts to slow a planet's contraction but does not reinflate the planet, then we would expect to see the opposite trend in Figure 11. Namely, a planet of a given mass at a given orbital separation around a star of a 
Table 3

Summary of Spectroscopy Observations

\begin{tabular}{|c|c|c|c|c|c|c|}
\hline Instrument & UT Date (s) & \# Spec. & $\begin{array}{c}\text { Res. } \\
(\lambda / \Delta \lambda) / 1000\end{array}$ & $\mathrm{~S} / \mathrm{N}$ Range $^{\mathrm{a}}$ & $\begin{array}{c}\gamma_{\mathrm{RV}}^{\mathrm{b}} \\
\left(\mathrm{km} \mathrm{s}^{-1}\right)\end{array}$ & $\begin{array}{l}\text { RV Precision }{ }^{\mathrm{c}} \\
\left(\mathrm{m} \mathrm{s}^{-1}\right)\end{array}$ \\
\hline \multicolumn{7}{|l|}{ HAT-P-65 } \\
\hline NOT $2.5 \mathrm{~m} /$ FIES & 2010 Aug 21-22 & 2 & 46 & $24-28$ & -48.131 & 100 \\
\hline FLWO $1.5 \mathrm{~m} /$ TRES & 2010 Oct 27 & 1 & 44 & 16.5 & -47.768 & 100 \\
\hline NOT $2.5 \mathrm{~m} /$ FIES & 2011 Oct 8 & 1 & 67 & 15 & -47.799 & 1000 \\
\hline Keck I/HIRES & 2010 Dec 14 & 1 & 55 & 80 & $\ldots$ & $\ldots$ \\
\hline Keck I/HIRES $+\mathrm{I}_{2}$ & 2010 Dec-2013 Aug & 12 & 55 & $64-106$ & $\cdots$ & 25 \\
\hline \multicolumn{7}{|l|}{$H A T-P-66$} \\
\hline FLWO $1.5 \mathrm{~m} / \mathrm{TRES}$ & 2014 Nov-2015 Jun & 10 & 44 & $17-22$ & 7.973 & 43 \\
\hline OHP $1.93 \mathrm{~m} / \mathrm{SOPHIE}$ & 2015 Mar-2016 Jan & 14 & 39 & $12-33$ & 7.226 & 20 \\
\hline Keck I/HIRES $+\mathrm{I}_{2}$ & 2015 Dec-2016 Jan & 5 & 55 & $78-119$ & $\cdots$ & 12 \\
\hline Keck I/HIRES & 2016 Feb 3 & 1 & 55 & 148 & $\cdots$ & $\cdots$ \\
\hline
\end{tabular}

Notes.

${ }^{a} \mathrm{~S} / \mathrm{N}$ per resolution element near $5180 \AA$ A.

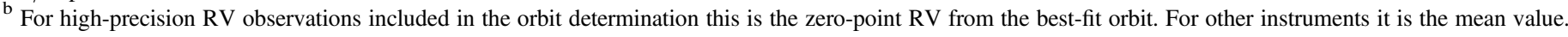
We do not provide this information for Keck I/HIRES, for which only relative velocities are measured.

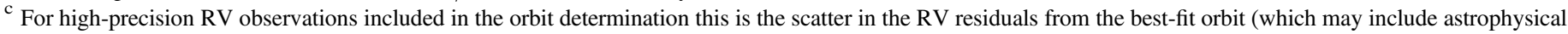

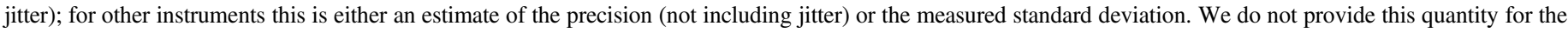
$\mathrm{I}_{2}$-free templates obtained with Keck I/HIRES.

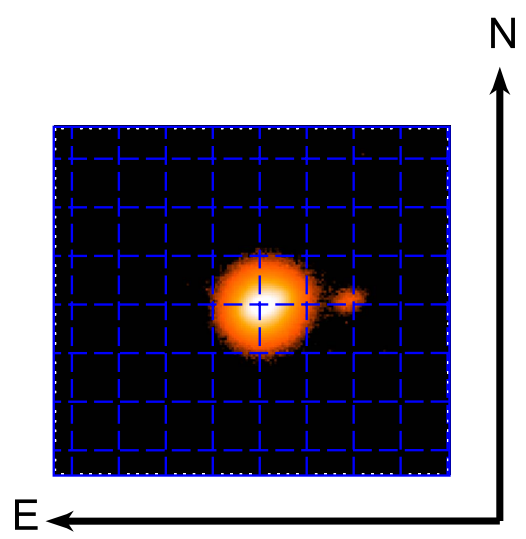

Figure 5. $J$-band image of HAT-P-65 from WHIRC on the WIYN $3.5 \mathrm{~m}$ showing the $\Delta J=4.91 \pm 0.01$ mag neighbor located 3." 6 to the west. The grid spacing is $2^{\prime \prime}$.

given mass should decrease, or remain constant, in size over time, despite the increasing irradiation as its host star evolves. This is not what we see. In Section 4.1.1 we follow a more statistically rigorous method to show that planetary radii increase in time with increasing irradiation, rather than being set by the initial irradiation.

To establish the statistical significance of the trends seen in Figures 8-11, in Figure 12 we plot the fractional isochronebased age $\tau$ against planetary radius, restricted to systems with $t_{\text {tot }}<10$ Gyr. Both the HAT and WASP data have positive correlations between $R_{P}$ and the fractional age. A Spearman nonparametric rank-order correlation test gives a correlation coefficient of 0.344 between $R_{P}$ and the fractional age for HAT, with a $1.4 \%$ false-alarm probability. For the WASP sample we find a correlation coefficient of 0.277 and a false-alarm probability of $3.5 \%$. The Kepler, TrES, and KELT data sets are too small to perform a robust test for correlation, but they each show a similar trend. When all of the data are combined, we find a correlation coefficient of 0.347 and a false-alarm probability of only $0.0041 \%$. While the correlation is relatively weak, explaining only a modest amount of the overall scatter in the data, it has a high statistical significance and is extremely unlikely to be due to random chance.

Figure 13 is similar to Figure 12, except that here we restrict the analysis to planets with $0.4 M_{\mathrm{J}}<M_{p}<2.0 M_{\mathrm{J}}$, which is roughly the range over which the most highly inflated planets have been discovered (e.g., Figure 9). In this case we still find a statistically significant difference between the fractional ages of stars hosting large-radius planets and those hosting smallradius planets, although, due to the smaller sample size, the overall significance is somewhat reduced compared to the sample when no restriction is placed on planet mass (the correlation coefficient itself is somewhat higher). Quantitatively we find that the HAT sample has a Spearman correlation coefficient of 0.428 and a false-alarm probability of $0.84 \%$, the WASP sample has a correlation coefficient of 0.273 and a falsealarm probability of $7.7 \%$, and the combined sample has a correlation coefficient of 0.398 and a false-alarm probability of $0.0068 \%$.

In order to compare planets with similar evolutionary histories, and in analogy to Figure 11, in Figure 14 we plot the fractional age against planet radius gridded by host star mass and orbital semimajor axis. Here we combine all of the data but restrict the sample to only planets with $0.4 M_{\mathrm{J}}<M_{P}<2.0 M_{\mathrm{J}}$ around stars with $t_{\text {tot }}<10 \mathrm{Gyr}$. We see the correlation again in several grid cells, so long as there is a sufficiently large sample.

Of course, these correlations are likely biased due to observational selection effects. We estimate the effect of observational selections on the measured correlation below in Section 4.1.2, where we conclude that the correlation is reduced, but still significant, after accounting for selections.

We conclude that there is a statistically significant positive correlation between $R_{p}$ and the fractional age of the system. 

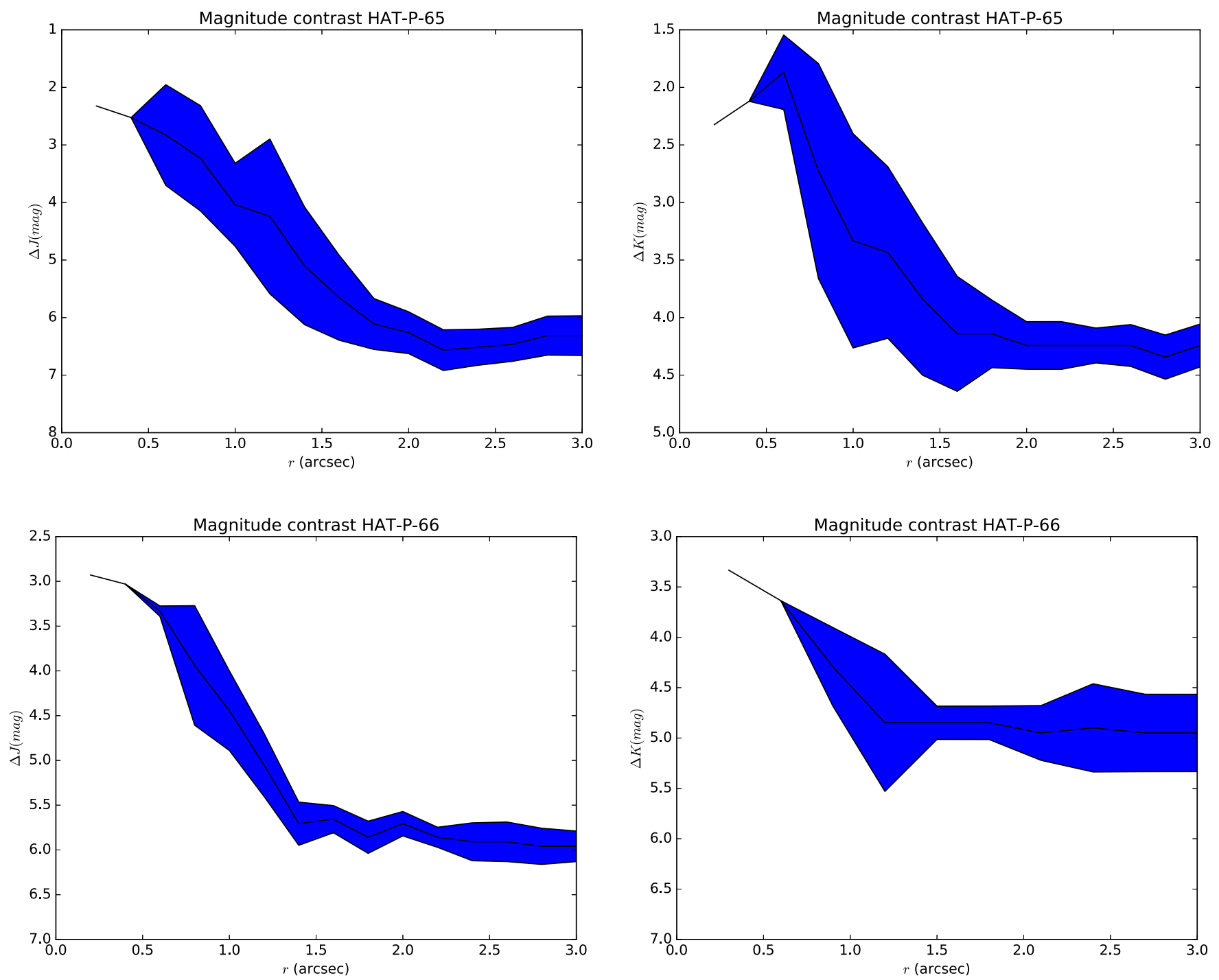

Figure 6. Contrast curves for HAT-P-65 (top) and HAT-P-66 (bottom) in the $J$ band (left) and $K$ band (right) based on observations made with WHIRC on the WIYN $3.5 \mathrm{~m}$ as described in Section 2.4. The bands show the variation in the contrast limits depending on the position angle of the putative neighbor.

This correlation is seen in samples of transiting planets found by multiple surveys, with strikingly similar results found for the largest two samples (from WASP and HAT). The largestradius planets have generally been found around more evolved stars.

\subsubsection{Relation to the Correlation between Radius and Equilibrium Temperature}

It is important to note that the correlation between radius and equilibrium temperature (or flux) is much stronger than the apparent correlation between planet radius and the fractional age of the host star. In fact, the data are consistent with the latter correlation being entirely a by-product of the former correlation. However, the data also indicate that the radii of planets dynamically increase in time as their host stars become more luminous and the planetary equilibrium temperatures increase.

To demonstrate this, we perform a Bayesian linear regression model comparison using the BayesFactor package in $R,{ }^{18}$

\footnotetext{
18 http://bayesfactorpcl.r-forge.r-project.org/
}

which follows the approach of Liang et al. (2008) and Rouder $\&$ Morey (2012). We test models of the form

$$
\ln R_{p}=c_{0}+c_{1} \ln T_{\text {eq,now }}+c_{2} \ln T_{\text {eq,ZAMS }}+c_{3} \ln a+c_{4} \tau,
$$

where $c_{0}-c_{4}$ are varied linear parameters, and we compare all combinations of models where parameters other than $c_{0}$ are fixed to 0 . This particular parameterization is motivated by Enoch et al. (2012), who found that the radii of close-in Jupitermass planets are best modeled by a function of the form given above with $c_{2} \equiv c_{4} \equiv 0$, and we now include the $T_{\text {eq,ZAMS }}$ and $\tau$ parameters to test whether age is an important additional variable, and/or whether the data could be equally well described if we used the initial equilibrium temperature of the planet (which does not change in time) rather than the presentday equilibrium temperature (which increases in time due to the evolution of the host). Here we consider the full sample of well-characterized planets with $P<10$ days, $R_{p}>0.5 R_{\mathrm{J}}$, $0.4 M_{\mathrm{J}}<M_{p}<2.0 M_{\mathrm{J}}$, and $t_{\text {tot }}<10 \mathrm{Gyr}$. Table 8 lists the linear coefficient estimates and the Bayesian evidences, sorting 

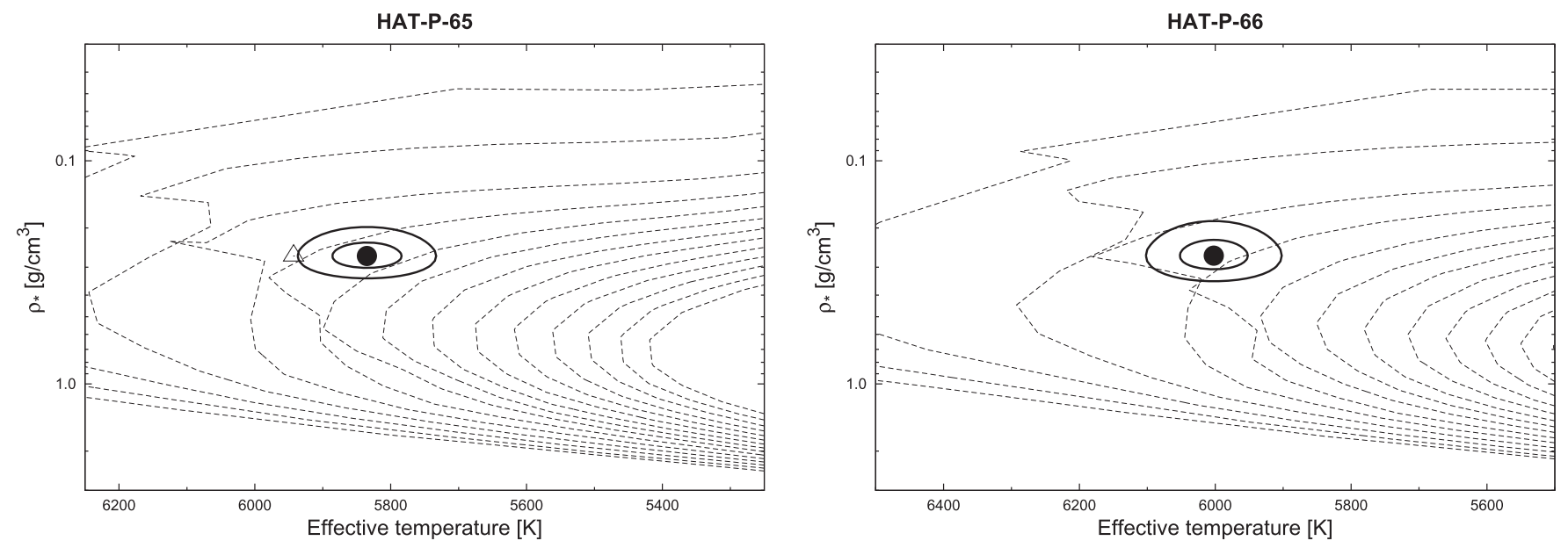

Figure 7. Model isochrones from Yi et al. (2001) for the measured metallicities of HAT-P-65 and HAT-P-66. We show models for ages of 0.2 Gyr and 1.0-14.0 Gyr in $1.0 \mathrm{Gyr}$ increments (ages increasing from left to right). The adopted values of $T_{\text {eff } \star}$ and $\rho_{\star}$ are shown together with their $1 \sigma$ and $2 \sigma$ confidence ellipsoids. The initial values of $T_{\text {eff } \star}$ and $\rho_{\star}$ for HAT-P-65 from the first SPC and light-curve analyses are represented with a triangle.

from highest to lowest, for each of the 15 models under comparison.

We find that the model with the highest Bayesian evidence has the form

$$
\ln R_{p}=c_{0}+c_{1} \ln T_{\text {eq,now }}+c_{3} \ln a
$$

with an evidence that is $2.7 \times 10^{9}$ times higher than the evidence for a model where $c_{1} \equiv c_{2} \equiv c_{3} \equiv c_{4} \equiv 0$. This finding is consistent with that of Enoch et al. (2012). The nexthighest-evidence model has the form

$$
\ln R_{p}=c_{0}+c_{1} \ln T_{\text {eq,now }}+c_{3} \ln a+c_{4} \tau
$$

with an evidence that is 0.37 times that of the highest-evidence model. So indeed including $\tau$ provides no additional explanatory power beyond what is already provided by $T_{\text {eq,now }}$ and $a$.

At the same time, we also find that models with $\ln T_{\text {eq,now }}$ have substantially higher evidence than models using $\ln T_{\text {eq,ZAMs }}$ in place of $\ln T_{\text {eq,now }}$, while the model using both $\ln T_{\text {eq,ZAMS }}$ and $\tau$ has higher evidence than the model using $\ln T_{\text {eq,ZAMS }}$ alone. Moreover, we find that the maximum posterior value for $c_{4}$ is greater than zero in all cases where it is allowed to vary. In other words, planet radii are more strongly correlated with the present-day equilibrium temperature than they are with the zero-age main-sequence (ZAMS) equilibrium temperature, and if the latter is used in place of the former, then a significant positive correlation between radius and host star fractional age remains.

Based on this, we conclude that the radii of close-in Jupitermass giant planets are determined by their present-day equilibrium temperature and semimajor axis, and that the radii of planets increase over time as their equilibrium temperatures increase.

\subsubsection{Selection Effects}

The Effect of Stellar Evolution on the Detectability of Planets: The sample of known TEPs suffers from a broad range of observational selection effects that in principle might explain a preference for finding large planets around evolved stars. As stars evolve, their radii increase, which, for fixed $R_{p}$, $a$, and $M_{\star}$, reduces the transit depth by a factor of $R_{\star}^{-2}$ (reducing their detectability), but increases the duration of the transits by a factor of $R_{\star}$ (increasing their detectability). It also increases the geometrical probability of a planet being seen to transit by a factor of $R_{\star}$ (increasing planet detectability). As stars evolve, they also become more luminous, meaning that at fixed distance they may be monitored with greater photometric precision (further increasing planet detectability). To determine the relative balance of these competing factors for the HAT surveys, for each TEP system discovered by HAT we estimate the relative number of ZAMS stars with the same stellar mass and metallicity around which one could expect to find a planet with the same radius and orbital period and with the same $\mathrm{S} / \mathrm{N}$. To do this, we use the following expression:

$$
N_{\mathrm{ZAMS}} / N_{t}=\frac{V_{\mathrm{ZAMS}}}{V_{t}} \frac{\text { Prob }_{\mathrm{ZAMS}}}{\text { Prob }_{t}}
$$

where $N_{\text {ZAMS }} / N_{t}$ is the relative number of ZAMS planet hosts expected compared to those with age $t, V_{\mathrm{ZAMS}} / V_{t}$ is the relative volume surveyed for ZAMS-equivalent versions of the TEP system (for simplicity we assume a uniform space density of stars), and $\operatorname{Prob}_{\mathrm{ZAMS}} / \mathrm{Prob}_{t}=R_{\star, \mathrm{ZAMS}} / R_{\star, t}$ is the relative transit probability, which is equal to the ratio of the stellar radii. To estimate $V_{\mathrm{ZAMS}} / V_{t}$, we note that for fixed photometric precision, and assuming white-noise-dominated observations, the transit $\mathrm{S} / \mathrm{N}$ of a given TEP scales as the transit depth times the square root of the number of points in transit, or as $\left(R_{\star, \mathrm{ZAMS}} / R_{\star, t}\right)^{-3 / 2}$. If the data are red noise dominated, then the $\mathrm{S} / \mathrm{N}$ scales simply as the transit depth, which would increase $V_{\text {ZAMS }} / V_{t}$. We then determine the $r$ magnitude of stars in the HAT field containing the TEP in question for which the perpoint rms is larger than the rms of the observed TEP light curve by $\left(R_{\star, \mathrm{ZAMS}} / R_{\star, t}\right)^{-3 / 2}$. Accounting for the change in absolute $r$ magnitude between the ZAMS and the present day for the system, this gives us the relative distance of a ZAMSequivalent system for which the transits would be detected with the same $\mathrm{S} / \mathrm{N}$. The cube of this distance is equal to $V_{\text {ZAMS }} / V_{t}$.

In Figure 15 we show $N_{\mathrm{ZAMS}} / N_{t}$ versus $R_{p}$ for HAT planets. For most TEP systems discovered by HAT, including most of the systems with $R_{p}>1.5 R_{\mathrm{J}}$, we have $N_{\mathrm{ZAMS}} / N_{t}>1$. In other 
Table 4

Relative Radial Velocities and Bisector Spans for HAT-P-65 and HAT-P-66

\begin{tabular}{|c|c|c|c|c|c|c|c|}
\hline Star & $\begin{array}{c}\text { BJD } \\
(2,450,000+)\end{array}$ & $\begin{array}{c}\mathrm{RV}^{\mathrm{a}} \\
\left(\mathrm{m} \mathrm{s}^{-1}\right)\end{array}$ & $\begin{array}{c}\sigma_{\mathrm{RV}^{\mathrm{b}}} \\
\left(\mathrm{m} \mathrm{s}^{-1}\right)\end{array}$ & $\begin{array}{c}\mathrm{BS} \\
\left(\mathrm{m} \mathrm{s}^{-1}\right)\end{array}$ & $\begin{array}{c}\sigma_{\mathrm{BS}} \\
\left(\mathrm{m} \mathrm{s}^{-1}\right)\end{array}$ & Phase & Instrument \\
\hline \multicolumn{8}{|c|}{ HAT-P-65 } \\
\hline HAT-P-65 & 5544.74169 & $\cdots$ & $\cdots$ & 19.6 & 29.8 & 0.161 & HIRES \\
\hline HAT-P-65 & 5544.75713 & -27.18 & 7.33 & 59.9 & 26.9 & 0.167 & HIRES \\
\hline HAT-P-65 & 5545.73503 & 65.09 & 6.31 & 20.1 & 10.9 & 0.542 & HIRES \\
\hline HAT-P-65 & 5814.97581 & 54.96 & 5.43 & -23.6 & 7.4 & 0.880 & HIRES \\
\hline HAT-P-65 & 5850.91750 & 39.48 & 5.64 & 43.7 & 11.2 & 0.675 & HIRES \\
\hline HAT-P-65 & 5853.90364 & 67.84 & 5.18 & -0.2 & 6.9 & 0.821 & HIRES \\
\hline HAT-P-65 & 5878.76104 & -74.79 & 5.32 & -1.6 & 4.4 & 0.361 & HIRES \\
\hline HAT-P-65 & 5879.78830 & 79.69 & 5.53 & -1.7 & 5.2 & 0.756 & HIRES \\
\hline HAT-P-65 & 5880.82088 & -70.82 & 5.50 & 0.2 & 6.5 & 0.152 & HIRES \\
\hline HAT-P-65 & 5881.83486 & -15.35 & 6.61 & -21.2 & 8.1 & 0.541 & HIRES \\
\hline HAT-P-65 & 5904.71775 & -81.04 & 4.62 & 2.2 & 7.0 & 0.324 & HIRES \\
\hline HAT-P-65 & 6193.91043 & -34.59 & 5.57 & 5.4 & 5.3 & 0.319 & HIRES \\
\hline HAT-P-65 & 6534.99676 & -65.37 & 5.92 & 5.5 & 12.5 & 0.231 & HIRES \\
\hline \multicolumn{8}{|c|}{ HAT-P-66 } \\
\hline HAT-P-66 & 6991.98631 & -26.96 & 45.68 & $\cdots$ & $\cdots$ & 0.227 & TRES \\
\hline HAT-P-66 & 7061.78904 & 85.42 & 58.94 & $\ldots$ & $\ldots$ & 0.713 & TRES \\
\hline HAT-P-66 & 7064.81417 & 132.13 & 59.20 & $\cdots$ & $\cdots$ & 0.731 & TRES \\
\hline HAT-P-66 & 7079.67198 & 54.56 & 57.38 & $\cdots$ & $\ldots$ & 0.730 & TRES \\
\hline HAT-P-66 & 7110.88787 & -60.24 & 49.59 & $\cdots$ & $\cdots$ & 0.233 & TRES \\
\hline HAT-P-66 & 7112.39413 & 74.38 & 17.40 & 4.6 & 31.3 & 0.740 & Sophie \\
\hline HAT-P-66 & 7136.46148 & 84.58 & 12.70 & 0.8 & 22.9 & 0.838 & Sophie \\
\hline HAT-P-66 & 7146.66832 & -108.08 & 42.72 & $\ldots$ & $\ldots$ & 0.272 & TRES \\
\hline HAT-P-66 & $7166.67684^{c}$ & 170.19 & 73.39 & $\ldots$ & $\ldots$ & 0.004 & TRES \\
\hline HAT-P-66 & 7167.68267 & -22.39 & 54.73 & $\cdots$ & $\cdots$ & 0.343 & TRES \\
\hline HAT-P-66 & 7168.70209 & 116.29 & 42.72 & $\cdots$ & $\cdots$ & 0.686 & TRES \\
\hline HAT-P-66 & 7180.66291 & 190.92 & 75.28 & $\ldots$ & $\cdots$ & 0.710 & TRES \\
\hline HAT-P-66 & 7191.40443 & -132.24 & 20.50 & -42.1 & 36.9 & 0.324 & Sophie \\
\hline HAT-P-66 & $7193.37858^{c}$ & 137.63 & 47.00 & -204.0 & 84.6 & 0.989 & Sophie \\
\hline HAT-P-66 & 7195.40734 & 71.00 & 16.30 & 61.3 & 29.3 & 0.671 & Sophie \\
\hline HAT-P-66 & 7331.65788 & -22.35 & 16.10 & 30.7 & 29.0 & 0.515 & Sophie \\
\hline HAT-P-66 & 7333.68313 & -89.69 & 13.40 & 33.6 & 24.1 & 0.196 & Sophie \\
\hline HAT-P-66 & 7334.67374 & 9.77 & 10.60 & 17.4 & 19.1 & 0.529 & Sophie \\
\hline HAT-P-66 & 7335.68108 & 94.91 & 11.70 & 57.0 & 21.1 & 0.868 & Sophie \\
\hline HAT-P-66 & 7379.14314 & -17.16 & 4.52 & 5.2 & 5.5 & 0.492 & HIRES \\
\hline HAT-P-66 & 7400.62823 & 92.74 & 13.00 & 48.4 & 23.4 & 0.721 & Sophie \\
\hline HAT-P-66 & 7402.71917 & -53.77 & 17.00 & -29.5 & 30.6 & 0.424 & Sophie \\
\hline HAT-P-66 & 7403.52312 & 91.28 & 18.30 & -6.8 & 32.9 & 0.695 & Sophie \\
\hline HAT-P-66 & 7404.61059 & -18.72 & 17.00 & -3.1 & 30.6 & 0.061 & Sophie \\
\hline HAT-P-66 & 7405.56638 & -60.84 & 14.00 & 25.1 & 25.2 & 0.382 & Sophie \\
\hline HAT-P-66 & 7412.16895 & 51.53 & 3.81 & 4.8 & 8.7 & 0.604 & HIRES \\
\hline HAT-P-66 & 7413.08806 & 61.91 & 3.34 & -3.3 & 4.6 & 0.913 & HIRES \\
\hline HAT-P-66 & 7413.94835 & -67.66 & 2.93 & 5.4 & 3.0 & 0.202 & HIRES \\
\hline HAT-P-66 & 7415.04674 & 37.83 & 3.20 & -14.4 & 5.7 & 0.572 & HIRES \\
\hline HAT-P-66 & 7422.94521 & $\cdots$ & $\cdots$ & 2.3 & 2.2 & 0.230 & HIRES \\
\hline
\end{tabular}

Notes.

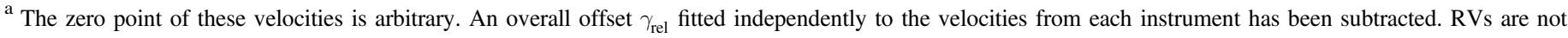
measured for the $\mathrm{I}_{2}$-free HIRES template spectra, but spectral line BSs are measured for these spectra.

${ }^{b}$ Internal errors excluding the component of astrophysical jitter considered in Section 3.3.

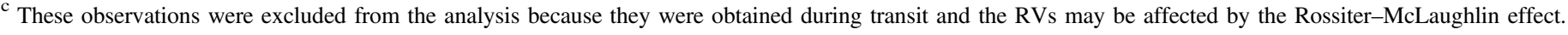

words, the greater transit depths expected for ZAMS systems more than compensate for the lower luminosities, shorterduration transits, and lower transit probabilities. Thus, from a pure transit-detection point of view, we should expect to be more sensitive to TEPs around ZAMS stars than to TEPs around stars with the measured host star ages.

Put another way, while selection effects may lead to fewer small planets being found around older stars (missing planets in the upper left corner of Figure 12), based on the estimate in Figure 15, selection effects due to transit detectability do not explain why we find fewer large planets around unevolved stars (missing planets in the lower right corner of Figure 12). If the occurrence rate of large-radius planets is independent of host star age, or if it is larger for unevolved stars than for evolved stars, we would expect to have found more large planets around unevolved stars than evolved stars. 
Table 5

Stellar Parameters for HAT-P-65 and HAT-P-66 ${ }^{\mathrm{a}}$

\begin{tabular}{|c|c|c|c|}
\hline Parameter & $\begin{array}{l}\text { HAT-P-65 } \\
\text { Value }\end{array}$ & $\begin{array}{c}\text { HAT-P-66 } \\
\text { Value }\end{array}$ & Source \\
\hline \multicolumn{4}{|c|}{ Astrometric properties and cross-identifications } \\
\hline 2MASS-ID & $21033731+1159218$ & $10021743+5357031$ & $\ldots$ \\
\hline GSC-ID & GSC 1111-00383 & GSC 3814-00307 & $\ldots$ \\
\hline R.A. (J2000) & $21^{\mathrm{h}} 03^{\mathrm{m}} 37^{\mathrm{s}} .44$ & $10^{\mathrm{h}} 02^{\mathrm{m}} 17^{\mathrm{s}} .52$ & 2MASS \\
\hline Decl. (J2000) & $+11^{\circ} 59^{\prime} 21^{\prime \prime} 9$ & $+53^{\circ} 57^{\prime} 03^{\prime \prime} 1$ & 2MASS \\
\hline$\mu_{\text {R.A. }}\left({\left.\text { mas } \mathrm{yr}^{-1}\right)}^{-1}\right.$ & $5.5 \pm 1.9$ & $-9.2 \pm 1.8$ & UCAC4 \\
\hline$\mu_{\text {Decl. }}\left(\operatorname{mas~yr}^{-1}\right)$ & $-4.0 \pm 1.9$ & $-11.4 \pm 2.4$ & UCAC4 \\
\hline \multicolumn{4}{|c|}{ Spectroscopic properties } \\
\hline$T_{\text {eff } \star}(\mathrm{K})$ & $5835 \pm 51$ & $6002 \pm 50$ & $\mathrm{SPC}^{\mathrm{b}}$ \\
\hline $\log g_{\star}(\mathrm{cgs})$ & $4.18 \pm 0.10$ & $3.96 \pm 0.10$ & $\mathrm{SPC}^{\mathrm{c}}$ \\
\hline$[\mathrm{Fe} / \mathrm{H}]$ & $0.100 \pm 0.080$ & $0.035 \pm 0.080$ & SPC \\
\hline$v \sin i\left(\mathrm{~km} \mathrm{~s}^{-1}\right)$ & $7.10 \pm 0.50$ & $7.57 \pm 0.50$ & SPC \\
\hline$v_{\mathrm{mac}}\left(\mathrm{km} \mathrm{s}^{-1}\right)$ & 1.0 & 1.0 & Assumed \\
\hline$v_{\text {mic }}\left(\mathrm{km} \mathrm{s}^{-1}\right)$ & 2.0 & 2.0 & Assumed \\
\hline$\gamma_{\mathrm{RV}}\left(\mathrm{m} \mathrm{s}^{-1}\right)$ & $-47.77 \pm 0.10$ & $7.97 \pm 0.10$ & TRES $^{\mathrm{d}}$ \\
\hline$S_{\mathrm{HK}}$ & $\cdots$ & $\ldots$ & HIRES \\
\hline $\log R_{\mathrm{HK}}^{\prime}$ & $\cdots$ & $\cdots$ & HIRES \\
\hline \multicolumn{4}{|l|}{ Photometric properties } \\
\hline$B$ (mag) & $13.818 \pm 0.021$ & $13.552 \pm 0.027$ & APASS $^{\mathrm{e}}$ \\
\hline$V$ (mag) & $13.145 \pm 0.029$ & $12.993 \pm 0.052$ & $\mathrm{APASS}^{\mathrm{e}}$ \\
\hline$I$ (mag) & $12.46 \pm 0.10$ & $12.339 \pm 0.084$ & TASS Mark IV ${ }^{\mathrm{f}}$ \\
\hline$g$ (mag) & $13.445 \pm 0.016$ & $13.209 \pm 0.021$ & APASS \\
\hline$r(\mathrm{mag})$ & $12.948 \pm 0.033$ & $12.859 \pm 0.064$ & APASS $^{\mathrm{e}}$ \\
\hline$i$ (mag) & $12.784 \pm 0.097$ & $12.771 \pm 0.064$ & APASS $^{\mathrm{e}}$ \\
\hline$J$ (mag) & $11.892 \pm 0.026$ & $12.001 \pm 0.022$ & 2MASS \\
\hline$H$ (mag) & $11.604 \pm 0.022$ & $11.735 \pm 0.022$ & 2MASS \\
\hline$K_{s}(\mathrm{mag})$ & $11.528 \pm 0.025$ & $11.675 \pm 0.022$ & 2MASS \\
\hline \multicolumn{4}{|l|}{ Derived properties } \\
\hline$M_{\star}\left(M_{\odot}\right)$ & $1.212 \pm 0.050$ & $1.255_{-0.054}^{+0.107}$ & $\mathrm{YY}+\rho_{\star}+\mathrm{SPC}^{\mathrm{g}}$ \\
\hline$R_{\star}\left(R_{\odot}\right)$ & $1.860 \pm 0.096$ & $1.881_{-0.095}^{+0.151}$ & $\mathrm{YY}+\rho_{\star}+\mathrm{SPC}$ \\
\hline $\log g_{\star}(\mathrm{cgs})$ & $3.983 \pm 0.035$ & $3.993 \pm 0.045$ & $\mathrm{YY}+\rho_{\star}+\mathrm{SPC}$ \\
\hline$\rho_{\star}\left(\mathrm{g} \mathrm{cm}^{-3}\right)$ & $0.266 \pm 0.035$ & $0.269 \pm 0.040$ & Light curves \\
\hline$L_{\star}\left(L_{\odot}\right)$ & $3.59 \pm 0.40$ & $4.12_{-0.46}^{+0.71}$ & $\mathrm{YY}+\rho_{\star}+\mathrm{SPC}$ \\
\hline$M_{V}(\mathrm{mag})$ & $3.43 \pm 0.13$ & $3.26 \pm 0.15$ & $\mathrm{YY}+\rho_{\star}+\mathrm{SPC}$ \\
\hline$M_{K}(\mathrm{mag}, \mathrm{ESO})$ & $1.93 \pm 0.12$ & $1.86 \pm 0.14$ & $\mathrm{YY}+\rho_{\star}+\mathrm{SPC}$ \\
\hline Age (Gyr) & $5.46 \pm 0.61$ & $4.66_{-1.12}^{+0.52}$ & $\mathrm{YY}+\rho_{\star}+\mathrm{SPC}$ \\
\hline$A_{V}(\mathrm{mag})$ & $0.090 \pm 0.052$ & $0.0000 \pm 0.0062$ & $\mathrm{YY}+\rho_{\star}+\mathrm{SPC}$ \\
\hline Distance (pc) & $841 \pm 45$ & $927_{-49}^{+75}$ & $\mathrm{YY}+\rho_{\star}+\mathrm{SPC}$ \\
\hline
\end{tabular}

Notes. For both systems the fixed-circular-orbit model has a higher Bayesian evidence than the eccentric-orbit model. We therefore assume a fixed circular orbit in generating the parameters listed here.

${ }^{a}$ We adopt the IAU 2015 Resolution B3 nominal values for the solar and Jovian parameters (Prša et al. 2016) for all of our calculations, taking $R_{\mathrm{J}}$ to be the nominal equatorial radius of Jupiter. Where necessary we assume $G=6.6408 \times 10^{-11} \mathrm{~m}^{3} \mathrm{~kg}^{-1} \mathrm{~s}^{-1}$. Because Yi et al. (2001) do not specify the assumed value for $G$ or $M_{\odot}$, we take the stellar masses from these isochrones at face value without conversion. Any discrepancy results in an error that is less than $1 \%$, which is well below the observational uncertainty. We note that the standard values assumed in prior HAT planet discovery papers are very close to the nominal values adopted here. In all cases the conversion results in changes to measured parameters that are indetectable at the level of precision to which they are listed.

${ }^{b}$ SPC-Stellar Parameter Classification procedure for the analysis of high-resolution spectra (Buchhave et al. 2012), applied to the TRES spectra of HAT-P-65 and the Keck/HIRES spectra of HAT-P-66. These parameters rely primarily on SPC but also have a small dependence on the iterative analysis incorporating the isochrone search and global modeling of the data.

${ }^{\mathrm{c}}$ The spectroscopically determined value of $\log g_{\star}$ is from our initial SPC analysis, where $T_{\text {eff }}, \log g_{\star},[\mathrm{Fe} / \mathrm{H}]$, and $v$ sin $i$ were all varied. Systematic errors are common when all four parameters are varied. The adopted values for $T_{\mathrm{eff} \star},[\mathrm{Fe} / \mathrm{H}]$, and $v \sin i$ stem from a second iteration of SPC, where $\log g_{\star}$ is fixed to the value determined through the light-curve modeling and isochrone comparison. This value is listed under the "Derived properties" section of the table.

${ }^{\mathrm{d}}$ In addition to the uncertainty listed here, there is a $\sim 0.1 \mathrm{~km} \mathrm{~s}^{-1}$ systematic uncertainty in transforming the velocities to the IAU standard system.

e From APASS DR6 as listed in the UCAC4 catalog (Zacharias et al. 2013).

${ }^{\mathrm{f}}$ Droege et al. (2006).

${ }^{\mathrm{g}} \mathrm{YY}+\rho_{\star}+\mathrm{SPC}=$ based on the YY isochrones (Yi et al. 2001), $\rho_{\star}$ as a luminosity indicator, and the SPC results.

The Effect of Stellar Evolution on the Ability to Confirm Planets: Other observational selection effects may still be at play. If the orbits of these planets shrink over time due to tidal evolution, then the transit probability and the fraction of points in transit both increase in time by more that what we estimated. Beyond simply detecting the transits, further selections are 
HAT

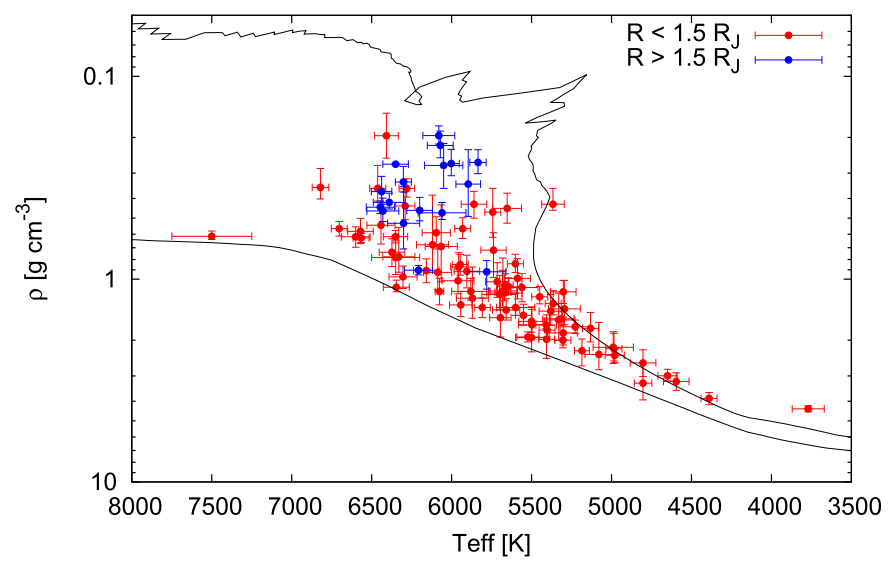

Kepler

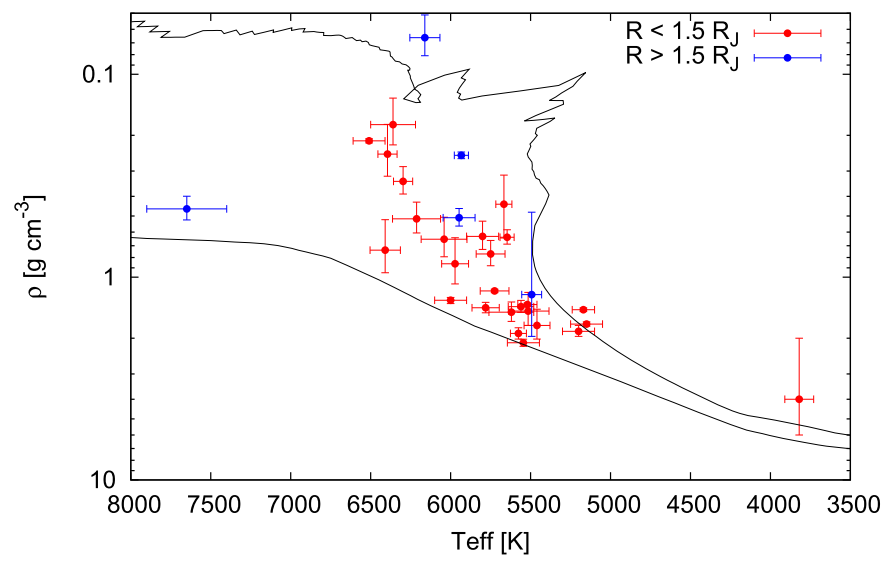

WASP

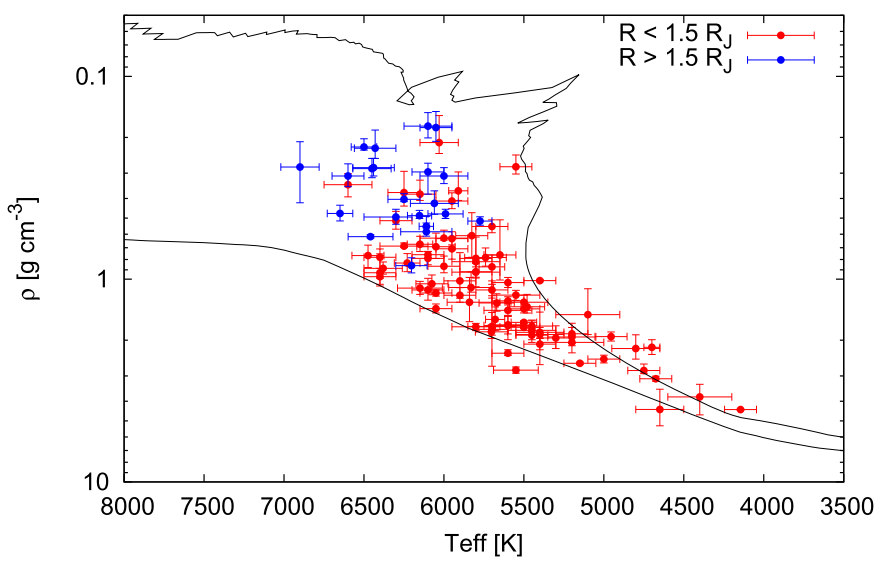

TrES + KELT

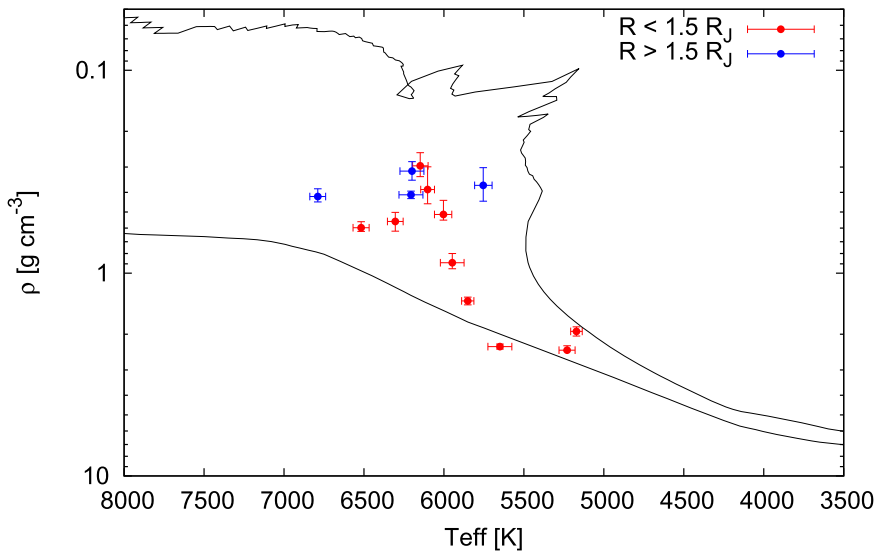

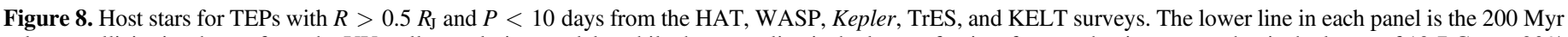

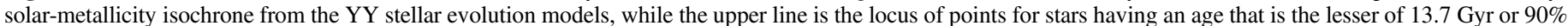

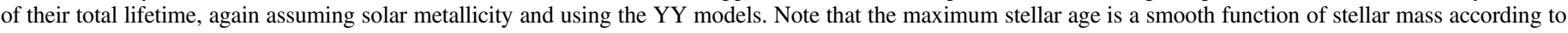

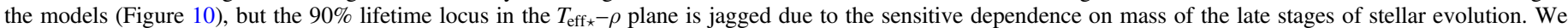

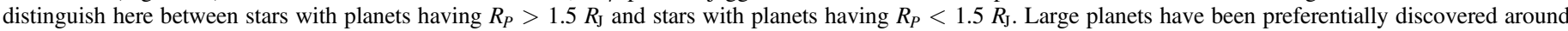

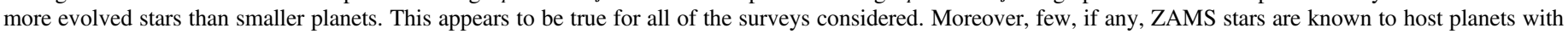
$R_{P}>1.5 R_{\mathrm{J}}$.

imposed in the follow-up program carried out to confirm the planets. Figure 16 compares the present-day effective temperature and $v \sin i$ for HAT TEPs to the expected values on the ZAMS (estimated as discussed below). For all of the $R_{p}>1.5$ $R_{\mathrm{J}}$ planets found by HAT the host star had a higher $T_{\text {eff } \star}$ on the ZAMS than at the present day. The most extreme case is HATP-7, which had an estimated ZAMS effective temperature of $6860 \mathrm{~K}$ compared to its present-day temperature of $6350 \pm 100 \mathrm{~K}$. While precision RVs are more challenging for early $F$ dwarfs than for later $F$ dwarfs, the ZAMS temperatures of the hosts of the largest TEPs found by HAT are still within the range where we carry out follow-up observations (we do not follow up hosts of spectral type A or earlier if they are faint stars with $V \gtrsim 13$ ).

An additional potential selection effect relates to the stellar rotation. Neglecting tidal interactions between the stars and planets, the host stars would have had higher projected rotation velocities at ZAMS, primarily resulting from their lower moments of inertia (most of the hosts of the largest planets found by HAT have radiative envelopes, or would have had them for much of their main-sequence lifetimes, and thus would not lose substantial angular momentum from magnetized stellar winds). Roughly speaking, we expect $v \sin i \propto R_{\star}^{-1}$ (assuming $I \propto R_{\star}^{2}$ ). The most rapidly rotating ZAMS host is HAT-P-41, for which we estimate a ZAMS rotation velocity of $24 \mathrm{~km} \mathrm{~s}^{-1}$, which is still well within the range where we continue follow-up (we do not follow up hosts with $v \sin i \gtrsim 50 \mathrm{~km} \mathrm{~s}^{-1}$ if they are around faint stars with $V \gtrsim 13$ ). Thus, while precise RVs would be somewhat more challenging for ZAMS planet hosts than for moderately evolved hosts, these factors are unlikely to be responsible for the lack of highly inflated planets discovered to date around stars close to ZAMS.

Correcting the Correlation Coefficient for Observational Selections: In order to determine quantitatively how selection effects impact the correlation measured between $R_{p}$ and $\tau$, we follow Efron \& Petrosian (1999) in calculating a modified Kendall correlation coefficient that is applicable to data suffering a nontrivial truncation. The procedure is as follows. We will call the observed data points $\left(R_{P, i}, \tau_{i}\right)$ and $\left(R_{P, j}, \tau_{j}\right)$, with $i \neq j$, comparable if each point falls within the other point's selection range. Here point $j$ is within the selection range for point $i$ if, holding everything else constant, we could still have discovered the planet around star $i$ if the system had values of $\left(R_{P, i}, \tau_{j}\right),\left(R_{P, j}, \tau_{i}\right)$, or $\left(R_{P, j}, \tau_{j}\right)$ instead of $\left(R_{P, i}, \tau_{i}\right)$. Letting $\mathcal{J}$ be the set of all comparable pairs and $N_{p}$ be the total 
HAT

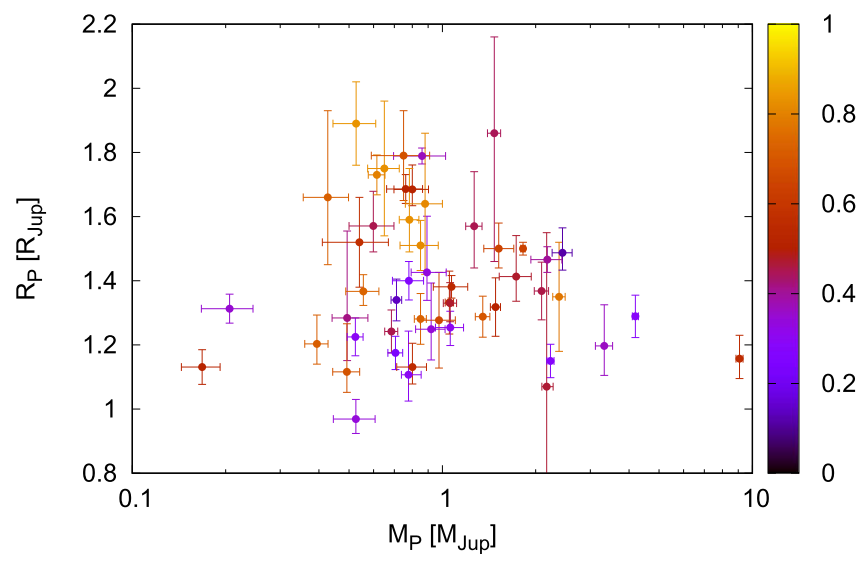

Kepler

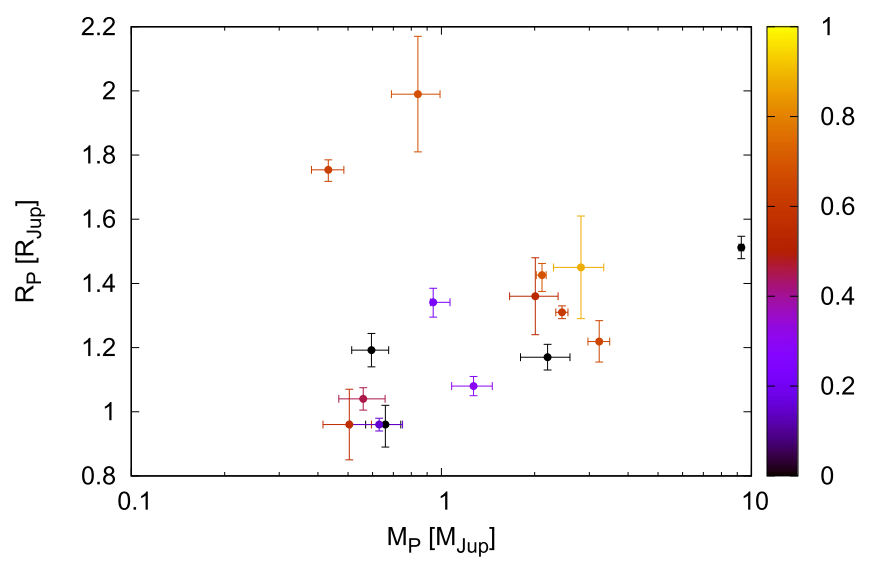

WASP

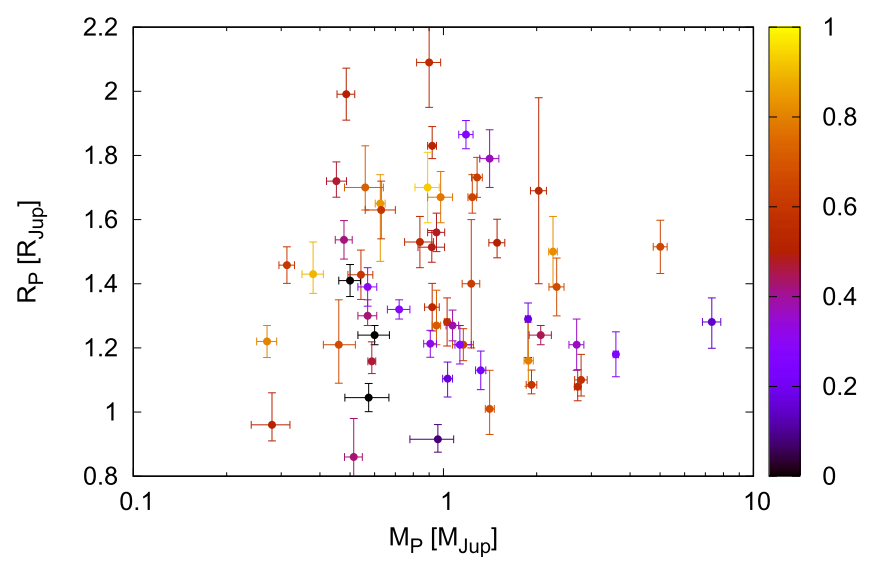

TrES + KELT

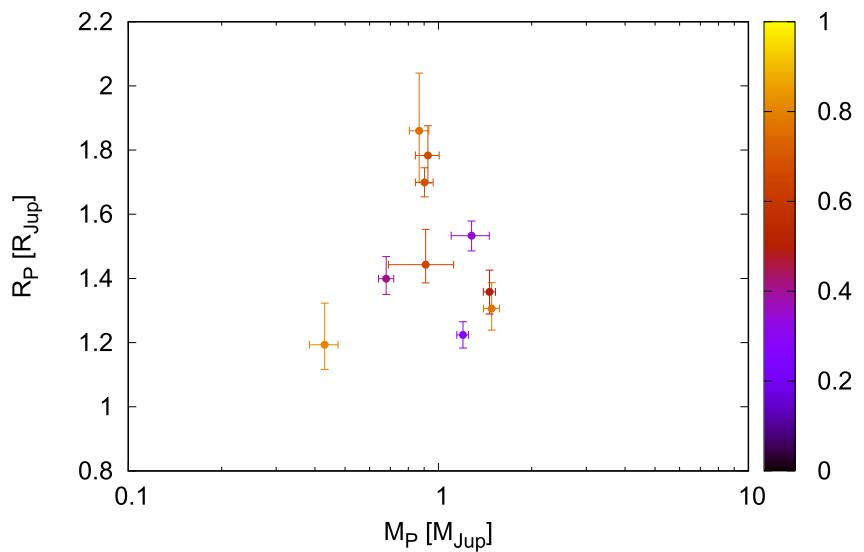

Figure 9. Mass-radius relation for TEPs from HAT (top left), WASP (top right), Kepler (bottom left), and TrES and KELT (bottom right) with $R>0.5 R_{\mathrm{J}}$ and $P<10$ days around stars with total lifetimes $t_{\text {tot }}<10 \mathrm{Gyr}$. The color scale for each point indicates the fractional age of the system (taken to be $\tau=(t-200 \mathrm{Myr}) /\left(t_{\mathrm{tot}}-200 \mathrm{Myr}\right)$, where $t$ is the age determined from the YY isochrones using $T_{\text {eff }}, \rho_{\star}$, and $[\mathrm{Fe} / \mathrm{H}]$ and $t_{\text {tot }}$ is the maximum age in the YY models for a star with the same mass and $[\mathrm{Fe} / \mathrm{H}])$. A handful of stars with bulk densities indicating very young ages show up as black points in the figure. The largest planets are found almost exclusively around moderately evolved $(\tau \gtrsim 0.5)$ stars.

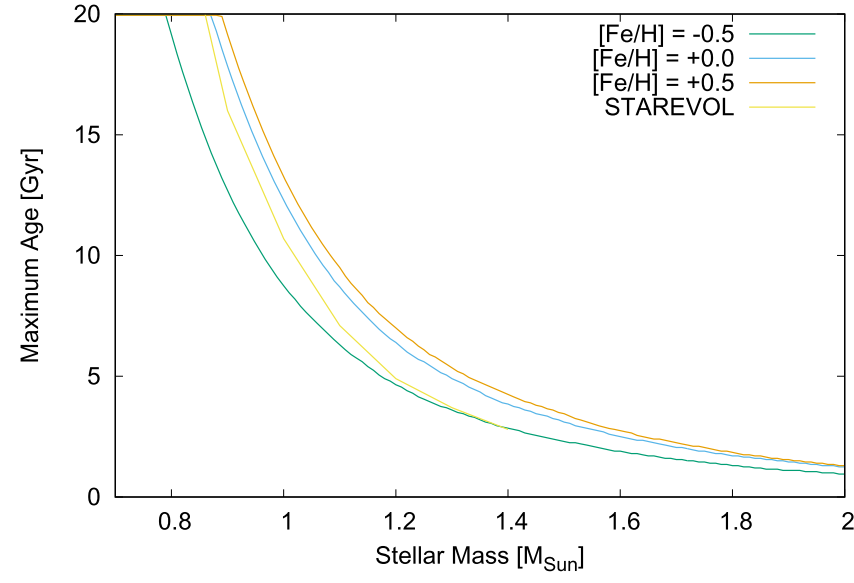

Figure 10. Maximum age of a star as a function of its mass based on interpolating within the YY isochrones. These are shown for three representative metallicities. The maximum age is artificially capped at 19.95 Gyr, which is the largest age at which the models are tabulated. For stars with $M \gtrsim 0.85 M_{\odot}$, which have maximum ages below this artificial cap, there is a smooth power-law dependence between the maximum age and mass. We use this relation to estimate the fractional age $\tau$ of a planetary system. For comparison we also show the terminal main-sequence age as a function of stellar mass from the STAREVOL evolution tracks (Charbonnel \& Palacios 2004; Lagarde et al. 2012), taken from Table B.6 of Santerne et al. (2016). number of such pairs, the modified Kendall correlation coefficient is then given by

$$
r_{K}=\frac{1}{N_{p}} \sum_{(i, j) \in \mathcal{J}} \operatorname{sign}\left(\left(R_{P, i}-R_{P, j}\right)\left(\tau_{i}-\tau_{j}\right)\right) .
$$

For uncorrelated data $r_{K}$ has an expected value of 0 , whereas perfectly correlated data have $r_{K}=1$ and perfectly anticorrelated data have $r_{K}=-1$. To determine the probability of finding $\left|r_{K}\right|>\left|r_{K \text {,observed }}\right|$, it is necessary to carry out bootstrap simulations. To do this, we calculate $r_{K}$ for $N_{\text {sim }}$ simulated data sets, and for each simulated data set we randomly select $N$ values of $i$, with replacement, from the observed samples, adopt $R_{P, i}$ for each simulated point, and associate with it a value of $\tau$ drawn at random from the set of points that are comparable to $i$ (including $i$ itself in this case).

The primary challenge in calculating Equation (5) for the observed sample of close-in giant planets is to determine the set of comparable pairs. We do this for the HAT planets by subtracting the observed transit signal from the survey light curve, rescaling the scatter to match the expected change in rms due to the change in the stellar luminosity with age, adding the expected transit signal given the new trial planetary and stellar radii, but assuming the original ephemeris and orbital 


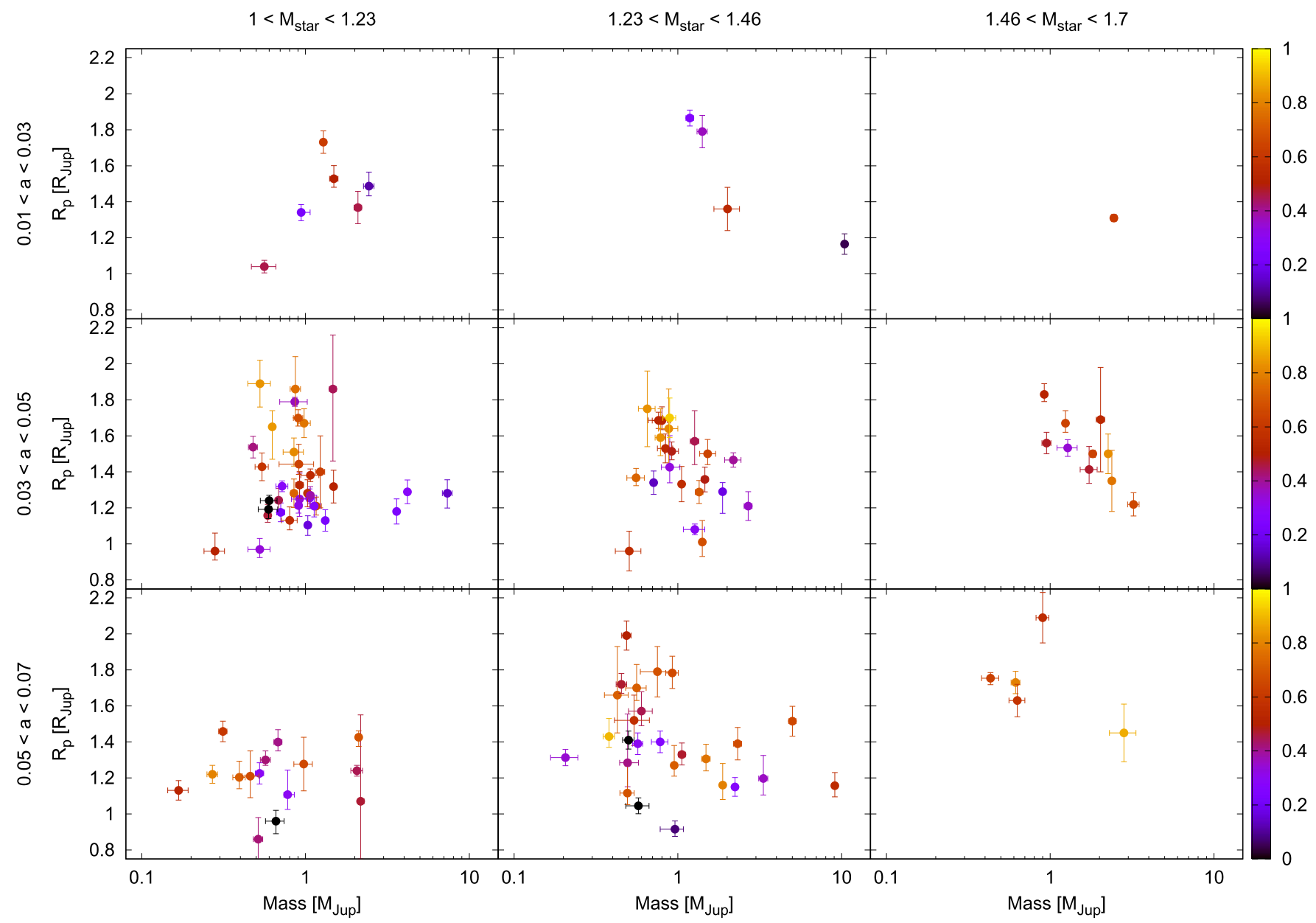

Figure 11. Similar to Figure 9, but here we combine all of the data from the different surveys and show the mass-radius relation for different host star mass ranges (the selections are shown at the top of each column in solar mass units) and orbital semimajor axes (the selections are shown to the left of each row in au). The overall range of semimajor axis and stellar mass shown here is chosen to encompass the sample of well-characterized highly inflated planets with $R>1.5 R_{\mathrm{J}}$ around stars with total lifetimes $t_{\mathrm{tot}}<10 \mathrm{Gyr}$. Within a given panel the largest planets tend to be found around more evolved stars. This is the opposite of what one would expect if high irradiation slows a planet's contraction but does not supply energy deep enough into the interior of the planet to reinflate as the luminosity of its host star increases.

inclination, and using BLS to determine whether or not the transit could be recovered. Using the set of comparable pairs determined in this fashion, we find $r_{K}=0.228$, with a $2.88 \%$ false-alarm probability, based on the bootstrap simulations (or $r_{K}=0.284$ with a $2.92 \%$ false-alarm probability when restricted to planets with $0.4 M_{\mathrm{J}}<M_{p}<2.0 M_{\mathrm{J}}$ ). For comparison, if we ignore the selection effects and assume that all points are comparable, we find $r_{K}=0.235$ with a false-alarm probability of $1.52 \%$ (or $r_{K}=0.297$ with a $0.87 \%$ false-alarm probability when restricted to planets with $0.4 M_{\mathrm{J}}<M_{p}<2.0 M_{\mathrm{J}}$ ). The false-alarm probabilities in the latter case are essentially the same as what was reported above using the Spearman rank-order correlation test instead of the Kendall test, demonstrating the consistency of the two methods.

We conclude that while observational selections do slightly bias the measured correlation between $R_{p}$ and $\tau$ for HAT, the effect is small. While we cannot determine the set of comparable pairs for WASP, the selections are likely very similar to HAT, and we expect the effect on the measured correlation of accounting for observational selections to be similarly small. We therefore expect that the full combined set of planets would still exhibit a highly significant correlation between $R_{p}$ and $\tau$, even after accounting for observational selections.

\subsubsection{Systematic Errors in Stellar Parameters}

The radii of TEPs are not measured directly, but rather are measured relative to the stellar radii, which in turn are determined by matching the effective temperatures, stellar densities, and stellar metallicities to models (either theoretical stellar evolution models, as done, for example, for most HAT systems, or by utilizing empirical models calibrated with stellar eclipsing binary systems, as has been done for many WASP systems). Any systematic error in the stellar radius would lead to a proportional error in the planet radius, and the fact that the largest planets are more commonly found around the most evolved (and largest) stars is what one would expect to see if there were significant unaccounted-for systematic errors. Here we consider a variety of potential systematic errors and argue that none of these are responsible for the observed correlation.

Eccentricity: One potentially important source of systematic errors in this respect is the planetary eccentricity, which is constrained primarily by the RV data, and which is needed to determine the stellar density from the measured transit duration, impact parameter, and radius ratio. The host stars of the largest-radius planets are among the hottest, fastest-rotating, and highest-jitter stars around which transiting planets have been found (e.g., Figure 16, and Hartman et al. 2011c). For 
HAT

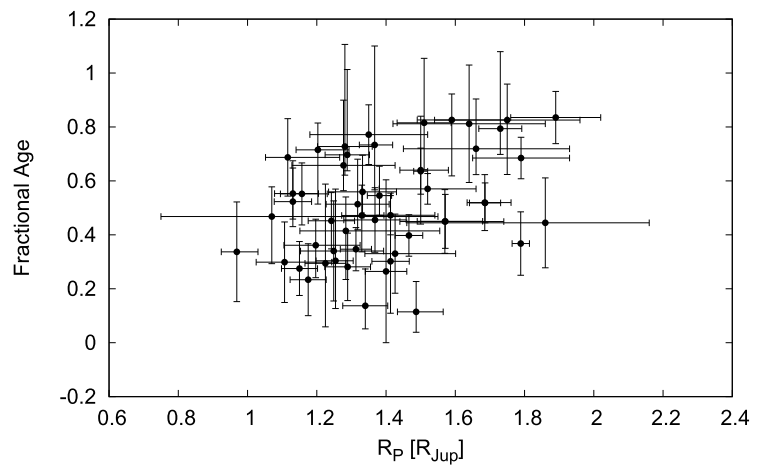

Kepler

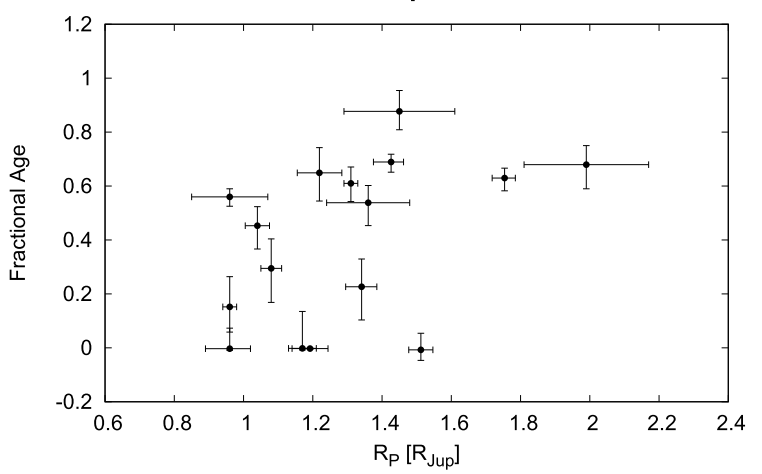

WASP

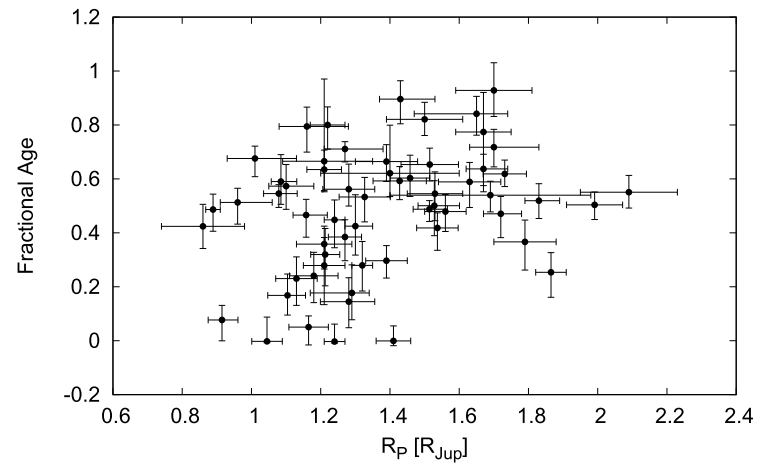

TrES+KELT

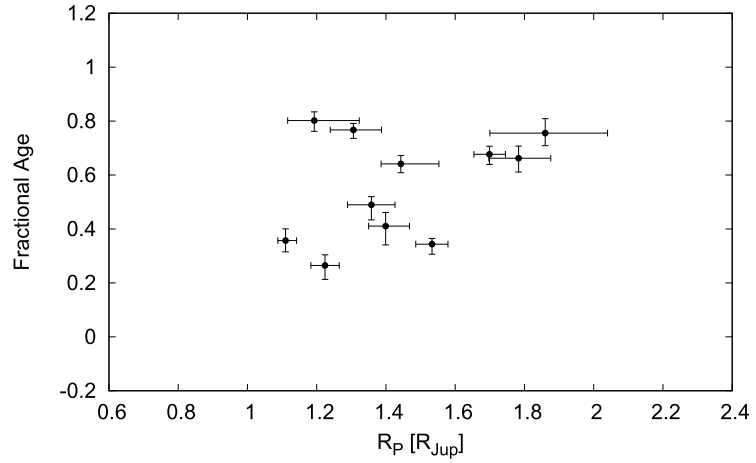

Combined

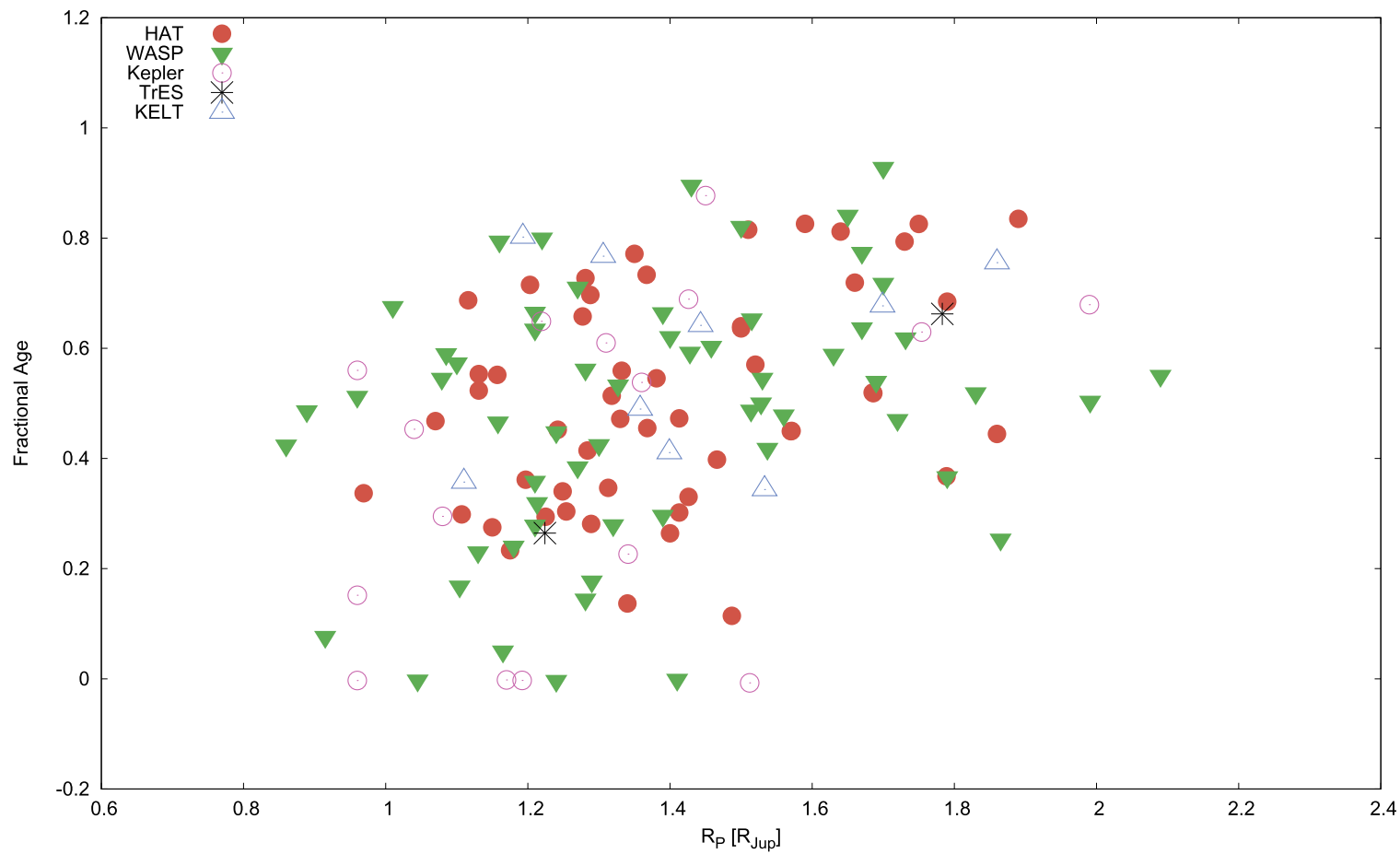

Figure 12. Top: fractional isochrone-based age of the system (see Figure 9) vs. the planetary radius, shown separately for TEP systems discovered by HAT (left) and WASP (right). We only show systems with $P<10$ days and $t_{\text {tot }}<10 \mathrm{Gyr}$. Both the HAT and WASP samples have positive correlations between $R_{P}$ and $\tau$. For HAT a Spearman nonparametric rank-order correlation test gives a correlation coefficient of 0.344 with a $1.4 \%$ false-alarm probability. For the WASP sample we find a correlation coefficient of 0.277 and a false-alarm probability of 3.5\%. Middle: same as the top, but here we show planets from Kepler, TrES, and KELT, with the same selections applied. Bottom: same as the top, but here we combine data from all of the surveys. The combined data set has a correlation coefficient of 0.347 and a falsealarm probability $0.0041 \%$. 
HAT

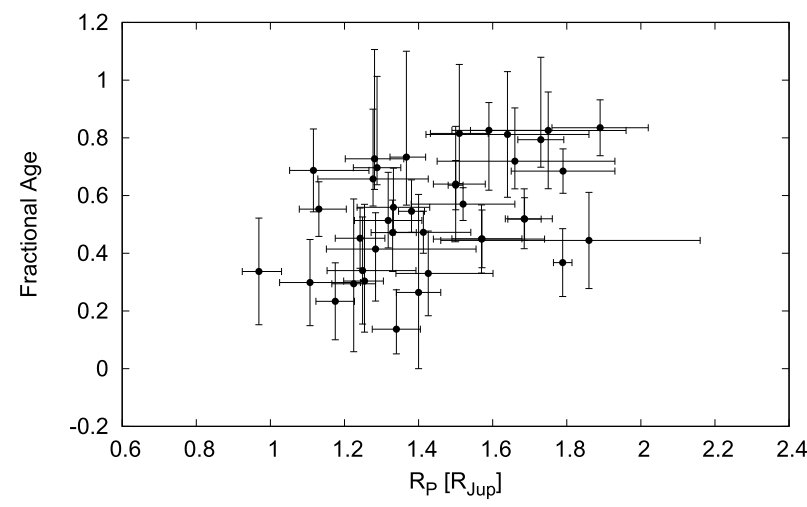

Kepler

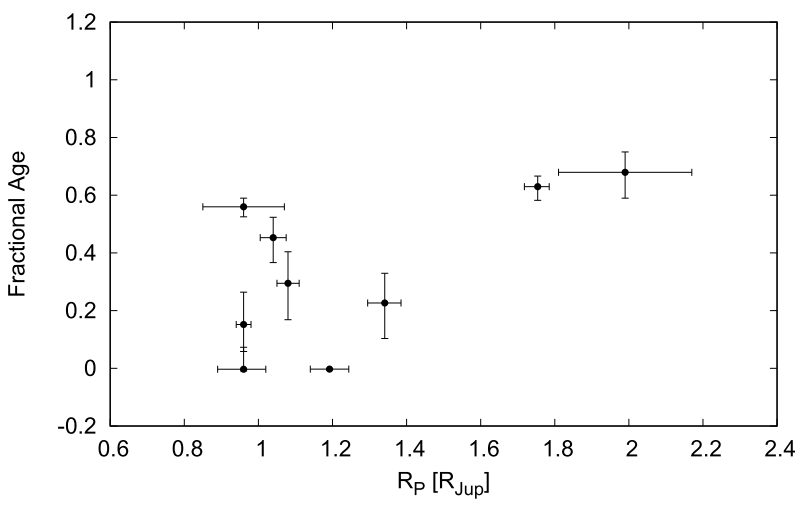

WASP

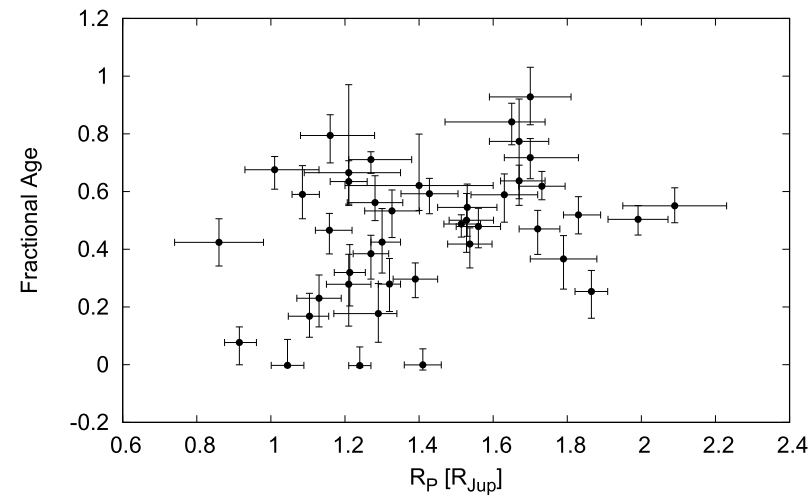

TrES+KELT

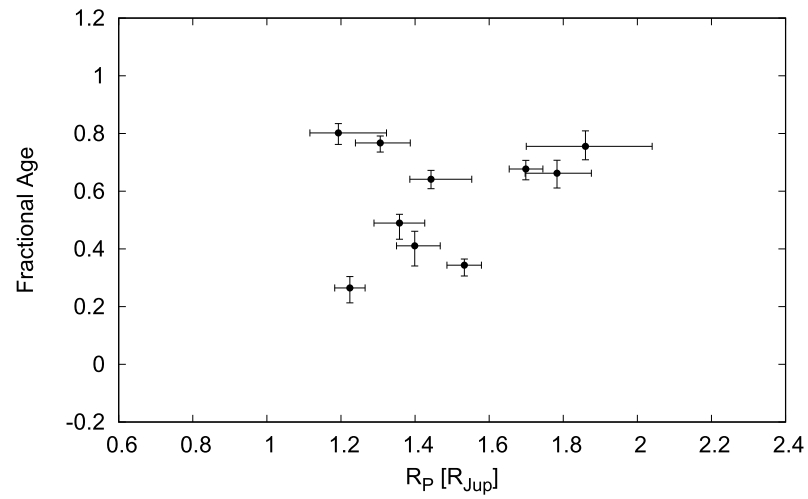

Combined

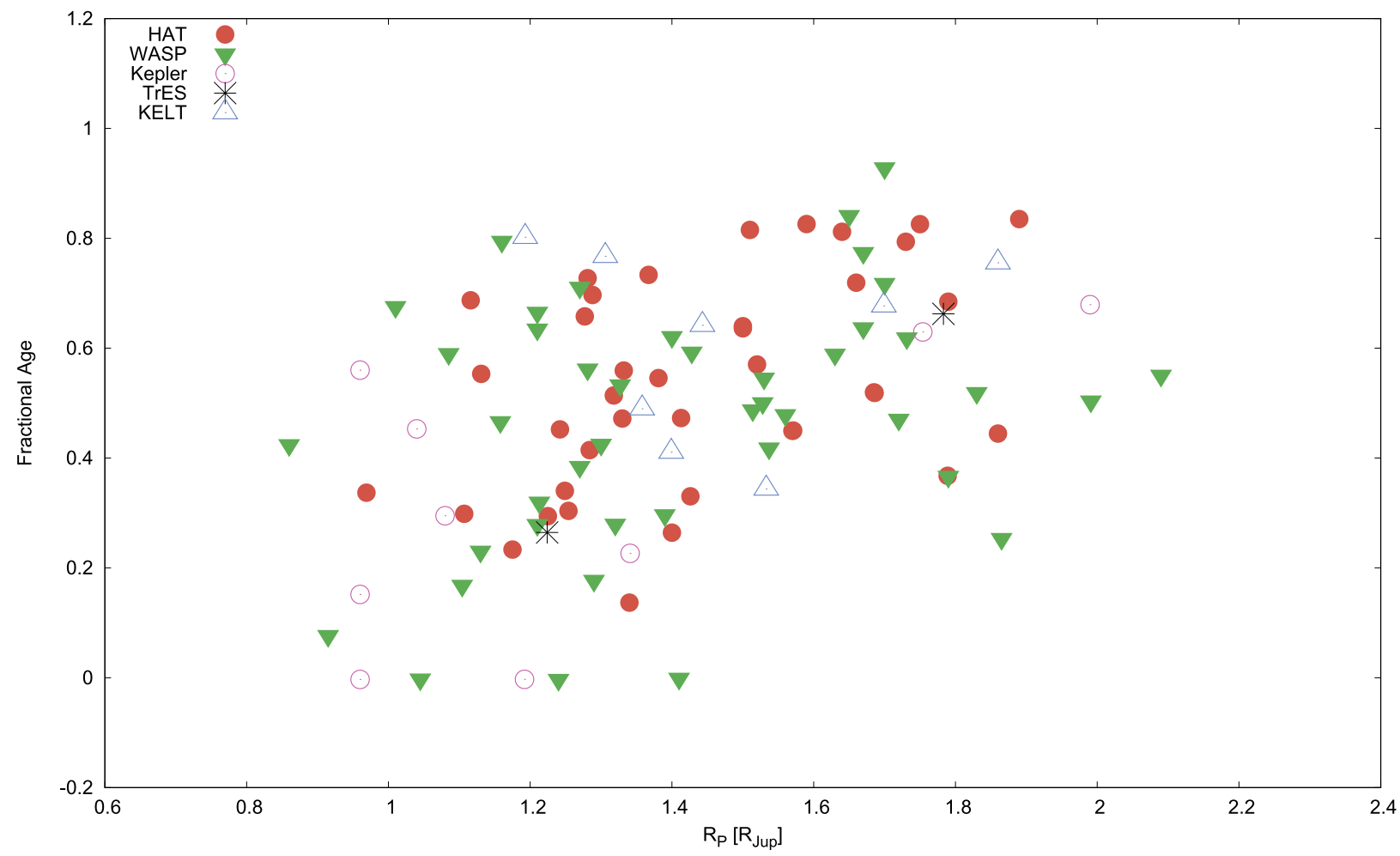

Figure 13. Same as Figure 12, but here we only consider systems with planets having masses in the range $0.4 M_{\mathrm{J}}<M_{p}<2.0 M_{\mathrm{J}}$, which is roughly the radius range over which highly inflated planets have been discovered. In this case the Spearman nonparametric rank-order correlation test gives a correlation coefficient of 0.428 with a $0.84 \%$ false-alarm probability. For the WASP sample we find a correlation coefficient of 0.273 and a false-alarm probability of $7.7 \%$. The combined sample has a correlation coefficient of 0.398 and a false-alarm probability of $0.0068 \%$. 


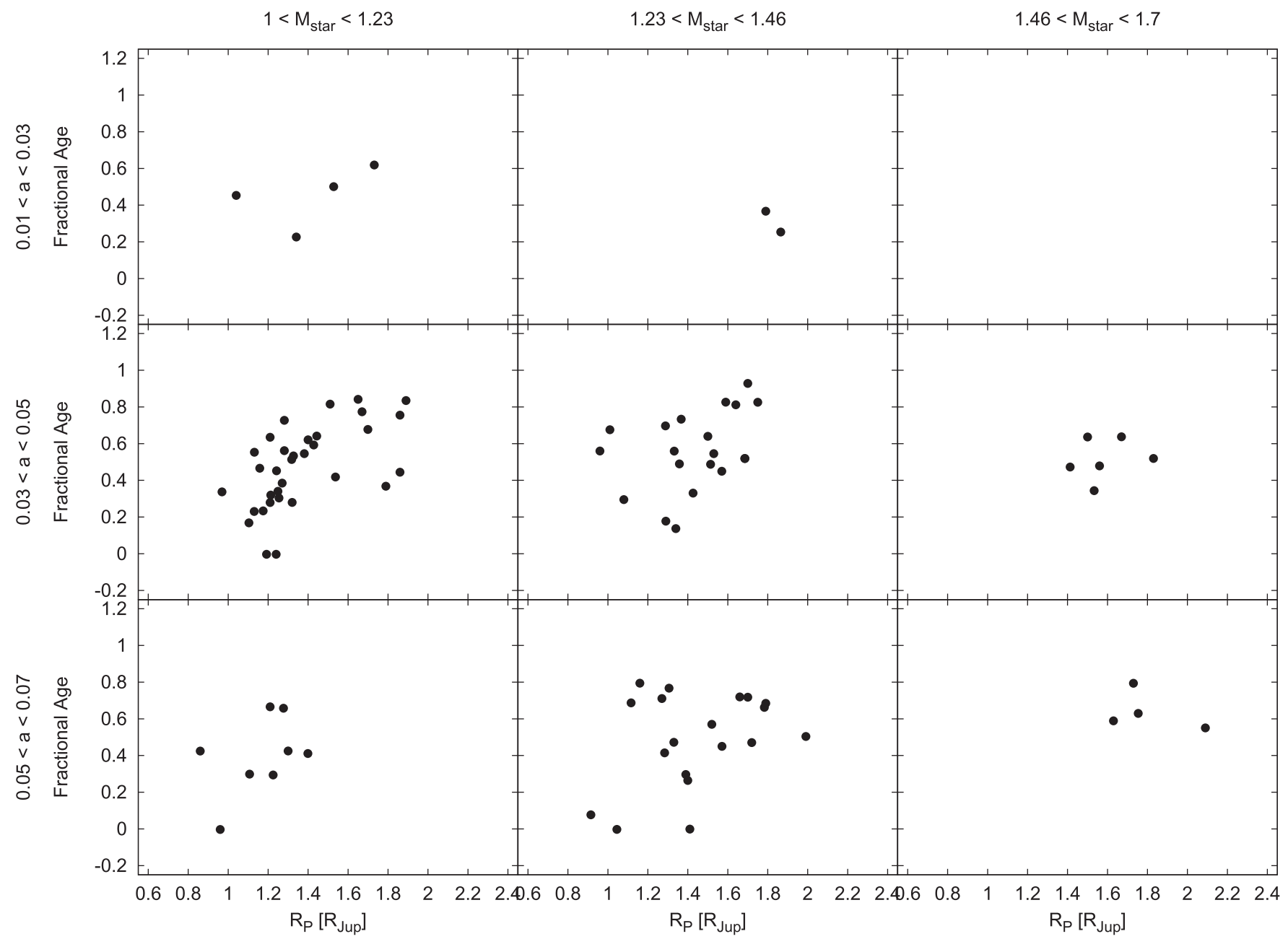

Figure 14. Similar to Figures 11 and 13, but here we combine all of the data from the different surveys and show the fractional isochrone-based age vs. planet radius for different host star mass ranges (the selections are shown at the top of each column in solar mass units) and orbital semimajor axes (the selections are shown to the left of each row in au). The overall range of semimajor axis and stellar mass shown here is chosen to encompass the sample of well-characterized highly inflated planets with $R>1.5 R_{\mathrm{J}}$ around stars with total lifetimes $t_{\text {tot }}<10 \mathrm{Gyr}$. We also restrict the sample to planets with $0.4 M_{\mathrm{J}}<M_{P}<2.0 M_{\mathrm{J}}$.

these systems the eccentricity is typically poorly constrained, and circular orbits have often been adopted. ${ }^{19}$ If the systems were actually highly eccentric, with transits near apastron, then the stellar densities would be higher than what was inferred assuming circular orbits, and the stellar and planetary radii would be smaller than what has been estimated. There are, however, several large planets transiting moderately evolved stars for which secondary eclipses have been observed, providing tight constraints on the eccentricity (e.g., TrES-4b, Knutson et al. 2009; WASP-12b, Campo et al. 2011; HAT-P32b, Zhao et al. 2014; WASP-48b, O'Rourke et al. 2014). Moreover, the most inflated planets are on short-period orbits, where we expect circularization. This expectation has been observationally verified in cases where sufficiently high precision RVs have been possible, or when secondary eclipse follow-up observations have been made. We also note that at least for the majority of the very large radius HAT planets, when the eccentricity is allowed to vary, the planet and stellar radii determined from the median of the posterior distributions

\footnotetext{
19 If circular orbits are not adopted, then there is a bias toward overestimating the eccentricity, as shown by Lucy \& Sweeney (1971). This bias may affect some of the earliest-discovered planets especially.
}

are found to be larger than when the eccentricity is fixed to zero.

Impact Parameter: Another potential source of systematic error is if the impact parameter is in error, perhaps due to an incorrect treatment of limb darkening (e.g., Espinoza \& Jordán 2015). Errors in the impact parameter will translate into concomitant errors in the stellar density and in the stellar radius and age. In order to overestimate the size of the planets, the impact parameter would need to have been overestimated. The distribution of measured planetary impact parameters, however, shows no evidence for this being the case (Figure 17). The impact parameter for the WASP planets appears to be uniformly distributed between 0 and 1 , as expected for random orbital orientations, whereas the HAT planets are, if anything, biased toward low impact parameters (if these are in error, the stars and planets would be even larger than currently estimated).

Stellar Atmospheric Parameters: Other potential sources of systematic errors include errors in the stellar effective temperatures (if the stars are hotter than measured, they would be closer to the ZAMS) or metallicities, or an error in the assumed stellar abundance pattern (generally stars are modeled assuming solar-scaled abundances). A check on the 
Table 6

Orbital and Planetary Parameters for HAT-P-65b and HAT-P-66b ${ }^{\mathrm{a}}$

\begin{tabular}{|c|c|c|}
\hline Parameter & $\begin{array}{l}\text { HAT-P-65b } \\
\text { Value }\end{array}$ & $\begin{array}{l}\text { HAT-P-66b } \\
\text { Value }\end{array}$ \\
\hline \multicolumn{3}{|l|}{ Light-curve parameters } \\
\hline$P$ (days) & $2.6054552 \pm 0.0000031$ & $2.9720860 \pm 0.0000057$ \\
\hline$T_{c}(\mathrm{BJD})^{\mathrm{b}}$ & $2456409.33263 \pm 0.00046$ & $2457258.79907 \pm 0.00072$ \\
\hline$T_{14}$ (days) $^{\mathrm{b}}$ & $0.1819 \pm 0.0022$ & $0.1958 \pm 0.0028$ \\
\hline$T_{12}=T_{34}(\text { days })^{\mathrm{b}}$ & $0.0215 \pm 0.0023$ & $0.0174 \pm 0.0025$ \\
\hline$a / R_{\star}$ & $4.57 \pm 0.20$ & $5.01_{-0.32}^{+0.21}$ \\
\hline$\zeta / R_{\star}{ }^{\mathrm{c}}$ & $12.438 \pm 0.080$ & $11.226 \pm 0.096$ \\
\hline$R_{p} / R_{\star}$ & $0.1045 \pm 0.0024$ & $0.0872 \pm 0.0024$ \\
\hline$b^{2}$ & $0.215_{-0.079}^{+0.070}$ & $0.110_{-0.081}^{+0.106}$ \\
\hline$b \equiv a \cos i / R_{\star}$ & $0.464_{-0.094}^{+0.070}$ & $0.33_{-0.16}^{+0.13}$ \\
\hline$i(\mathrm{deg})$ & $84.2 \pm 1.3$ & $86.2 \pm 1.8$ \\
\hline \multicolumn{3}{|l|}{ Limb-darkening coefficients ${ }^{\mathrm{d}}$} \\
\hline$c_{1}, r$ & 0.3439 & 0.3077 \\
\hline$c_{2}, r$ & 0.3359 & 0.3559 \\
\hline$c_{1}, i$ & 0.2544 & 0.2249 \\
\hline$c_{2}, i$ & 0.3414 & 0.3551 \\
\hline$c_{1}, z$ & 0.1949 & 0.1703 \\
\hline$c_{2}, z$ & 0.3379 & 0.3491 \\
\hline \multicolumn{3}{|l|}{ RV parameters } \\
\hline$K\left(\mathrm{~m} \mathrm{~s}^{-1}\right)$ & $68 \pm 11$ & $93.5 \pm 5.7$ \\
\hline$e^{\mathrm{e}}$ & $<0.304$ & $<0.090$ \\
\hline RV jitter HIRES $\left(\mathrm{m} \mathrm{s}^{-1}\right)^{\mathrm{f}}$ & $26.0 \pm 7.1$ & $<43.2$ \\
\hline RV jitter TRES $\left(\mathrm{m} \mathrm{s}^{-1}\right)$ & $\ldots$ & $<22.1$ \\
\hline RV jitter SOPHIE $\left(\mathrm{m} \mathrm{s}^{-1}\right)$ & $\ldots$ & $<16.3$ \\
\hline \multicolumn{3}{|l|}{ Planetary parameters } \\
\hline$M_{p}\left(M_{\mathrm{J}}\right)$ & $0.527 \pm 0.083$ & $0.783 \pm 0.057$ \\
\hline$R_{p}\left(R_{\mathrm{J}}\right)$ & $1.89 \pm 0.13$ & $1.59_{-0.10}^{+0.16}$ \\
\hline$C\left(M_{p}, R_{p}\right)^{\mathrm{g}}$ & 0.10 & 0.29 \\
\hline$\rho_{p}\left(\mathrm{~g} \mathrm{~cm}^{-3}\right)$ & $0.096 \pm 0.025$ & $0.242_{-0.061}^{+0.045}$ \\
\hline $\log g_{p}(\mathrm{cgs})$ & $2.560 \pm 0.090$ & $2.884_{-0.081}^{+0.051}$ \\
\hline$a(\mathrm{au})$ & $0.03951 \pm 0.00054$ & $0.04363_{-0.00064}^{+0.00121}$ \\
\hline$T_{\text {eq }}(\mathrm{K})$ & $1930 \pm 45$ & $1896_{-42}^{+66}$ \\
\hline$\Theta^{\mathrm{h}}$ & $0.0180 \pm 0.0031$ & $0.0336 \pm 0.0034$ \\
\hline $\log _{10}\langle F\rangle(\operatorname{cgs})^{\mathrm{i}}$ & $9.495 \pm 0.041$ & $9.465_{-0.039}^{+0.060}$ \\
\hline
\end{tabular}

Notes. For both systems the fixed-circular-orbit model has a higher Bayesian evidence than the eccentric-orbit model. We therefore assume a fixed circular orbit in generating the parameters listed here.

${ }^{\text {a }}$ We adopt the IAU 2015 Resolution B3 nominal values for the solar and Jovian parameters (Prša et al. 2016) for all of our calculations, taking $R_{\mathrm{J}}$ to be the nominal equatorial radius of Jupiter. Where necessary we assume $G=6.6408 \times 10^{-11} \mathrm{~m}^{3} \mathrm{~kg}^{-1} \mathrm{~s}^{-1}$. Because Yi et al. (2001) do not specify the assumed value for $G$ or $M_{\odot}$, we take the stellar masses from these isochrones at face value without conversion. Any discrepancy results in an error that is less than $1 \%$, which is well below the observational uncertainty. We note that the standard values assumed in prior HAT planet discovery papers are very close to the nominal values adopted here. In all cases the conversion results in changes to measured parameters that are indetectable at the level of precision to which they are listed.

${ }^{\mathrm{b}}$ Times are in Barycentric Julian Date calculated directly from UTC without correction for leap seconds. $T_{c}$ : reference epoch of mid-transit that minimizes the correlation with the orbital period. $T_{14}$ : total transit duration, time between first to last contact; $T_{12}=T_{34}$ : ingress/egress time, time between first and second or third and fourth contact.

${ }^{\mathrm{c}}$ Reciprocal of the half duration of the transit used as a jump parameter in our MCMC analysis in place of $a / R_{\star}$. It is related to $a / R_{\star}$ by the expression $\zeta / R_{\star}=a / R_{\star}(2 \pi(1+e \sin \omega)) /\left(P \sqrt{1-b^{2}} \sqrt{1-e^{2}}\right)$ (Bakos et al. 2010).

Values for a quadratic law, adopted from the tabulations by Claret (2004) according to the spectroscopic (SPC) parameters listed in Table 5.

e The 95\% confidence upper limit on the eccentricity determined when $\sqrt{e} \cos \omega$ and $\sqrt{e} \sin \omega$ are allowed to vary in the fit.

${ }^{\mathrm{f}}$ Term added in quadrature to the formal RV uncertainties for each instrument. This is treated as a free parameter in the fitting routine. In cases where the jitter is consistent with zero we list the $95 \%$ confidence upper limit.

${ }^{\mathrm{g}}$ Correlation coefficient between the planetary mass $M_{p}$ and radius $R_{p}$ estimated from the posterior parameter distribution.

${ }^{\mathrm{h}}$ The Safronov number is given by $\Theta=\frac{1}{2}\left(V_{\mathrm{esc}} / V_{\mathrm{orb}}\right)^{2}=\left(a / R_{p}\right)\left(M_{p} / M_{\star}\right)$ (see Hansen \& Barman 2007).

${ }^{\mathrm{i}}$ Incoming flux per unit surface area, averaged over the orbit.

spectroscopic temperature estimates can be performed by comparing the broadband photometric colors with the spectroscopically determined temperatures. We show this comparison in Figure 18, where we use the color of the points to show the planet radius. While there is perhaps a slight systematic difference in the $V-K$ versus $T_{\text {eff } \star}$ relation between large- and small-radius planets, with large-radius planets being found, on average, around slightly redder stars at fixed $T_{\text {eff } \star}$ than small- 
Table 7

Adopted Parameters for Transiting Planet Systems Discovered by HAT, KELT, TrES, and WASP

\begin{tabular}{|c|c|c|c|c|c|c|c|c|c|c|c|}
\hline Planet & $\begin{array}{l}\text { Period } \\
\text { (days) }\end{array}$ & $\begin{array}{c}M_{p} \\
\left(M_{\mathrm{J}}\right) \\
\end{array}$ & $\begin{array}{c}R_{p} \\
\left(R_{\mathrm{J}}\right) \\
\end{array}$ & $\begin{array}{l}T_{\mathrm{eq}} \\
(\mathrm{K})\end{array}$ & $\begin{array}{l}T_{\text {eff } \star} \\
(\mathrm{K})\end{array}$ & $\begin{array}{l}\rho_{\star} \\
\left(\mathrm{g} \mathrm{cm}^{-3}\right)\end{array}$ & {$[\mathrm{Fe} / \mathrm{H}]$} & $\begin{array}{c}M_{\star} \\
\left(M_{\odot}\right)\end{array}$ & $\begin{array}{l}\text { Age } \\
(\mathrm{Gyr})\end{array}$ & $\begin{array}{l}t_{\mathrm{tot}} \\
(\mathrm{Gyr})\end{array}$ & References \\
\hline HAT-P-10/ & 3.722 & $0.487 \pm 0.018$ & $1.005_{-0.027}^{+0.032}$ & $1020 \pm 17$ & $4980 \pm 60$ & $2.374_{-0.189}^{+0.208}$ & $0.13 \pm 0.08$ & $0.830 \pm 0.030$ & $7.90 \pm 3.80$ & 19.95 & 28 \\
\hline WASP-11b & $\ldots$ & $\ldots$ & $\ldots$ & $\ldots$ & $\ldots$ & $\ldots$ & $\cdots$ & $\ldots$ & $\ldots$ & $\cdots$ & $\cdots$ \\
\hline HAT-P-11b & 4.888 & $0.081 \pm 0.009$ & $0.422 \pm 0.014$ & $878 \pm 15$ & $4780 \pm 50$ & $2.699_{-0.222}^{+0.242}$ & $0.31 \pm 0.05$ & $0.810_{-0.030}^{+0.020}$ & $6.50_{-4.10}^{+5.90}$ & 19.95 & 45 \\
\hline HAT-P-12b & 3.213 & $0.211 \pm 0.012$ & $0.959_{-0.021}^{+0.029}$ & $963 \pm 16$ & $4650 \pm 60$ & $2.999_{-0.214}^{+0.175}$ & $-0.29 \pm 0.05$ & $0.733 \pm 0.018$ & $2.50 \pm 2.00$ & 19.95 & 32 \\
\hline HAT-P-13b & 2.916 & $0.850 \pm 0.038$ & $1.281 \pm 0.079$ & $1656_{-43}^{+46}$ & $5653 \pm 90$ & $0.448_{-0.071}^{+0.082}$ & $0.41 \pm 0.08$ & $1.220_{-0.100}^{+0.050}$ & $5.00_{-0.70}^{+2.50}$ & 6.80 & 34,51 \\
\hline HAT-P-14b & 4.628 & $2.232 \pm 0.059$ & $1.150 \pm 0.052$ & $1570 \pm 34$ & $6600 \pm 90$ & $0.618_{-0.066}^{+0.078}$ & $0.11 \pm 0.08$ & $1.386 \pm 0.045$ & $1.30 \pm 0.40$ & 4.20 & 50 \\
\hline HAT-P-15b & 10.864 & $1.946 \pm 0.066$ & $1.072 \pm 0.043$ & $904 \pm 20$ & $5568 \pm 90$ & $1.137_{-0.122}^{+0.142}$ & $0.22 \pm 0.08$ & $1.013 \pm 0.043$ & $6.80_{-1.60}^{+2.50}$ & 13.10 & 55 \\
\hline HAT-P-16b & 2.776 & $4.193 \pm 0.094$ & $1.289 \pm 0.066$ & $1626 \pm 40$ & $6158 \pm 80$ & $0.908_{-0.113}^{+0.134}$ & $0.17 \pm 0.08$ & $1.218 \pm 0.039$ & $2.00 \pm 0.80$ & 6.60 & 52 \\
\hline HAT-P-17b & 10.339 & $0.530 \pm 0.019$ & $1.000 \pm 0.030$ & $787 \pm 15$ & $5246 \pm 80$ & $2.123_{-0.188}^{+0.207}$ & $0.00 \pm 0.08$ & $0.861 \pm 0.039$ & $6.90 \pm 3.30$ & 19.95 & 97 \\
\hline HAT-P-18b & 5.508 & $0.197 \pm 0.013$ & $0.995 \pm 0.052$ & $852 \pm 28$ & $4803 \pm 80$ & $2.589_{-0.359}^{+0.432}$ & $0.10 \pm 0.08$ & $0.770 \pm 0.031$ & $12.40_{-6.40}^{+4.40}$ & 19.95 & 75 \\
\hline HAT-P-19b & 4.009 & $0.292 \pm 0.018$ & $1.132 \pm 0.072$ & $1010 \pm 42$ & $4989 \pm 126$ & $2.169_{-0.353}^{+0.438}$ & $0.23 \pm 0.08$ & $0.842 \pm 0.042$ & $8.80 \pm 5.20$ & 19.95 & 75 \\
\hline HAT-P-1b & 4.465 & $0.524 \pm 0.031$ & $1.225 \pm 0.059$ & $1306 \pm 30$ & $6076 \pm 27$ & $1.150_{-0.161}^{+0.188}$ & $0.21 \pm 0.03$ & $1.133_{-0.079}^{+0.075}$ & $2.70_{-2.00}^{+2.50}$ & 8.70 & $11,13,18$ \\
\hline HAT-P-20b & 2.875 & $7.246 \pm 0.187$ & $0.867 \pm 0.033$ & $970 \pm 23$ & $4595 \pm 80$ & $3.196_{-0.297}^{+0.336}$ & $0.35 \pm 0.08$ & $0.756 \pm 0.028$ & $6.70_{-3.80}^{+5.70}$ & 19.95 & 84 \\
\hline HAT-P-21b & 4.124 & $4.063 \pm 0.161$ & $1.024 \pm 0.092$ & $1283 \pm 50$ & $5588 \pm 80$ & $0.992_{-0.200}^{+0.260}$ & $0.01 \pm 0.08$ & $0.947 \pm 0.042$ & $10.20 \pm 2.50$ & 15.00 & 84 \\
\hline HAT-P-22b & 3.212 & $2.147 \pm 0.061$ & $1.080 \pm 0.058$ & $1283 \pm 32$ & $5302 \pm 80$ & $1.154_{-0.140}^{+0.164}$ & $0.24 \pm 0.08$ & $0.916 \pm 0.035$ & $12.40 \pm 2.60$ & 18.85 & 84 \\
\hline HAT-P-23b & 1.213 & $2.090 \pm 0.111$ & $1.368 \pm 0.090$ & $2056 \pm 66$ & $5905 \pm 80$ & $0.915_{-0.152}^{+0.189}$ & $0.15 \pm 0.04$ & $1.130 \pm 0.035$ & $4.00 \pm 1.00$ & 8.55 & 84 \\
\hline HAT-P-24b & 3.355 & $0.685 \pm 0.033$ & $1.242 \pm 0.067$ & $1637 \pm 42$ & $6373 \pm 80$ & $0.737_{-0.105}^{+0.129}$ & $-0.16 \pm 0.08$ & $1.191 \pm 0.042$ & $2.80 \pm 0.60$ & 5.95 & 56 \\
\hline HAT-P-25b & 3.653 & $0.567 \pm 0.022$ & $1.190_{-0.056}^{+0.081}$ & $1202 \pm 36$ & $5500 \pm 80$ & $1.616_{-0.247}^{+0.213}$ & $0.31 \pm 0.08$ & $1.010 \pm 0.032$ & $3.20 \pm 2.30$ & 13.50 & 96 \\
\hline HAT-P-26b & 4.235 & $0.059 \pm 0.007$ & $0.565_{-0.032}^{+0.072}$ & $1001_{-37}^{+66}$ & $5079 \pm 88$ & $2.351_{-0.714}^{+0.443}$ & $-0.04 \pm 0.08$ & $0.816 \pm 0.033$ & $9.00_{-4.90}^{+3.00}$ & 19.95 & 77 \\
\hline HAT-P-27b & 3.040 & $0.660 \pm 0.033$ & $1.038_{-0.058}^{+0.077}$ & $1207 \pm 41$ & $5302 \pm 88$ & $1.842_{-0.306}^{+0.269}$ & $0.29 \pm 0.10$ & $0.945 \pm 0.035$ & $4.40_{-2.60}^{+3.80}$ & 17.10 & 80 \\
\hline HAT-P-28b & 3.257 & $0.636 \pm 0.037$ & $1.189_{-0.075}^{+0.02}$ & $1371 \pm 50$ & $5681 \pm 88$ & $1.143_{-0.239}^{+0.233}$ & $0.12 \pm 0.08$ & $1.024 \pm 0.046$ & $5.80 \pm 2.30$ & 12.05 & 79 \\
\hline HAT-P-29b & 5.723 & $0.778_{-0.040}^{+0.076}$ & $1.107_{-0.082}^{+0.136}$ & $1260_{-45}^{+64}$ & $6087 \pm 88$ & $0.926_{-0.251}^{+0.201}$ & $0.21 \pm 0.08$ & $1.207 \pm 0.046$ & $2.20 \pm 1.00$ & 6.90 & 79 \\
\hline HAT-P-2b & 5.633 & $9.090 \pm 0.240$ & $1.157_{-0.062}^{+0.073}$ & $1540 \pm 30$ & $6290 \pm 60$ & $0.435_{-0.065}^{+0.073}$ & $0.14 \pm 0.08$ & $1.360 \pm 0.040$ & $2.60 \pm 0.50$ & 4.55 & 60 \\
\hline HAT-P-30b & 2.811 & $0.711 \pm 0.028$ & $1.340 \pm 0.065$ & $1630 \pm 42$ & $6304 \pm 88$ & $0.974_{-0.113}^{+0.137}$ & $0.13 \pm 0.08$ & $1.242 \pm 0.041$ & $1.00_{-0.50}^{+0.80}$ & 6.05 & 81 \\
\hline HAT-P-31b & 5.005 & $2.171_{-0.077}^{+0.105}$ & $1.070_{-0.320}^{+0.480}$ & $1450_{-110}^{+230}$ & $6065 \pm 100$ & $0.690_{-0.260}^{+0.340}$ & $0.15 \pm 0.08$ & $1.218_{-0.063}^{+0.089}$ & $3.17_{-1.11}^{+0.70}$ & 6.55 & 74 \\
\hline HAT-P-32b & 2.150 & $0.860 \pm 0.164$ & $1.789 \pm 0.025$ & $1786 \pm 26$ & $6207 \pm 88$ & $0.903_{-0.047}^{+0.050}$ & $-0.04 \pm 0.08$ & $1.160 \pm 0.041$ & $2.70 \pm 0.80$ & 7.00 & 85 \\
\hline HAT-P-33b & 3.474 & $0.762 \pm 0.101$ & $1.686 \pm 0.045$ & $1782 \pm 28$ & $6446 \pm 88$ & $0.442_{-0.030}^{+0.033}$ & $0.07 \pm 0.08$ & $1.375 \pm 0.040$ & $2.30 \pm 0.30$ & 4.25 & 85 \\
\hline HAT-P-34b & 5.453 & $3.328 \pm 0.211$ & $1.197_{-0.092}^{+0.128}$ & $1520 \pm 60$ & $6442 \pm 88$ & $0.542_{-0.122}^{+0.129}$ & $0.22 \pm 0.04$ & $1.392 \pm 0.047$ & $1.70_{-0.50}^{+0.40}$ & 4.35 & 95 \\
\hline HAT-P-35b & 3.647 & $1.054 \pm 0.033$ & $1.332 \pm 0.098$ & $1581 \pm 45$ & $6096 \pm 88$ & $0.590_{-0.098}^{+0.120}$ & $0.11 \pm 0.08$ & $1.236 \pm 0.048$ & $3.50_{-0.50}^{+0.80}$ & 6.10 & 95 \\
\hline HAT-P-36b & 1.327 & $1.832 \pm 0.099$ & $1.264 \pm 0.071$ & $1823 \pm 55$ & $5560 \pm 100$ & $1.099_{-0.159}^{+0.194}$ & $0.26 \pm 0.10$ & $1.022 \pm 0.049$ & $6.60_{-1.80}^{+2.90}$ & 12.80 & 95 \\
\hline HAT-P-37b & 2.797 & $1.169 \pm 0.103$ & $1.178 \pm 0.077$ & $1271 \pm 47$ & $5500 \pm 100$ & $1.942_{-0.351}^{+0.342}$ & $0.03 \pm 0.10$ & $0.929 \pm 0.043$ & $3.60_{-2.20}^{+4.10}$ & 16.25 & 95 \\
\hline HAT-P-38b & 4.640 & $0.267 \pm 0.020$ & $0.825_{-0.063}^{+0.092}$ & $1082 \pm 55$ & $5330 \pm 100$ & $1.588_{-0.415}^{+0.413}$ & $0.06 \pm 0.10$ & $0.886 \pm 0.044$ & $10.10 \pm 4.80$ & 19.50 & 104 \\
\hline HAT-P-39b & 3.544 & $0.599 \pm 0.099$ & $1.571_{-0.081}^{+0.108}$ & $1752 \pm 43$ & $6430 \pm 100$ & $0.461_{-0.063}^{+0.059}$ & $0.19 \pm 0.10$ & $1.404 \pm 0.051$ & $2.00 \pm 0.40$ & 4.20 & 94 \\
\hline HAT-P-3b & 2.900 & $0.596_{-0.026}^{+0.024}$ & $0.899_{-0.049}^{+0.043}$ & $1127_{-39}^{+49}$ & $5185 \pm 46$ & $2.254_{-0.281}^{+0.423}$ & $0.27 \pm 0.04$ & $0.928_{-0.054}^{+0.044}$ & $1.50_{-1.40}^{+5.40}$ & 18.15 & $3,11,59$ \\
\hline HAT-P-40b & 4.457 & $0.615 \pm 0.038$ & $1.730 \pm 0.062$ & $1770 \pm 33$ & $6080 \pm 100$ & $0.196_{-0.020}^{+0.020}$ & $0.22 \pm 0.10$ & $1.512_{-0.109}^{+0.045}$ & $2.70_{-0.30}^{+0.90}$ & 3.35 & 94 \\
\hline HAT-P-41b & 2.694 & $0.800 \pm 0.102$ & $1.685_{-0.051}^{+0.076}$ & $1941 \pm 38$ & $6390 \pm 100$ & $0.418_{-0.042}^{+0.033}$ & $0.21 \pm 0.10$ & $1.418 \pm 0.047$ & $2.20 \pm 0.40$ & 4.05 & 94 \\
\hline HAT-P-42b & 4.642 & $0.975 \pm 0.126$ & $1.277 \pm 0.149$ & $1427 \pm 58$ & $5743 \pm 50$ & $0.467_{-0.111}^{+0.154}$ & $0.27 \pm 0.08$ & $1.179 \pm 0.067$ & $5.10_{-0.70}^{+1.80}$ & 7.65 & 112 \\
\hline HAT-P-43b & 3.333 & $0.660 \pm 0.083$ & $1.283_{-0.034}^{+0.057}$ & $1361 \pm 24$ & $5645 \pm 74$ & $1.091_{-0.106}^{+0.083}$ & $0.23 \pm 0.08$ & $1.048_{-0.042}^{+0.031}$ & $5.70_{-1.10}^{+1.90}$ & 11.65 & 112 \\
\hline HAT-P-44b & 4.301 & $0.392 \pm 0.031$ & $1.280_{-0.074}^{+0.145}$ & $1126_{-42}^{+67}$ & $5295 \pm 100$ & $1.402_{-0.388}^{+0.277}$ & $0.33 \pm 0.10$ & $0.939 \pm 0.041$ & $8.90 \pm 3.90$ & 17.50 & 123 \\
\hline HAT-P-45b & 3.129 & $0.892_{-0.099}^{+0.137}$ & $1.426_{-0.087}^{+0.175}$ & $1652_{-52}^{+90}$ & $6330 \pm 100$ & $0.776_{-0.220}^{+0.149}$ & $0.07 \pm 0.10$ & $1.259 \pm 0.058$ & $2.00 \pm 0.80$ & 5.65 & 123 \\
\hline HAT-P-46b & 4.463 & $0.493_{-0.052}^{+0.082}$ & $1.284_{-0.133}^{+0.271}$ & $1458_{-75}^{+140}$ & $6120 \pm 100$ & $0.676_{-0.291}^{+0.249}$ & $0.30 \pm 0.10$ & $1.284_{-0.060}^{+0.095}$ & $2.50_{-1.00}^{+0.70}$ & 5.75 & 123 \\
\hline HAT-P-47b & 4.732 & $0.206 \pm 0.039$ & $1.313 \pm 0.045$ & $1605 \pm 22$ & $6703 \pm 50$ & $0.564_{-0.045}^{+0.049}$ & $0.00 \pm 0.08$ & $1.387 \pm 0.038$ & $1.50 \pm 0.30$ & 3.95 & 161 \\
\hline
\end{tabular}


Table 7

(Continued)

\begin{tabular}{|c|c|c|c|c|c|c|c|c|c|c|c|}
\hline Planet & $\begin{array}{l}\text { Period } \\
\text { (days) }\end{array}$ & $\begin{array}{c}M_{p} \\
\left(M_{\mathrm{J}}\right)\end{array}$ & $\begin{array}{c}R_{p} \\
\left(R_{\mathrm{J}}\right)\end{array}$ & $\begin{array}{l}T_{\mathrm{eq}} \\
(\mathrm{K})\end{array}$ & $\begin{array}{c}T_{\text {eff } \star} \\
(\mathrm{K})\end{array}$ & $\begin{array}{l}\rho_{\star} \\
\left(\mathrm{g} \mathrm{cm}^{-3}\right)\end{array}$ & {$[\mathrm{Fe} / \mathrm{H}]$} & $\begin{array}{c}M_{\star} \\
\left(M_{\odot}\right)\end{array}$ & $\begin{array}{l}\text { Age } \\
\text { (Gyr) }\end{array}$ & $\begin{array}{c}t_{\text {tot }} \\
(\mathrm{Gyr})\end{array}$ & References \\
\hline HAT-P-48b & 4.409 & $0.168 \pm 0.024$ & $1.131 \pm 0.054$ & $1361 \pm 25$ & $5946 \pm 50$ & $0.847_{-0.093}^{+0.109}$ & $0.02 \pm 0.08$ & $1.099 \pm 0.041$ & $4.70_{-0.80}^{+1.30}$ & 8.80 & 161 \\
\hline HAT-P-49b & 2.692 & $1.730 \pm 0.205$ & $1.413_{-0.077}^{+0.128}$ & $2131_{-42}^{+69}$ & $6820 \pm 52$ & $0.353_{-0.069}^{+0.049}$ & $0.07 \pm 0.08$ & $1.543 \pm 0.051$ & $1.50 \pm 0.20$ & 2.95 & 126 \\
\hline HAT-P-4b & 3.057 & $0.556 \pm 0.068$ & $1.367_{-0.044}^{+0.052}$ & $1686_{-26}^{+30}$ & $5860 \pm 80$ & $0.427_{-0.058}^{+0.073}$ & $0.24 \pm 0.08$ & $1.248_{-0.120}^{+0.070}$ & $4.60_{-1.00}^{+2.20}$ & 6.20 & $5,11,71$ \\
\hline HAT-P-50b & 3.122 & $1.350 \pm 0.073$ & $1.288 \pm 0.064$ & $1862 \pm 34$ & $6280 \pm 49$ & $0.357 \pm 0.037$ & $-0.18 \pm 0.08$ & $1.273_{-0.115}^{+0.049}$ & $3.37_{-0.27}^{+1.44}$ & 4.75 & 135 \\
\hline HAT-P-51b & 4.218 & $0.309 \pm 0.018$ & $1.293 \pm 0.054$ & $1192 \pm 21$ & $5449 \pm 50$ & $1.223_{-0.135}^{+0.100}$ & $0.27 \pm 0.08$ & $0.976 \pm 0.028$ & $8.20 \pm 1.70$ & 15.15 & 135 \\
\hline HAT-P-52b & 2.754 & $0.818 \pm 0.029$ & $1.009 \pm 0.072$ & $1218 \pm 37$ & $5131 \pm 50$ & $1.750 \pm 0.290$ & $0.28 \pm 0.08$ & $0.887 \pm 0.027$ & $9.40 \pm 4.10$ & 19.95 & 135 \\
\hline HAT-P-53b & 1.962 & $1.484 \pm 0.056$ & $1.318 \pm 0.091$ & $1778 \pm 48$ & $5956 \pm 50$ & $0.870 \pm 0.130$ & $0.00 \pm 0.08$ & $1.093 \pm 0.043$ & $4.67_{-0.83}^{+1.45}$ & 8.90 & 135 \\
\hline HAT-P-54b & 3.800 & $0.760 \pm 0.032$ & $0.944 \pm 0.028$ & $818 \pm 12$ & $4390 \pm 50$ & $3.876_{-0.253}^{+0.283}$ & $-0.13 \pm 0.08$ & $0.645 \pm 0.020$ & $3.90_{-2.10}^{+4.30}$ & 19.95 & 132 \\
\hline HAT-P-55b & 3.585 & $0.582 \pm 0.056$ & $1.182 \pm 0.055$ & $1313 \pm 26$ & $5808 \pm 50$ & $1.380_{-0.143}^{+0.167}$ & $-0.03 \pm 0.08$ & $1.013 \pm 0.037$ & $4.20 \pm 1.70$ & 11.50 & 151 \\
\hline HAT-P-56b & 2.791 & $2.180 \pm 0.250$ & $1.466 \pm 0.040$ & $1840 \pm 21$ & $6566 \pm 50$ & $0.627 \pm 0.033$ & $-0.08 \pm 0.08$ & $1.296 \pm 0.036$ & $2.01 \pm 0.35$ & 4.75 & 140 \\
\hline HAT-P-57b & 2.465 & $0.000_{-0.000}^{+1.850}$ & $1.413 \pm 0.054$ & $2200 \pm 76$ & $7500 \pm 250$ & $0.615_{-0.036}^{+0.022}$ & $-0.25 \pm 0.25$ & $1.470 \pm 0.120$ & $1.00_{-0.51}^{+0.67}$ & 2.85 & 137 \\
\hline HAT-P-58b & 4.014 & $0.394 \pm 0.034$ & $1.203_{-0.063}^{+0.090}$ & $1500_{-29}^{+49}$ & $5931 \pm 50$ & $0.564 \pm 0.066$ & $0.01 \pm 0.08$ & $1.110_{-0.042}^{+0.080}$ & $6.10_{-1.66}^{+0.82}$ & 8.45 & 162 \\
\hline HAT-P-59b & 4.142 & $1.624 \pm 0.061$ & $1.121 \pm 0.066$ & $1273 \pm 27$ & $5665 \pm 50$ & $1.070 \pm 0.130$ & $0.41 \pm 0.08$ & $1.089 \pm 0.022$ & $4.30 \pm 1.00$ & 10.20 & 162 \\
\hline HAT-P-5b & 2.788 & $1.060 \pm 0.110$ & $1.254_{-0.056}^{+0.051}$ & $1539_{-32}^{+33}$ & $5960 \pm 100$ & $1.019_{-0.129}^{+0.162}$ & $0.24 \pm 0.15$ & $1.157_{-0.081}^{+0.043}$ & $2.60_{-1.40}^{+2.10}$ & 8.10 & 6,11 \\
\hline HAT-P-60b & 4.795 & $0.492 \pm 0.049$ & $1.116_{-0.064}^{+0.150}$ & $1662_{-42}^{+73}$ & $6462 \pm 50$ & $0.357_{-0.082}^{+0.055}$ & $-0.24 \pm 0.08$ & $1.310 \pm 0.070$ & $2.88 \pm 0.56$ & 4.10 & 162 \\
\hline HAT-P-61b & 1.902 & $1.103_{-0.071}^{+0.052}$ & $0.968_{-0.047}^{+0.061}$ & $1526 \pm 36$ & $5551 \pm 50$ & $1.510 \pm 0.180$ & $0.40 \pm 0.08$ & $1.043 \pm 0.022$ & $2.70_{-2.50}^{+1.80}$ & 11.95 & 162 \\
\hline HAT-P-62b & 2.645 & $0.801 \pm 0.088$ & $1.131_{-0.053}^{+0.074}$ & $1523 \pm 31$ & $5601 \pm 50$ & $0.840 \pm 0.087$ & $0.45 \pm 0.08$ & $1.103 \pm 0.028$ & $5.40 \pm 0.89$ & 9.60 & 162 \\
\hline HAT-P-63b & 3.378 & $0.638 \pm 0.023$ & $1.213 \pm 0.094$ & $1246 \pm 32$ & $5365 \pm 50$ & $1.320 \pm 0.180$ & $0.43 \pm 0.08$ & $0.976 \pm 0.022$ & $7.20 \pm 2.00$ & 14.95 & 162 \\
\hline HAT-P-64b & 4.007 & $0.750 \pm 0.160$ & $1.790 \pm 0.140$ & $1741_{-35}^{+48}$ & $6302 \pm 50$ & $0.332_{-0.054}^{+0.040}$ & $-0.01 \pm 0.08$ & $1.369 \pm 0.046$ & $2.87 \pm 0.30$ & 4.10 & 162 \\
\hline HAT-P-65b & 2.605 & $0.527 \pm 0.083$ & $1.890 \pm 0.130$ & $1930 \pm 45$ & $5835 \pm 51$ & $0.266 \pm 0.036$ & $0.10 \pm 0.08$ & $1.212 \pm 0.050$ & $5.46 \pm 0.61$ & 6.50 & 166 \\
\hline HAT-P-66b & 2.972 & $0.783 \pm 0.057$ & $1.590_{-0.100}^{+0.160}$ & $1897_{-42}^{+66}$ & $6002 \pm 50$ & $0.269 \pm 0.040$ & $0.04 \pm 0.08$ & $1.255_{-0.054}^{+0.107}$ & $4.66_{-1.12}^{+0.52}$ & 5.60 & 166 \\
\hline HAT-P-6b & 3.853 & $1.059_{-0.052}^{+0.053}$ & $1.330_{-0.058}^{+0.064}$ & $1675_{-31}^{+32}$ & $6570 \pm 80$ & $0.581_{-0.080}^{+0.088}$ & $-0.13 \pm 0.08$ & $1.290_{-0.066}^{+0.064}$ & $2.30_{-0.60}^{+0.50}$ & 4.65 & $10,11,58$ \\
\hline HAT-P-7b & 2.205 & $1.820 \pm 0.030$ & $1.500 \pm 0.020$ & $2140_{-60}^{+110}$ & $6350 \pm 80$ & $0.271 \pm 0.003$ & $0.26 \pm 0.08$ & $1.530 \pm 0.040$ & $2.14 \pm 0.26$ & 3.25 & $12,46,47,48$ \\
\hline HAT-P-8b & 3.076 & $1.520_{-0.160}^{+0.180}$ & $1.500_{-0.060}^{+0.080}$ & $1700 \pm 35$ & $6200 \pm 80$ & $0.458_{-0.063}^{+0.058}$ & $0.01 \pm 0.08$ & $1.280 \pm 0.040$ & $3.40 \pm 1.00$ & 5.20 & 31 \\
\hline HAT-P-9b & 3.923 & $0.780 \pm 0.090$ & $1.400 \pm 0.060$ & $1530 \pm 40$ & $6350 \pm 150$ & $0.782_{-0.135}^{+0.166}$ & $0.12 \pm 0.20$ & $1.280 \pm 0.130$ & $1.60_{-1.40}^{+1.80}$ & 5.50 & 23,103 \\
\hline HATS-10b & 3.313 & $0.526 \pm 0.081$ & $0.969_{-0.045}^{+0.061}$ & $1407 \pm 39$ & $5880 \pm 120$ & $1.150_{-0.160}^{+0.120}$ & $0.15 \pm 0.10$ & $1.101 \pm 0.054$ & $3.30 \pm 1.70$ & 9.40 & 138 \\
\hline HATS-11b & 3.619 & $0.850 \pm 0.120$ & $1.510 \pm 0.078$ & $1637 \pm 48$ & $6060 \pm 150$ & $0.471_{-0.052}^{+0.037}$ & $-0.39 \pm 0.06$ & $1.000 \pm 0.060$ & $7.70_{-1.60}^{+2.20}$ & 9.40 & 156 \\
\hline HATS-12b & 3.143 & $2.380 \pm 0.110$ & $1.350 \pm 0.170$ & $2097 \pm 89$ & $6408 \pm 75$ & $0.196_{-0.044}^{+0.057}$ & $-0.10 \pm 0.04$ & $1.489 \pm 0.071$ & $2.36 \pm 0.31$ & 3.00 & 156 \\
\hline HATS-13b & 3.044 & $0.543 \pm 0.072$ & $1.212 \pm 0.035$ & $1244 \pm 20$ & $5523 \pm 69$ & $1.930 \pm 0.110$ & $0.05 \pm 0.06$ & $0.962 \pm 0.029$ & $2.50 \pm 1.70$ & 14.55 & 131 \\
\hline HATS-14b & 2.767 & $1.071 \pm 0.070$ & $1.039_{-0.022}^{+0.032}$ & $1276 \pm 20$ & $5408 \pm 65$ & $1.682_{-0.126}^{+0.071}$ & $0.28 \pm 0.03$ & $0.967 \pm 0.024$ & $4.90 \pm 1.70$ & 15.70 & 131,145 \\
\hline HATS-15b & 1.747 & $2.170 \pm 0.150$ & $1.105 \pm 0.040$ & $1505 \pm 30$ & $5311 \pm 77$ & $1.570 \pm 0.120$ & $0.00 \pm 0.05$ & $0.871 \pm 0.023$ & $11.00_{-2.00}^{+1.40}$ & 19.95 & 150 \\
\hline HATS-16b & 2.687 & $3.270 \pm 0.190$ & $1.300 \pm 0.150$ & $1592_{-82}^{+61}$ & $5738 \pm 79$ & $0.720_{-0.130}^{+0.260}$ & $0.10 \pm 0.05$ & $0.970 \pm 0.035$ & $9.50 \pm 1.80$ & 14.50 & 150 \\
\hline HATS-17b & 16.255 & $1.338 \pm 0.065$ & $0.777 \pm 0.056$ & $814 \pm 25$ & $5846 \pm 78$ & $1.380 \pm 0.270$ & $0.30 \pm 0.03$ & $1.131 \pm 0.030$ & $2.10 \pm 1.30$ & 8.95 & 154 \\
\hline HATS-18b & 0.838 & $1.980 \pm 0.077$ & $1.337_{-0.049}^{+0.102}$ & $2060 \pm 59$ & $5600 \pm 120$ & $1.380_{-0.210}^{+0.130}$ & $0.28 \pm 0.08$ & $1.037 \pm 0.047$ & $4.20 \pm 2.20$ & 12.25 & 167 \\
\hline HATS-19b & 4.570 & $0.427 \pm 0.071$ & $1.660_{-0.210}^{+0.0270}$ & $1570 \pm 110$ & $5896 \pm 77$ & $0.340_{-0.110}^{+0.150}$ & $0.24 \pm 0.05$ & $1.303 \pm 0.083$ & $3.94_{-0.50}^{+0.96}$ & 5.40 & 164 \\
\hline HATS- $1 b$ & 3.446 & $1.855_{-0.196}^{+0.262}$ & $1.302_{-0.098}^{+0.162}$ & $1359_{-59}^{+89}$ & $5870 \pm 100$ & $1.241_{-0.370}^{+0.317}$ & $-0.06 \pm 0.12$ & $0.986 \pm 0.054$ & $6.00 \pm 2.80$ & 12.50 & 114 \\
\hline HATS-20b & 3.799 & $0.273 \pm 0.035$ & $0.776 \pm 0.055$ & $1147 \pm 36$ & $5406 \pm 49$ & $1.980 \pm 0.480$ & $0.03 \pm 0.05$ & $0.910 \pm 0.026$ & $6.40 \pm 3.40$ & 17.50 & 164 \\
\hline HATS-21b & 3.554 & $0.332_{-0.030}^{+0.040}$ & $1.123_{-0.054}^{+0.147}$ & $1284_{-31}^{+55}$ & $5695 \pm 67$ & $1.550 \pm 0.380$ & $0.30 \pm 0.04$ & $1.080 \pm 0.026$ & $2.30 \pm 1.70$ & 10.60 & 164 \\
\hline HATS-22b & 4.723 & $2.740 \pm 0.110$ & $0.953_{-0.029}^{+0.048}$ & $858_{-17}^{+24}$ & $4803 \pm 55$ & $3.260 \pm 0.680$ & $0.00 \pm 0.04$ & $0.759 \pm 0.019$ & $4.60_{-4.00}^{+5.80}$ & 19.95 & 163 \\
\hline HATS-23b & 2.161 & $1.470 \pm 0.072$ & $1.860_{-0.400}^{+0.300}$ & $1654 \pm 54$ & $5780 \pm 120$ & $0.920_{-0.110}^{+0.200}$ & $0.28 \pm 0.07$ & $1.121 \pm 0.046$ & $4.20 \pm 1.50$ & 9.20 & 163 \\
\hline HATS-24b & 1.348 & $2.440 \pm 0.180$ & $1.487_{-0.054}^{+0.078}$ & $2067 \pm 39$ & $6346 \pm 81$ & $1.096_{-0.085}^{+0.059}$ & $0.00 \pm 0.05$ & $1.212 \pm 0.033$ & $0.88_{-0.45}^{+0.67}$ & 6.15 & 163 \\
\hline HATS-25b & 4.299 & $0.613 \pm 0.042$ & $1.260 \pm 0.100$ & $1277 \pm 42$ & $5715 \pm 73$ & $1.030 \pm 0.200$ & $0.02 \pm 0.05$ & $0.994 \pm 0.035$ & $7.50 \pm 1.90$ & 12.70 & 159 \\
\hline HATS-26b & 3.302 & $0.650 \pm 0.076$ & $1.750 \pm 0.210$ & $1918 \pm 61$ & $6071 \pm 81$ & $0.219 \pm 0.033$ & $-0.02 \pm 0.05$ & $1.299_{-0.056}^{+0.113}$ & $4.04_{-0.94}^{+0.62}$ & 4.85 & 159 \\
\hline
\end{tabular}


Table 7

(Continued)

\begin{tabular}{|c|c|c|c|c|c|c|c|c|c|c|c|}
\hline Planet & $\begin{array}{l}\text { Period } \\
\text { (days) }\end{array}$ & $\begin{array}{c}M_{p} \\
\left(M_{\mathrm{J}}\right)\end{array}$ & $\begin{array}{c}R_{p} \\
\left(R_{\mathrm{J}}\right)\end{array}$ & $\begin{array}{l}T_{\mathrm{eq}} \\
(\mathrm{K})\end{array}$ & $\begin{array}{l}T_{\text {eff } \star} \\
(\mathrm{K})\end{array}$ & $\begin{array}{l}\rho_{\star} \\
\left(\mathrm{g} \mathrm{cm}^{-3}\right)\end{array}$ & {$[\mathrm{Fe} / \mathrm{H}]$} & $\begin{array}{c}M_{\star} \\
\left(M_{\odot}\right)\end{array}$ & $\begin{array}{l}\text { Age } \\
\text { (Gyr) }\end{array}$ & $\begin{array}{l}t_{\mathrm{tot}} \\
(\mathrm{Gyr})\end{array}$ & References \\
\hline HATS-27b & 4.637 & $0.540 \pm 0.130$ & $1.520 \pm 0.140$ & $1661 \pm 50$ & $6438 \pm 64$ & $0.370 \pm 0.059$ & $0.09 \pm 0.04$ & $1.415 \pm 0.045$ & $2.31 \pm 0.21$ & 3.90 & 159 \\
\hline HATS-28b & 3.181 & $0.672 \pm 0.087$ & $1.194 \pm 0.070$ & $1253 \pm 35$ & $5498 \pm 84$ & $1.680 \pm 0.270$ & $0.01 \pm 0.06$ & $0.929 \pm 0.036$ & $6.20 \pm 2.80$ & 16.10 & 159 \\
\hline HATS-29b & 4.606 & $0.653 \pm 0.063$ & $1.251 \pm 0.061$ & $1212 \pm 30$ & $5670 \pm 110$ & $1.170 \pm 0.110$ & $0.16 \pm 0.08$ & $1.032 \pm 0.049$ & $5.50_{-1.70}^{+2.60}$ & 11.95 & 159 \\
\hline HATS-2b & 1.354 & $1.345 \pm 0.150$ & $1.168 \pm 0.030$ & $1577 \pm 31$ & $5227 \pm 95$ & $1.718_{-0.127}^{+0.133}$ & $0.15 \pm 0.05$ & $0.882 \pm 0.037$ & $9.70 \pm 2.90$ & 19.95 & 111 \\
\hline HATS-30b & 3.174 & $0.706 \pm 0.039$ & $1.175 \pm 0.052$ & $1414 \pm 32$ & $5943 \pm 70$ & $1.340 \pm 0.190$ & $0.06 \pm 0.05$ & $1.093 \pm 0.031$ & $2.30 \pm 1.20$ & 9.20 & 159 \\
\hline HATS-31b & 3.378 & $0.880 \pm 0.120$ & $1.640 \pm 0.220$ & $1823 \pm 81$ & $6050 \pm 120$ & $0.275_{-0.061}^{+0.082}$ & $0.00 \pm 0.07$ & $1.275 \pm 0.096$ & $4.30 \pm 1.10$ & 5.25 & 165 \\
\hline HATS-32b & 2.813 & $0.920 \pm 0.100$ & $1.249_{-0.096}^{+0.144}$ & $1437 \pm 58$ & $5700 \pm 110$ & $1.190 \pm 0.230$ & $0.39 \pm 0.05$ & $1.099 \pm 0.044$ & $3.50 \pm 1.80$ & 9.90 & 165 \\
\hline HATS-33b & 2.550 & $1.192 \pm 0.053$ & $1.230_{-0.081}^{+0.112}$ & $1429 \pm 38$ & $5659 \pm 85$ & $1.420 \pm 0.170$ & $0.29 \pm 0.05$ & $1.062 \pm 0.032$ & $3.00 \pm 1.70$ & 11.25 & 165 \\
\hline HATS-34b & 2.106 & $0.941 \pm 0.072$ & $1.430 \pm 0.190$ & $1445 \pm 42$ & $5380 \pm 73$ & $1.440 \pm 0.250$ & $0.25 \pm 0.07$ & $0.955 \pm 0.031$ & $7.70 \pm 2.70$ & 16.30 & 165 \\
\hline HATS-35b & 1.821 & $1.266 \pm 0.077$ & $1.570_{-0.130}^{+0.170}$ & $2100 \pm 100$ & $6300 \pm 100$ & $0.530 \pm 0.180$ & $0.21 \pm 0.06$ & $1.347 \pm 0.060$ & $2.29 \pm 0.55$ & 4.85 & 165 \\
\hline HATS-3b & 3.548 & $1.071 \pm 0.136$ & $1.381 \pm 0.035$ & $1648 \pm 24$ & $6351 \pm 76$ & $0.617_{-0.041}^{+0.046}$ & $-0.16 \pm 0.07$ & $1.209 \pm 0.036$ & $3.20_{-0.40}^{+0.60}$ & 5.70 & 115 \\
\hline HATS- $4 \mathrm{~b}$ & 2.517 & $1.323 \pm 0.028$ & $1.020 \pm 0.037$ & $1315 \pm 21$ & $5403 \pm 50$ & $1.784_{-0.122}^{+0.143}$ & $0.43 \pm 0.08$ & $1.001 \pm 0.020$ & $2.10 \pm 1.60$ & 13.65 & 127 \\
\hline HATS-5b & 4.763 & $0.237 \pm 0.012$ & $0.912 \pm 0.025$ & $1025 \pm 17$ & $5304 \pm 50$ & $2.001_{-0.165}^{+0.180}$ & $0.19 \pm 0.08$ & $0.936 \pm 0.028$ & $3.60_{-1.90}^{+2.60}$ & 17.25 & 124 \\
\hline HATS-6b & 3.325 & $0.319 \pm 0.070$ & $0.998 \pm 0.019$ & $712 \pm 5$ & $3770 \pm 100$ & $4.360 \pm 0.150$ & $0.20 \pm 0.09$ & $0.574_{-0.027}^{+0.020}$ & $0.00 \pm 0.00$ & 19.95 & 133 \\
\hline HATS-7b & 3.185 & $0.120 \pm 0.012$ & $0.563_{-0.034}^{+0.046}$ & $1084 \pm 32$ & $4985 \pm 50$ & $2.206_{-0.356}^{+0.326}$ & $0.25 \pm 0.08$ & $0.849 \pm 0.027$ & $7.80 \pm 5.00$ & 19.95 & 143 \\
\hline HATS- $8 b$ & 3.584 & $0.138 \pm 0.019$ & $0.873_{-0.075}^{+0.123}$ & $1324_{-38}^{+79}$ & $5679 \pm 50$ & $1.159_{-0.373}^{+0.220}$ & $0.21 \pm 0.08$ & $1.056 \pm 0.037$ & $5.10 \pm 1.70$ & 11.25 & 139 \\
\hline HATS- $9 b$ & 1.915 & $0.837 \pm 0.029$ & $1.065 \pm 0.098$ & $1823_{-35}^{+50}$ & $5366 \pm 70$ & $0.427_{-0.070}^{+0.030}$ & $0.34 \pm 0.05$ & $1.030 \pm 0.039$ & $10.80 \pm 1.50$ & 12.55 & 138 \\
\hline KELT-10b & 4.166 & $0.679_{-0.038}^{+0.039}$ & $1.399_{-0.049}^{+0.069}$ & $1377_{-23}^{+28}$ & $5948 \pm 74$ & $0.889_{-0.088}^{+0.062}$ & $0.09_{-0.10}^{+0.11}$ & $1.112_{-0.061}^{+0.055}$ & $3.20_{-0.51}^{+0.37}$ & 7.50 & 148 \\
\hline KELT-15b & 3.329 & $0.910_{-0.220}^{+0.210}$ & $1.443_{-0.057}^{+0.1110}$ & $1642_{-25}^{+45}$ & $6003_{-52}^{+56}$ & $0.514_{-0.076}^{+0.034}$ & $0.05 \pm 0.03$ & $1.181_{-0.050}^{+0.051}$ & $3.89_{-0.19}^{+0.18}$ & 5.95 & 149 \\
\hline KELT-1b & 1.218 & $27.230_{-0.480}^{+0.500}$ & $1.110_{-0.022}^{+0.032}$ & $2422_{-26}^{+32}$ & $6518 \pm 50$ & $0.597_{-0.039}^{+0.026}$ & $0.01 \pm 0.07$ & $1.324 \pm 0.026$ & $1.66_{-0.17}^{+0.18}$ & 4.30 & 99 \\
\hline KELT-2Ab & 4.114 & $1.486 \pm 0.088$ & $1.306_{-0.067}^{+0.081}$ & $1716_{-33}^{+39}$ & $6148_{-49}^{+48}$ & $0.296_{-0.041}^{+0.039}$ & $-0.01 \pm 0.07$ & $1.310_{-0.029}^{+0.032}$ & $3.08_{-0.12}^{+0.09}$ & 3.95 & 98 \\
\hline KELT-3b & 2.703 & $1.462_{-0.066}^{+0.067}$ & $1.358_{-0.069}^{+0.068}$ & $1821_{-37}^{+35}$ & $6304 \pm 49$ & $0.556_{-0.054}^{+0.065}$ & $0.05_{-0.08}^{+0.08}$ & $1.282_{-0.060}^{+0.062}$ & $2.50_{-0.26}^{+0.14}$ & 4.90 & 116 \\
\hline KELT-4Ab & 2.990 & $0.902_{-0.059}^{+0.060}$ & $1.699_{-0.045}^{+0.046}$ & $1823 \pm 27$ & $6206 \pm 75$ & $0.411_{-0.017}^{+0.018}$ & $-0.12_{-0.07}^{+0.07}$ & $1.201_{-0.061}^{+0.067}$ & $3.21_{-0.17}^{+0.13}$ & 4.65 & 153 \\
\hline KELT-6b & 7.846 & $0.430_{-0.046}^{+0.045}$ & $1.193_{-0.077}^{+0.130}$ & $1313_{-38}^{+59}$ & $6102 \pm 43$ & $0.387_{-0.088}^{+0.068}$ & $-0.28_{-0.04}^{+0.04}$ & $1.085_{-0.040}^{+0.043}$ & $5.65_{-0.27}^{+0.22}$ & 7.00 & 125 \\
\hline KELT-7b & 2.735 & $1.280 \pm 0.180$ & $1.533_{-0.047}^{+0.046}$ & $2048 \pm 27$ & $6789_{-49}^{+50}$ & $0.419_{-0.035}^{+0.027}$ & $0.14_{-0.08}^{+0.07}$ & $1.535_{-0.054}^{+0.066}$ & $1.15_{-0.10}^{+0.06}$ & 2.95 & 134 \\
\hline KELT-8b & 3.244 & $0.867_{-0.061}^{+0.065}$ & $1.860_{-0.160}^{+0.180}$ & $1675_{-55}^{+61}$ & $5754_{-55}^{+54}$ & $0.369_{-0.067}^{+0.073}$ & $0.27 \pm 0.04$ & $1.211_{-0.066}^{+0.0078}$ & $4.24_{-0.25}^{+0.29}$ & 5.55 & 141 \\
\hline TrES-1b & 3.030 & $0.752_{-0.046}^{+0.047}$ & $1.067_{-0.021}^{+0.0222}$ & $1140_{-12}^{+13}$ & $5230 \pm 50$ & $2.400_{-0.120}^{+0.014}$ & $0.02 \pm 0.05$ & $0.878_{-0.040}^{+0.038}$ & $1.50_{-0.74}^{+0.82}$ & 17.70 & 11,22 \\
\hline TrES-2b & 2.471 & $1.200_{-0.053}^{+0.051}$ & $1.224 \pm 0.041$ & $1498 \pm 17$ & $5850_{-38}^{+38}$ & $1.372_{-0.059}^{+0.061}$ & $-0.02_{-0.06}^{+0.06}$ & $0.983_{-0.063}^{+0.059}$ & $2.75_{-0.50}^{+0.38}$ & 9.85 & $1,2,11,18,19$ \\
\hline TrES-4b & 3.554 & $0.925_{-0.082}^{+0.081}$ & $1.783_{-0.086}^{+0.093}$ & $1785 \pm 29$ & $6200 \pm 75$ & $0.314_{-0.032}^{+0.034}$ & $0.14 \pm 0.09$ & $1.404_{-0.134}^{+0.066}$ & $2.58_{-0.18}^{+0.16}$ & 3.80 & 4,25 \\
\hline TrES-5b & 1.482 & $1.778 \pm 0.063$ & $1.209 \pm 0.021$ & $1484 \pm 41$ & $5171 \pm 36$ & $1.938_{-0.098}^{+0.104}$ & $0.20 \pm 0.08$ & $0.893 \pm 0.024$ & $5.30_{-0.78}^{+0.84}$ & 18.45 & 83 \\
\hline WASP-100b & 2.849 & $2.030 \pm 0.120$ & $1.690 \pm 0.290$ & $2190 \pm 140$ & $6900 \pm 120$ & $0.280_{-0.070}^{+0.140}$ & $-0.03 \pm 0.10$ & $1.570 \pm 0.100$ & $1.36_{-0.13}^{+0.11}$ & 2.35 & 128 \\
\hline WASP-101b & 3.586 & $0.500 \pm 0.040$ & $1.410 \pm 0.050$ & $1560 \pm 35$ & $6380 \pm 120$ & $0.884 \pm 0.061$ & $0.20 \pm 0.12$ & $1.340 \pm 0.070$ & $0.20_{-0.08}^{+0.26}$ & 4.80 & 128 \\
\hline WASP-103b & 0.926 & $1.490 \pm 0.088$ & $1.528_{-0.047}^{+0.073}$ & $2508_{-70}^{+75}$ & $6110 \pm 160$ & $0.584_{-0.055}^{+0.030}$ & $0.06 \pm 0.13$ & $1.220_{-0.036}^{+0.039}$ & $2.73_{-0.56}^{+0.47}$ & 5.25 & 118 \\
\hline WASP-104b & 1.755 & $1.272 \pm 0.047$ & $1.137 \pm 0.037$ & $1516 \pm 39$ & $5450 \pm 130$ & $1.704 \pm 0.099$ & $0.32 \pm 0.09$ & $1.076 \pm 0.049$ & $1.28_{-1.13}^{+0.74}$ & 12.10 & 121 \\
\hline WASP-106b & 9.290 & $1.925 \pm 0.076$ & $1.085_{-0.028}^{+0.046}$ & $1140 \pm 29$ & $6000 \pm 150$ & $0.628_{-0.055}^{+0.014}$ & $-0.09 \pm 0.09$ & $1.192 \pm 0.054$ & $3.80_{-0.52}^{+0.61}$ & 6.30 & 121 \\
\hline WASP-10b & 3.093 & $3.150_{-0.110}^{+0.130}$ & $1.080 \pm 0.020$ & $1119_{-28}^{+26}$ & $4675 \pm 100$ & $3.099 \pm 0.088$ & $0.03 \pm 0.20$ & $0.750_{-0.028}^{+0.040}$ & $4.79_{-3.04}^{+3.96}$ & 19.95 & $26,36,57$ \\
\hline WASP-117b & 10.022 & $0.276 \pm 0.009$ & $1.021_{-0.065}^{+0.076}$ & $1024_{-26}^{+30}$ & $6040 \pm 90$ & $0.990 \pm 0.140$ & $-0.11 \pm 0.14$ & $1.126 \pm 0.029$ & $2.95_{-0.71}^{+0.051}$ & 7.40 & 120 \\
\hline WASP-119b & 2.500 & $1.230 \pm 0.080$ & $1.400 \pm 0.200$ & $1600 \pm 80$ & $5650 \pm 100$ & $0.760 \pm 0.250$ & $0.14 \pm 0.10$ & $1.020 \pm 0.060$ & $5.60_{-0.75}^{+1.55}$ & 8.90 & 155 \\
\hline WASP-120b & 3.611 & $5.010 \pm 0.260$ & $1.515 \pm 0.083$ & $1890 \pm 50$ & $6450 \pm 120$ & $0.285 \pm 0.031$ & $-0.05 \pm 0.07$ & $1.450 \pm 0.110$ & $2.16 \pm 0.18$ & 3.20 & 147 \\
\hline WASP-121b & 1.275 & $1.183_{-0.062}^{+0.064}$ & $1.865 \pm 0.044$ & $2358 \pm 52$ & $6459 \pm 140$ & $0.617_{-0.013}^{+0.011}$ & $0.13 \pm 0.09$ & $1.353_{-0.079}^{+0.080}$ & $1.18_{-0.36}^{+0.28}$ & 4.05 & 146 \\
\hline WASP-122b & 1.710 & $1.284 \pm 0.051$ & $1.731_{-0.062}^{+0.063}$ & $1960 \pm 50$ & $5774_{-74}^{+75}$ & $0.517_{-0.025}^{+0.025}$ & $0.32_{-0.06}^{+0.06}$ & $1.223_{-0.043}^{+0.038}$ & $4.00_{-0.29}^{+0.31}$ & 6.35 & 147,149 \\
\hline WASP-123b & 2.978 & $0.920 \pm 0.050$ & $1.327 \pm 0.074$ & $1510 \pm 40$ & $5740 \pm 130$ & $0.782 \pm 0.080$ & $0.18 \pm 0.08$ & $1.207 \pm 0.089$ & $4.46_{-0.74}^{+0.58}$ & 8.20 & 147 \\
\hline WASP-124b & 3.373 & $0.600 \pm 0.070$ & $1.240 \pm 0.030$ & $1400 \pm 30$ & $6050 \pm 100$ & $1.397 \pm 0.071$ & $-0.02 \pm 0.11$ & $1.070 \pm 0.050$ & $0.18_{-0.04}^{+0.47}$ & 7.45 & 155 \\
\hline
\end{tabular}


Table 7

(Continued)

\begin{tabular}{|c|c|c|c|c|c|c|c|c|c|c|c|}
\hline Planet & $\begin{array}{r}\text { Period } \\
\text { (days) }\end{array}$ & $\begin{array}{c}M_{p} \\
\left(M_{\mathrm{J}}\right) \\
\end{array}$ & $\begin{array}{c}R_{p} \\
\left(R_{\mathrm{J}}\right) \\
\end{array}$ & $\begin{array}{l}T_{\mathrm{eq}} \\
(\mathrm{K})\end{array}$ & $\begin{array}{c}T_{\text {eff } \star} \\
(\mathrm{K}) \\
\end{array}$ & $\begin{array}{l}\rho_{\star} \\
\left(\mathrm{g} \mathrm{cm}^{-3}\right)\end{array}$ & {$[\mathrm{Fe} / \mathrm{H}]$} & $\begin{array}{c}M_{\star} \\
\left(M_{\odot}\right) \\
\end{array}$ & $\begin{array}{l}\text { Age } \\
(\mathrm{Gyr})\end{array}$ & $\begin{array}{c}t_{\text {tot }} \\
(\mathrm{Gyr})\end{array}$ & References \\
\hline WASP-126b & 3.289 & $0.280 \pm 0.040$ & $0.960_{-0.050}^{+0.100}$ & $1480 \pm 60$ & $5800 \pm 100$ & $0.790_{-0.170}^{+0.080}$ & $0.17 \pm 0.08$ & $1.120 \pm 0.060$ & $4.20_{-0.63}^{+0.41}$ & 8.00 & 155 \\
\hline WASP-12b & 1.091 & $1.410 \pm 0.100$ & $1.790 \pm 0.090$ & $2516 \pm 36$ & $6300_{-100}^{+200}$ & $0.494 \pm 0.042$ & $0.30_{-0.15}^{+0.05}$ & $1.350 \pm 0.140$ & $1.61_{-0.40}^{+0.31}$ & 4.05 & 27 \\
\hline WASP-130b & 11.551 & $1.230 \pm 0.040$ & $0.890 \pm 0.030$ & $833 \pm 18$ & $5600 \pm 100$ & $1.670 \pm 0.130$ & $0.26 \pm 0.10$ & $1.040 \pm 0.040$ & $0.18_{-0.03}^{+0.02}$ & 10.60 & 158 \\
\hline WASP-131b & 5.322 & $0.270 \pm 0.020$ & $1.220 \pm 0.050$ & $1460 \pm 30$ & $5950 \pm 100$ & $0.412 \pm 0.037$ & $-0.18 \pm 0.08$ & $1.060 \pm 0.060$ & $5.80_{-0.62}^{+0.47}$ & 7.20 & 158 \\
\hline WASP-132b & 7.134 & $0.410 \pm 0.030$ & $0.870 \pm 0.030$ & $763 \pm 16$ & $4750 \pm 100$ & $2.823_{-0.200}^{+0.099}$ & $0.22 \pm 0.13$ & $0.800 \pm 0.040$ & $3.17_{-1.92}^{+1.57}$ & 19.95 & 158 \\
\hline WASP-133b & 2.176 & $1.160 \pm 0.090$ & $1.210 \pm 0.050$ & $1790 \pm 40$ & $5700 \pm 100$ & $0.550 \pm 0.040$ & $0.29 \pm 0.12$ & $1.160 \pm 0.080$ & $4.36_{-0.54}^{+0.47}$ & 6.75 & 155 \\
\hline WASP-135b & 1.401 & $1.900 \pm 0.080$ & $1.300 \pm 0.090$ & $1717_{-40}^{+46}$ & $5680 \pm 60$ & $1.580 \pm 0.210$ & $0.02 \pm 0.13$ & $1.010 \pm 0.070$ & $2.16_{-1.11}^{+0.91}$ & 10.70 & 160 \\
\hline WASP-13b & 4.353 & $0.460_{-0.050}^{+0.060}$ & $1.210_{-0.120}^{+0.140}$ & $1417_{-58}^{+62}$ & $5826 \pm 100$ & $0.610_{-0.140}^{+0.170}$ & $0.00 \pm 0.20$ & $1.030_{-0.090}^{+0.110}$ & $4.49_{-0.70}^{+1.97}$ & 6.65 & 17,100 \\
\hline WASP-140b & 2.236 & $2.440 \pm 0.070$ & $1.440_{-0.180}^{+0.420}$ & $1320 \pm 40$ & $5300 \pm 100$ & $1.950 \pm 0.250$ & $0.12 \pm 0.10$ & $0.900 \pm 0.040$ & $2.69_{-1.22}^{+1.17}$ & 15.10 & 158 \\
\hline WASP-141b & 3.311 & $2.690 \pm 0.150$ & $1.210 \pm 0.080$ & $1540 \pm 50$ & $6050 \pm 120$ & $0.692 \pm 0.099$ & $0.29 \pm 0.09$ & $1.250 \pm 0.060$ & $2.15_{-0.50}^{+0.36}$ & 5.65 & 158 \\
\hline WASP-142b & 2.053 & $0.840 \pm 0.090$ & $1.530 \pm 0.080$ & $2000 \pm 60$ & $6060 \pm 150$ & $0.423 \pm 0.056$ & $0.26 \pm 0.12$ & $1.330 \pm 0.080$ & $2.54_{-0.43}^{+0.35}$ & 4.50 & 158 \\
\hline WASP-14b & 2.244 & $7.341_{-0.496}^{+0.508}$ & $1.281_{-0.082}^{+0.075}$ & $1866_{-42}^{+37}$ & $6475 \pm 100$ & $0.765_{-0.085}^{+0.111}$ & $0.00 \pm 0.20$ & $1.211_{-0.122}^{+0.127}$ & $0.79_{-0.40}^{+0.37}$ & 4.30 & 35,39 \\
\hline WASP-157b & 3.952 & $0.574 \pm 0.093$ & $1.045 \pm 0.044$ & $1339 \pm 93$ & $5840 \pm 140$ & $1.300 \pm 0.320$ & $0.34 \pm 0.09$ & $1.260 \pm 0.120$ & $0.18_{-0.04}^{+0.67}$ & 7.65 & 157 \\
\hline WASP-15b & 3.752 & $0.542 \pm 0.050$ & $1.428 \pm 0.077$ & $1652 \pm 28$ & $6300 \pm 100$ & $0.515 \pm 0.052$ & $-0.17 \pm 0.11$ & $1.180 \pm 0.120$ & $2.93_{-0.32}^{+0.25}$ & 4.80 & 21 \\
\hline WASP-16b & 3.119 & $0.855_{-0.076}^{+0.043}$ & $1.008_{-0.060}^{+0.083}$ & $1280_{-21}^{+35}$ & $5700 \pm 150$ & $1.710_{-0.250}^{+0.180}$ & $0.01 \pm 0.10$ & $1.022_{-0.129}^{+0.074}$ & $0.20_{-0.04}^{+1.45}$ & 10.35 & 30 \\
\hline WASP-17b & 3.735 & $0.486 \pm 0.032$ & $1.991 \pm 0.081$ & $1771 \pm 35$ & $6650 \pm 80$ & $0.474 \pm 0.042$ & $-0.19 \pm 0.09$ & $1.306 \pm 0.026$ & $1.99_{-0.19}^{+0.17}$ & 3.75 & 87 \\
\hline WASP-18b & 0.941 & $10.430 \pm 0.380$ & $1.165 \pm 0.057$ & $2384_{-30}^{+58}$ & $6400 \pm 100$ & $0.972 \pm 0.088$ & $0.00 \pm 0.09$ & $1.281 \pm 0.069$ & $0.45_{-0.33}^{+0.20}$ & 5.15 & 33,38 \\
\hline WASP-19b & 0.789 & $1.168 \pm 0.023$ & $1.386 \pm 0.032$ & $2050 \pm 40$ & $5500 \pm 100$ & $1.401_{-0.059}^{+0.066}$ & $0.02 \pm 0.09$ & $0.970 \pm 0.020$ & $6.25_{-1.39}^{+1.34}$ & 13.65 & 44,78 \\
\hline WASP-1b & 2.520 & $0.918_{-0.090}^{+0.091}$ & $1.514_{-0.047}^{+0.052}$ & $1811_{-27}^{+34}$ & $6110 \pm 45$ & $0.549_{-0.059}^{+0.009}$ & $0.23 \pm 0.08$ & $1.301_{-0.047}^{+0.049}$ & $2.69_{-0.23}^{+0.16}$ & 5.30 & $8,11,82$ \\
\hline WASP-20b & 4.900 & $0.313 \pm 0.018$ & $1.458 \pm 0.057$ & $1379 \pm 32$ & $5950 \pm 100$ & $0.631 \pm 0.047$ & $-0.01 \pm 0.06$ & $1.202 \pm 0.040$ & $4.15_{-0.45}^{+0.55}$ & 6.75 & 130 \\
\hline WASP-21b & 4.323 & $0.270 \pm 0.010$ & $1.143_{-0.030}^{+0.045}$ & $1321_{-26}^{+30}$ & $5800 \pm 100$ & $0.920_{-0.085}^{+0.058}$ & $-0.40 \pm 0.10$ & $0.860 \pm 0.040$ & $9.91_{-1.58}^{+1.38}$ & 13.00 & 41,88 \\
\hline WASP-22b & 3.533 & $0.588 \pm 0.017$ & $1.158_{-0.038}^{+0.061}$ & $1466 \pm 34$ & $6000 \pm 100$ & $0.864_{-0.095}^{+0.066}$ & $-0.05 \pm 0.08$ & $1.109 \pm 0.026$ & $3.58_{-0.60}^{+0.42}$ & 7.45 & 43,69 \\
\hline WASP-23b & 2.944 & $0.884_{-0.099}^{+0.088}$ & $0.962_{-0.056}^{+0.047}$ & $1119_{-21}^{+22}$ & $5150 \pm 100$ & $2.601_{-0.038}^{+0.035}$ & $-0.05 \pm 0.13$ & $0.780_{-0.120}^{+0.130}$ & $1.30_{-0.78}^{+1.36}$ & 18.85 & 65 \\
\hline WASP-24b & 2.341 & $1.032_{-0.037}^{+0.038}$ & $1.104_{-0.057}^{+0.052}$ & $1660_{-42}^{+44}$ & $6075 \pm 100$ & $1.054_{-0.100}^{+0.140}$ & $0.07 \pm 0.10$ & $1.129_{-0.025}^{+0.027}$ & $1.27_{-0.46}^{+0.50}$ & 6.55 & 53 \\
\hline WASP-25b & 3.765 & $0.580 \pm 0.040$ & $1.220_{-0.050}^{+0.060}$ & $1212 \pm 35$ & $5703 \pm 100$ & $1.820 \pm 0.140$ & $-0.07 \pm 0.10$ & $1.000 \pm 0.030$ & $1.00_{-0.85}^{+0.65}$ & 11.00 & 86 \\
\hline WASP-26b & 2.757 & $1.028 \pm 0.021$ & $1.281 \pm 0.075$ & $1637 \pm 45$ & $5950 \pm 100$ & $0.709 \pm 0.088$ & $-0.02 \pm 0.09$ & $1.111 \pm 0.028$ & $4.02_{-0.42}^{+0.63}$ & 7.00 & 42,69 \\
\hline WASP-28b & 3.409 & $0.907 \pm 0.043$ & $1.213 \pm 0.042$ & $1468 \pm 37$ & $6150 \pm 140$ & $1.107 \pm 0.082$ & $-0.29 \pm 0.10$ & $1.021 \pm 0.050$ & $2.44_{-0.81}^{+0.68}$ & 7.20 & 130 \\
\hline WASP-29b & 3.923 & $0.244 \pm 0.020$ & $0.792_{-0.035}^{+0.056}$ & $980 \pm 40$ & $4800 \pm 150$ & $2.200_{-0.320}^{+0.280}$ & $0.11 \pm 0.14$ & $0.825 \pm 0.033$ & $11.68_{-4.10}^{+3.31}$ & 19.95 & 54 \\
\hline WASP- $2 b$ & 2.152 & $0.915_{-0.093}^{+0.090}$ & $1.071_{-0.083}^{+0.080}$ & $1304 \pm 54$ & $5200 \pm 200$ & $2.050_{-0.150}^{+0.260}$ & $0.02 \pm 0.05$ & $0.890 \pm 0.120$ & $4.68_{-2.38}^{+2.22}$ & 17.65 & $7,11,82,110$ \\
\hline WASP-30b & 4.157 & $60.960 \pm 0.890$ & $0.889 \pm 0.021$ & $1474 \pm 25$ & $6100 \pm 100$ & $0.758 \pm 0.027$ & $-0.08 \pm 0.10$ & $1.166 \pm 0.026$ & $3.26_{-0.51}^{+0.36}$ & 6.50 & 76 \\
\hline WASP-31b & 3.406 & $0.478 \pm 0.030$ & $1.537 \pm 0.060$ & $1568 \pm 33$ & $6203 \pm 98$ & $0.858 \pm 0.073$ & $-0.19 \pm 0.09$ & $1.161 \pm 0.026$ & $2.86_{-0.53}^{+0.35}$ & 6.55 & 67 \\
\hline WASP-32b & 2.719 & $3.600 \pm 0.070$ & $1.180 \pm 0.070$ & $1560 \pm 50$ & $6100 \pm 100$ & $1.130 \pm 0.140$ & $-0.13 \pm 0.10$ & $1.100 \pm 0.030$ & $1.87_{-0.69}^{+0.61}$ & 7.15 & 62 \\
\hline WASP-34b & 4.318 & $0.590 \pm 0.010$ & $1.220_{-0.080}^{+0.110}$ & $1250 \pm 30$ & $5700 \pm 100$ & $1.770_{-0.563}^{+0.917}$ & $-0.02 \pm 0.10$ & $1.010 \pm 0.070$ & $0.20_{-0.05}^{+1.31}$ & 10.75 & 64 \\
\hline WASP-35b & 3.162 & $0.720 \pm 0.060$ & $1.320 \pm 0.030$ & $1450 \pm 20$ & $6050 \pm 100$ & $1.171 \pm 0.042$ & $-0.15 \pm 0.09$ & $1.070 \pm 0.020$ & $2.31_{-0.72}^{+0.67}$ & 7.75 & 73 \\
\hline WASP-36b & 1.537 & $2.270 \pm 0.068$ & $1.269 \pm 0.030$ & $1700_{-44}^{+42}$ & $5800 \pm 150$ & $1.719_{-0.068}^{+0.075}$ & $-0.31 \pm 0.12$ & $1.020 \pm 0.032$ & $2.56_{-1.30}^{+1.13}$ & 10.60 & 93 \\
\hline WASP-37b & 3.577 & $1.696_{-0.128}^{+0.123}$ & $1.136_{-0.051}^{+0.060}$ & $1325_{-15}^{+25}$ & $5800 \pm 150$ & $0.923_{-0.097}^{+0.064}$ & $-0.40 \pm 0.12$ & $0.849_{-0.040}^{+0.067}$ & $9.06_{-2.09}^{+1.63}$ & 12.10 & 72 \\
\hline WASP-38b & 6.872 & $2.712 \pm 0.065$ & $1.079_{-0.044}^{+0.053}$ & $1261_{-23}^{+24}$ & $6150 \pm 80$ & $0.673 \pm 0.058$ & $-0.12 \pm 0.07$ & $1.216 \pm 0.041$ & $3.42_{-0.30}^{+0.20}$ & 6.10 & 63 \\
\hline WASP-39b & 4.055 & $0.280 \pm 0.030$ & $1.270 \pm 0.040$ & $1116_{-32}^{+33}$ & $5400 \pm 150$ & $1.830_{-0.100}^{+0.120}$ & $-0.12 \pm 0.10$ & $0.930 \pm 0.030$ & $5.31_{-2.17}^{+1.81}$ & 15.40 & 66 \\
\hline WASP-3b & 1.847 & $1.875_{-0.036}^{+0.031}$ & $1.290_{-0.120}^{+0.050}$ & $1960_{-76}^{+33}$ & $6400 \pm 100$ & $0.780_{-0.070}^{+0.210}$ & $0.00 \pm 0.20$ & $1.240_{-0.110}^{+0.060}$ & $0.98_{-0.44}^{+0.46}$ & 4.60 & $9,14,49$ \\
\hline WASP-41b & 3.052 & $0.920 \pm 0.070$ & $1.210 \pm 0.070$ & $1235 \pm 50$ & $5450 \pm 150$ & $1.790 \pm 0.200$ & $-0.08 \pm 0.09$ & $0.940 \pm 0.030$ & $4.36_{-1.78}^{+1.64}$ & 14.30 & 90 \\
\hline WASP-42b & 4.982 & $0.500 \pm 0.035$ & $1.080 \pm 0.057$ & $995 \pm 34$ & $5200 \pm 150$ & $1.930 \pm 0.200$ & $0.05 \pm 0.13$ & $0.881_{-0.081}^{+0.086}$ & $4.96_{-2.27}^{+2.09}$ & 16.90 & 91 \\
\hline WASP-43b & 0.813 & $1.780 \pm 0.100$ & $0.930_{-0.090}^{+0.070}$ & $1370 \pm 70$ & $4400 \pm 200$ & $3.810_{-0.510}^{+0.860}$ & $-0.05 \pm 0.17$ & $0.580 \pm 0.050$ & $6.71_{-5.61}^{+6.35}$ & 19.95 & 70 \\
\hline
\end{tabular}


Table 7

(Continued)

\begin{tabular}{|c|c|c|c|c|c|c|c|c|c|c|c|}
\hline Planet & $\begin{array}{l}\text { Period } \\
\text { (days) }\end{array}$ & $\begin{array}{l}M_{p} \\
\left(M_{\mathrm{J}}\right)\end{array}$ & $\begin{array}{l}R_{p} \\
\left(R_{\mathrm{J}}\right)\end{array}$ & $\begin{array}{l}T_{\mathrm{eq}} \\
(\mathrm{K})\end{array}$ & $\begin{array}{c}T_{\text {eff } \star} \\
(\mathrm{K})\end{array}$ & $\begin{array}{l}\rho_{\star} \\
\left(\mathrm{g} \mathrm{cm}^{-3}\right)\end{array}$ & {$[\mathrm{Fe} / \mathrm{H}]$} & $\begin{array}{c}M_{\star} \\
\left(M_{\odot}\right)\end{array}$ & $\begin{array}{l}\text { Age } \\
(\mathrm{Gyr})\end{array}$ & $\begin{array}{l}t_{\mathrm{tot}} \\
(\mathrm{Gyr})\end{array}$ & References \\
\hline WASP-44b & 2.424 & $0.893_{-0.066}^{+0.071}$ & $1.090_{-0.140}^{+0.130}$ & $1343 \pm 64$ & $5400 \pm 150$ & $1.877_{-0.423}^{+0.762}$ & $0.06 \pm 0.10$ & $0.948 \pm 0.034$ & $2.31_{-2.11}^{+1.24}$ & 13.65 & 101 \\
\hline WASP-45b & 3.126 & $1.005 \pm 0.053$ & $1.170_{-0.140}^{+0.280}$ & $1198 \pm 69$ & $5100 \pm 200$ & $1.496 \pm 0.381$ & $0.36 \pm 0.12$ & $0.910_{-60.000}^{+0.060}$ & $8.29_{-3.22}^{+2.15}$ & 18.05 & 101 \\
\hline WASP-46b & 1.430 & $2.100 \pm 0.073$ & $1.327 \pm 0.058$ & $1654 \pm 50$ & $5600 \pm 150$ & $1.694 \pm 0.169$ & $-0.37 \pm 0.13$ & $0.957 \pm 0.034$ & $6.97_{-2.37}^{+1.90}$ & 14.45 & 101 \\
\hline WASP-47b & 4.159 & $1.164 \pm 0.091$ & $1.134 \pm 0.039$ & $1220 \pm 20$ & $5400 \pm 100$ & $1.017 \pm 0.013$ & $0.18 \pm 0.07$ & $1.084 \pm 0.037$ & $9.83_{-2.04}^{+1.10}$ & 14.95 & $102,142,144$ \\
\hline WASP-47d & 9.031 & $0.027_{-0.011}^{+0.012}$ & $0.321 \pm 0.012$ & $943_{-17}^{+17}$ & $5400 \pm 100$ & $1.017 \pm 0.013$ & $0.18 \pm 0.07$ & $1.084 \pm 0.037$ & $9.79_{-2.01}^{+1.12}$ & 14.95 & 102,142 \\
\hline WASP-47e & 0.790 & $0.038 \pm 0.012$ & $0.162 \pm 0.006$ & $2126_{-39}^{+39}$ & $5400 \pm 100$ & $1.017 \pm 0.013$ & $0.18 \pm 0.07$ & $1.084 \pm 0.037$ & $9.86_{-1.86}^{+1.06}$ & 15.00 & $102,142,144$ \\
\hline WASP-48b & 2.144 & $0.980 \pm 0.090$ & $1.670 \pm 0.080$ & $2030 \pm 70$ & $6000 \pm 150$ & $0.310 \pm 0.028$ & $-0.12 \pm 0.12$ & $1.190 \pm 0.040$ & $4.30_{-1.18}^{+0.78}$ & 5.50 & 73 \\
\hline WASP-49b & 2.782 & $0.378 \pm 0.027$ & $1.115 \pm 0.047$ & $1369 \pm 39$ & $5600 \pm 150$ & $1.425 \pm 0.085$ & $-0.23 \pm 0.07$ & $0.938_{-0.076}^{+0.080}$ & $7.84_{-1.97}^{+1.28}$ & 14.55 & 91 \\
\hline WASP-4b & 1.338 & $1.237 \pm 0.064$ & $1.365 \pm 0.021$ & $1650 \pm 30$ & $5500 \pm 100$ & $1.728_{-0.047}^{+0.016}$ & $-0.03 \pm 0.09$ & $0.925 \pm 0.040$ & $3.79_{-1.28}^{+1.25}$ & 13.45 & 15,20 \\
\hline WASP-50b & 1.955 & $1.468_{-0.086}^{+0.091}$ & $1.153 \pm 0.048$ & $1393 \pm 30$ & $5400 \pm 100$ & $2.090_{-0.130}^{+0.0140}$ & $-0.12 \pm 0.08$ & $0.892_{-0.074}^{+0.080}$ & $3.36_{-1.42}^{+1.28}$ & 15.55 & 68 \\
\hline WASP-52b & 1.750 & $0.460 \pm 0.020$ & $1.270 \pm 0.030$ & $1315 \pm 35$ & $5000 \pm 100$ & $2.480 \pm 0.110$ & $0.03 \pm 0.12$ & $0.870 \pm 0.030$ & $4.06_{-2.23}^{+2.18}$ & 19.95 & 105 \\
\hline WASP-54b & 3.694 & $0.626 \pm 0.023$ & $1.650_{-0.180}^{+0.090}$ & $1742_{-69}^{+49}$ & $6100 \pm 100$ & $0.296_{-0.028}^{+0.085}$ & $-0.27 \pm 0.08$ & $1.201_{-0.036}^{+0.034}$ & $4.91_{-0.44}^{+0.36}$ & 5.80 & 106 \\
\hline WASP-55b & 4.466 & $0.570 \pm 0.040$ & $1.300_{-0.030}^{+0.050}$ & $1290 \pm 25$ & $5900 \pm 100$ & $1.200_{-0.099}^{+0.040}$ & $-0.20 \pm 0.08$ & $1.010 \pm 0.040$ & $4.28_{-1.03}^{+1.12}$ & 9.80 & 102 \\
\hline WASP-56b & 4.617 & $0.571_{-0.035}^{+0.034}$ & $1.092_{-0.033}^{+0.035}$ & $1216_{-24}^{+25}$ & $5600 \pm 100$ & $1.040 \pm 0.060$ & $0.12 \pm 0.06$ & $1.017 \pm 0.024$ & $6.01_{-0.98}^{+1.09}$ & 11.30 & 106 \\
\hline WASP-57b & 2.839 & $0.672_{-0.046}^{+0.049}$ & $0.916_{-0.014}^{+0.017}$ & $1251_{-22}^{+21}$ & $5600 \pm 100$ & $2.319_{-0.089}^{+0.062}$ & $-0.25 \pm 0.10$ & $0.954 \pm 0.027$ & $0.69_{-0.51}^{+0.64}$ & 13.60 & 106 \\
\hline WASP-58b & 5.017 & $0.890 \pm 0.070$ & $1.370 \pm 0.200$ & $1270 \pm 80$ & $5800 \pm 150$ & $0.820 \pm 0.270$ & $-0.45 \pm 0.09$ & $0.940 \pm 0.100$ & $10.21_{-2.11}^{+1.94}$ & 12.75 & 105 \\
\hline WASP-59b & 7.920 & $0.863 \pm 0.045$ & $0.775 \pm 0.068$ & $670 \pm 35$ & $4650 \pm 150$ & $4.390 \pm 0.900$ & $-0.15 \pm 0.11$ & $0.719 \pm 0.035$ & $0.14_{-0.03}^{+0.03}$ & 19.95 & 105 \\
\hline WASP-5b & 1.628 & $1.637 \pm 0.082$ & $1.171 \pm 0.057$ & $1706_{-48}^{+52}$ & $5700 \pm 100$ & $1.130 \pm 0.110$ & $0.09 \pm 0.09$ & $1.021 \pm 0.063$ & $4.45_{-0.94}^{+0.075}$ & 10.20 & $15,37,89$ \\
\hline WASP-60b & 4.305 & $0.514 \pm 0.034$ & $0.860 \pm 0.120$ & $1320 \pm 75$ & $5900 \pm 100$ & $1.020 \pm 0.280$ & $-0.04 \pm 0.09$ & $1.078 \pm 0.035$ & $3.72_{-0.68}^{+0.68}$ & 8.50 & 105 \\
\hline WASP-61b & 3.856 & $2.060 \pm 0.170$ & $1.240 \pm 0.030$ & $1565 \pm 35$ & $6250 \pm 150$ & $0.687_{-0.024}^{+0.011}$ & $-0.10 \pm 0.12$ & $1.220 \pm 0.070$ & $2.51_{-0.53}^{+0.38}$ & 5.35 & 102 \\
\hline WASP-62b & 4.412 & $0.570 \pm 0.040$ & $1.390 \pm 0.060$ & $1440 \pm 30$ & $6230 \pm 80$ & $0.833 \pm 0.085$ & $0.04 \pm 0.06$ & $1.250 \pm 0.050$ & $1.88_{-0.36}^{+0.32}$ & 5.85 & 102 \\
\hline WASP-63b & 4.378 & $0.380 \pm 0.030$ & $1.430_{-0.060}^{+0.100}$ & $1540 \pm 40$ & $5550 \pm 100$ & $0.279_{-0.035}^{+0.024}$ & $0.08 \pm 0.07$ & $1.320 \pm 0.050$ & $7.19_{-0.71}^{+0.53}$ & 8.00 & 102 \\
\hline WASP-64b & 1.573 & $1.271 \pm 0.068$ & $1.271 \pm 0.039$ & $1689 \pm 49$ & $5550 \pm 150$ & $1.198_{-0.062}^{+0.075}$ & $-0.08 \pm 0.11$ & $1.004 \pm 0.028$ & $7.70_{-2.08}^{+1.63}$ & 13.30 & 109 \\
\hline WASP-65b & 2.311 & $1.550 \pm 0.160$ & $1.112 \pm 0.059$ & $1480 \pm 10$ & $5600 \pm 100$ & $1.280_{-0.060}^{+0.040}$ & $-0.07 \pm 0.07$ & $0.930 \pm 0.140$ & $7.25_{-1.44}^{+0.94}$ & 13.65 & 113 \\
\hline WASP-66b & 4.086 & $2.320 \pm 0.130$ & $1.390 \pm 0.090$ & $1790 \pm 60$ & $6600 \pm 150$ & $0.342_{-0.040}^{+0.050}$ & $-0.31 \pm 0.10$ & $1.300 \pm 0.070$ & $2.29_{-0.23}^{+0.20}$ & 3.35 & 102 \\
\hline WASP-67b & 4.614 & $0.420 \pm 0.040$ & $1.400_{-0.200}^{+0.300}$ & $1040 \pm 30$ & $5200 \pm 100$ & $1.860 \pm 0.210$ & $-0.07 \pm 0.09$ & $0.870 \pm 0.040$ & $9.66_{-2.28}^{+1.77}$ & 19.95 & 102 \\
\hline WASP-68b & 5.084 & $0.950 \pm 0.030$ & $1.270_{-0.060}^{+0.110}$ & $1488_{-32}^{+49}$ & $5910 \pm 60$ & $0.367_{-0.071}^{+0.042}$ & $0.22 \pm 0.08$ & $1.240 \pm 0.030$ & $3.58_{-0.23}^{+0.13}$ & 4.95 & 119 \\
\hline WASP-69b & 3.868 & $0.260 \pm 0.017$ & $1.057 \pm 0.047$ & $963 \pm 18$ & $4700 \pm 50$ & $2.170 \pm 0.180$ & $0.15 \pm 0.08$ & $0.826 \pm 0.029$ & $18.46_{-2.04}^{+1.212}$ & 19.95 & 129 \\
\hline WASP-6b & 3.361 & $0.503_{-0.038}^{+0.019}$ & $1.224_{-0.052}^{+0.051}$ & $1194_{-57}^{+58}$ & $5450 \pm 100$ & $1.890_{-0.140}^{+0.160}$ & $-0.20 \pm 0.09$ & $0.880_{-0.080}^{+0.050}$ & $5.94_{-1.83}^{+1.37}$ & 16.05 & 16 \\
\hline WASP-70Ab & 3.713 & $0.590 \pm 0.022$ & $1.164_{-0.102}^{+0.073}$ & $1387 \pm 40$ & $5700 \pm 80$ & $0.870_{-0.110}^{+0.190}$ & $-0.01 \pm 0.06$ & $1.106 \pm 0.042$ & $7.57_{-1.10}^{+0.80}$ & 11.40 & 129 \\
\hline WASP-71b & 2.904 & $2.258 \pm 0.072$ & $1.500 \pm 0.110$ & $2066 \pm 67$ & $6050 \pm 100$ & $0.179 \pm 0.030$ & $0.15 \pm 0.07$ & $1.572 \pm 0.062$ & $2.50_{-0.17}^{+0.18}$ & 3.00 & 108 \\
\hline WASP-72b & 2.217 & $1.410_{-0.045}^{+0.050}$ & $1.010_{-0.080}^{+0.120}$ & $2064_{-62}^{+90}$ & $6250 \pm 100$ & $0.374_{-0.080}^{+0.062}$ & $-0.06 \pm 0.09$ & $1.327_{-0.035}^{+0.043}$ & $2.87_{-0.27}^{+0.18}$ & 4.15 & 109 \\
\hline WASP-73b & 4.087 & $1.880_{-0.060}^{+0.070}$ & $1.160_{-0.080}^{+0.120}$ & $1790_{-51}^{+75}$ & $6030 \pm 120$ & $0.212_{-0.056}^{+0.028}$ & $0.14 \pm 0.14$ & $1.340_{-0.040}^{+0.050}$ & $2.62_{-0.29}^{+0.22}$ & 3.25 & 119 \\
\hline WASP-74b & 2.138 & $0.950 \pm 0.060$ & $1.560 \pm 0.060$ & $1910 \pm 40$ & $5990 \pm 110$ & $0.477 \pm 0.025$ & $0.39 \pm 0.13$ & $1.480 \pm 0.120$ & $2.45_{-0.35}^{+0.30}$ & 4.90 & 136 \\
\hline WASP-75b & 2.484 & $1.070 \pm 0.050$ & $1.270 \pm 0.048$ & $1710 \pm 20$ & $6100 \pm 100$ & $0.790 \pm 0.060$ & $0.07 \pm 0.09$ & $1.140 \pm 0.070$ & $2.51_{-0.53}^{+0.38}$ & 6.20 & 113 \\
\hline WASP-76b & 1.810 & $0.920 \pm 0.030$ & $1.830_{-0.040}^{+0.060}$ & $2160 \pm 40$ & $6250 \pm 100$ & $0.404_{-0.025}^{+0.011}$ & $0.23 \pm 0.10$ & $1.460 \pm 0.070$ & $2.15_{-0.25}^{+0.24}$ & 3.95 & 152 \\
\hline WASP-77Ab & 1.360 & $1.760 \pm 0.060$ & $1.210 \pm 0.020$ & $1669_{-24}^{+24}$ & $5500 \pm 80$ & $1.633_{-0.028}^{+0.023}$ & $0.00 \pm 0.11$ & $1.002 \pm 0.045$ & $4.40_{-1.20}^{+1.15}$ & 13.45 & 117 \\
\hline WASP-78b & 2.175 & $0.890 \pm 0.080$ & $1.700 \pm 0.110$ & $2350 \pm 80$ & $6100 \pm 150$ & $0.176 \pm 0.025$ & $-0.35 \pm 0.14$ & $1.330 \pm 0.090$ & $3.82_{-0.38}^{+0.40}$ & 4.10 & 92 \\
\hline WASP-79b & 3.662 & $0.900 \pm 0.080$ & $2.090 \pm 0.140$ & $1900 \pm 50$ & $6600 \pm 100$ & $0.310 \pm 0.040$ & $0.03 \pm 0.10$ & $1.520 \pm 0.070$ & $1.69_{-0.16}^{+0.17}$ & 2.90 & 92 \\
\hline WASP-7b & 4.955 & $0.960_{-0.180}^{+0.120}$ & $0.915_{-0.040}^{+0.046}$ & $1379_{-23}^{+35}$ & $6400 \pm 100$ & $0.929_{-0.179}^{+0.152}$ & $0.00 \pm 0.10$ & $1.280_{-0.190}^{+0.090}$ & $0.57_{-0.37}^{+0.26}$ & 5.00 & 24 \\
\hline WASP-80b & 3.068 & $0.554_{-0.039}^{+0.030}$ & $0.952_{-0.027}^{+0.026}$ & $814_{-19}^{+19}$ & $4145 \pm 100$ & $4.400_{-0.028}^{+0.030}$ & $-0.14 \pm 0.16$ & $0.570 \pm 0.050$ & $15.95_{-6.17}^{+3.43}$ & 19.95 & 107 \\
\hline WASP-82b & 2.706 & $1.240 \pm 0.040$ & $1.670_{-0.050}^{+0.070}$ & $2190 \pm 40$ & $6500 \pm 80$ & $0.223_{-0.020}^{+0.008}$ & $0.12 \pm 0.11$ & $1.630 \pm 0.080$ & $1.70_{-0.14}^{+0.13}$ & 2.55 & 152 \\
\hline
\end{tabular}


Table 7

(Continued)

\begin{tabular}{|c|c|c|c|c|c|c|c|c|c|c|c|}
\hline Planet & $\begin{array}{l}\text { Period } \\
\text { (days) }\end{array}$ & $\begin{array}{c}M_{p} \\
\left(M_{\mathrm{J}}\right)\end{array}$ & $\begin{array}{l}R_{p} \\
\left(R_{\mathrm{J}}\right)\end{array}$ & $\begin{array}{l}T_{\mathrm{eq}} \\
(\mathrm{K})\end{array}$ & $\begin{array}{c}T_{\text {eff } \star} \\
(\mathrm{K})\end{array}$ & $\begin{array}{l}\rho_{\star} \\
\left(\mathrm{g} \mathrm{cm}^{-3}\right)\end{array}$ & {$[\mathrm{Fe} / \mathrm{H}]$} & $\begin{array}{c}M_{\star} \\
\left(M_{\odot}\right)\end{array}$ & $\begin{array}{l}\text { Age } \\
(\mathrm{Gyr})\end{array}$ & $\begin{array}{c}t_{\text {tot }} \\
(\mathrm{Gyr})\end{array}$ & References \\
\hline WASP-83b & 4.971 & $0.300 \pm 0.030$ & $1.040_{-0.050}^{+0.080}$ & $1120 \pm 30$ & $5480 \pm 110$ & $1.369_{-0.180}^{+0.100}$ & $0.29 \pm 0.12$ & $1.110 \pm 0.090$ & $3.95_{-1.13}^{+0.84}$ & 12.20 & 136 \\
\hline WASP-88b & 4.954 & $0.560 \pm 0.080$ & $1.700_{-0.070}^{+0.130}$ & $1772_{-45}^{+54}$ & $6430 \pm 130$ & $0.226_{-0.042}^{+0.028}$ & $-0.08 \pm 0.12$ & $1.450 \pm 0.050$ & $2.07_{-0.19}^{+0.17}$ & 2.80 & 119 \\
\hline WASP-89b & 3.356 & $5.900 \pm 0.400$ & $1.040 \pm 0.040$ & $1120 \pm 20$ & $4955 \pm 100$ & $1.920 \pm 0.099$ & $0.15 \pm 0.14$ & $0.920 \pm 0.080$ & $10.68_{-2.64}^{+2.08}$ & 19.95 & 136 \\
\hline WASP-8b & 8.159 & $2.247_{-0.074}^{+0.083}$ & $1.048_{-0.079}^{+0.042}$ & $951_{-28}^{+21}$ & $5600 \pm 80$ & $1.680_{-0.160}^{+0.310}$ & $0.17 \pm 0.07$ & $1.033_{-0.050}^{+0.058}$ & $1.04_{-0.88}^{+0.64}$ & 11.10 & 40 \\
\hline WASP-90b & 3.916 & $0.630 \pm 0.070$ & $1.630 \pm 0.090$ & $1840 \pm 50$ & $6440 \pm 130$ & $0.282 \pm 0.028$ & $0.11 \pm 0.14$ & $1.550 \pm 0.100$ & $1.79_{-0.26}^{+0.20}$ & 2.90 & 152 \\
\hline WASP-94Ab & 3.950 & $0.452_{-0.032}^{+0.035}$ & $1.720_{-0.050}^{+0.060}$ & $1604_{-22}^{+25}$ & $6153_{-76}^{+75}$ & $0.486_{-0.028}^{+0.016}$ & $0.26 \pm 0.15$ & $1.450 \pm 0.090$ & $2.27_{-0.39}^{+0.28}$ & 4.60 & 122 \\
\hline WASP-95b & 2.185 & $1.130_{-0.040}^{+0.100}$ & $1.210 \pm 0.060$ & $1570 \pm 50$ & $5830 \pm 140$ & $1.100_{-0.180}^{+0.060}$ & $0.14 \pm 0.16$ & $1.110 \pm 0.090$ & $2.35_{-1.12}^{+0.80}$ & 7.90 & 128 \\
\hline WASP-96b & 3.425 & $0.480 \pm 0.030$ & $1.200 \pm 0.060$ & $1285 \pm 40$ & $5500 \pm 150$ & $1.300 \pm 0.100$ & $0.14 \pm 0.19$ & $1.060 \pm 0.090$ & $4.46_{-1.51}^{+1.36}$ & 11.60 & 128 \\
\hline WASP-97b & 2.073 & $1.320 \pm 0.050$ & $1.130 \pm 0.060$ & $1555 \pm 40$ & $5670 \pm 110$ & $1.310 \pm 0.130$ & $0.23 \pm 0.11$ & $1.120 \pm 0.060$ & $2.40_{-0.95}^{+0.77}$ & 9.75 & 128 \\
\hline WASP-98b & 2.963 & $0.830 \pm 0.070$ & $1.100 \pm 0.040$ & $1180 \pm 30$ & $5550 \pm 140$ & $2.810 \pm 0.100$ & $-0.60 \pm 0.19$ & $0.690 \pm 0.060$ & $2.19_{-1.73}^{+2.37}$ & 16.00 & 128 \\
\hline WASP-99b & 5.753 & $2.780 \pm 0.130$ & $1.100_{-0.050}^{+0.080}$ & $1480 \pm 40$ & $6150 \pm 100$ & $0.381_{-0.056}^{+0.028}$ & $0.21 \pm 0.15$ & $1.480 \pm 0.100$ & $2.41_{-0.33}^{+0.31}$ & 4.05 & 128 \\
\hline
\end{tabular}

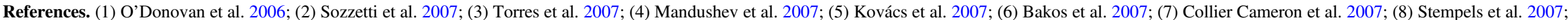

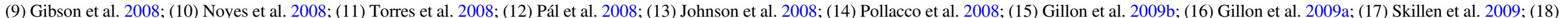

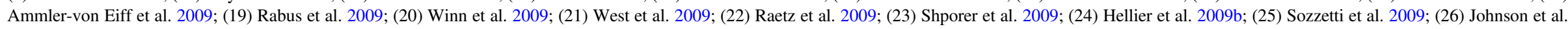

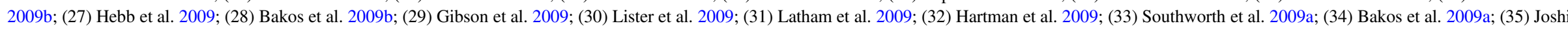

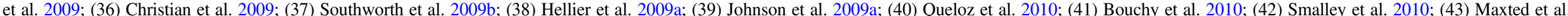

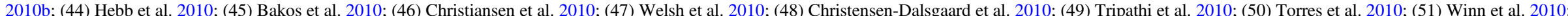

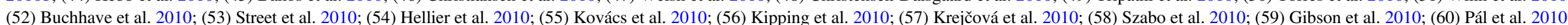

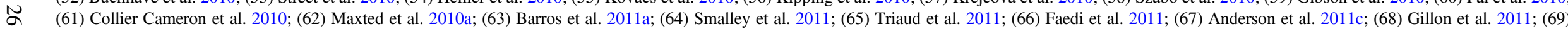

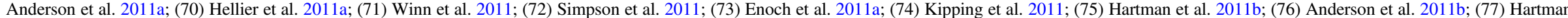

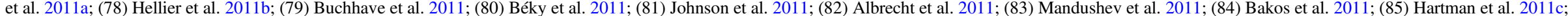

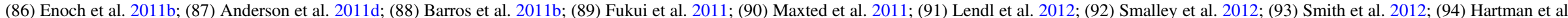

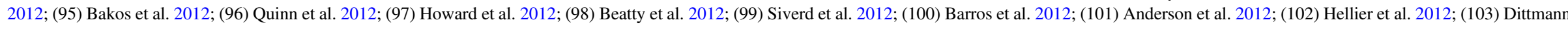

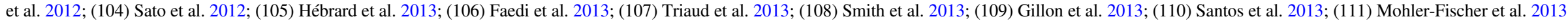

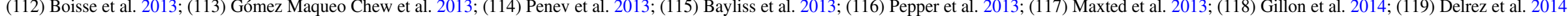

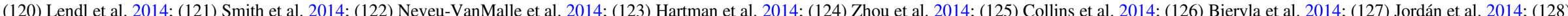

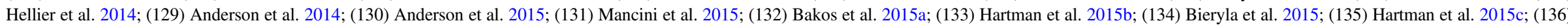

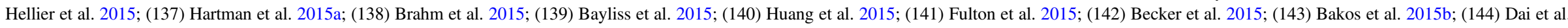

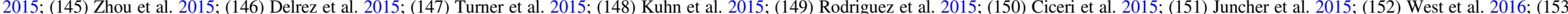

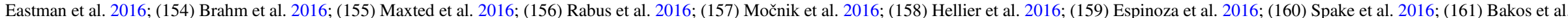
2016; (162) G. Á. Bakos et al. 2016, in preparation); (163) Bento et al. 2016; (164) Bhatti et al. 2016; (165) de Val-Borro et al. 2016; (166) this work; (167) Penev et al. 2016. 
Table 8

Parameter Estimates and Bayesian Evidence for Models of the Form Equation (1)

\begin{tabular}{lccc}
\hline \hline $\begin{array}{l}\ln T_{\text {eq, now }} \\
c_{1}\end{array}$ & $\begin{array}{c}\ln T_{\text {eq,ZAMS }} c_{2} \\
c_{2}\end{array}$ & $\begin{array}{c}\ln a \\
c_{3}\end{array}$ & $\begin{array}{c}\tau \\
c_{4}\end{array}$ \\
\hline $0.92 \pm 0.12$ & 0 & $0.273 \pm 0.075$ & 0 \\
$0.81 \pm 0.14$ & 0 & $0.216 \pm 0.086$ & $0.107 \pm 0.088$ \\
Evidence
\end{tabular}

Note. The models tested are sorted from highest to lowest evidence. The Bayesian evidence is reported relative to that for a model with only a freely varying mean for the $\ln R_{p}$ values. For each parameter we report the mean and standard deviation of its posterior probability distribution.

radius planets, the difference is too small to be responsible for the detected trend between planet radius and fractional host age. Moreover, the difference is also consistent with more evolved/luminous stars generally being more distant from the solar system than less evolved stars, and thus exhibiting greater reddening. No systematic difference is seen in the host star metallicity distributions of small- and large-radius planets (Figure 19).

Priors Used in Stellar Modeling: Stars evolve faster as they age such that a large area on the Hertzsprung-Russell diagram is covered by stellar models spanning a small range of ages near the end of a star's life. As a result, when observed stellar properties are compared to models, there can be a bias toward matching to late ages. This well-known effect, dubbed the stellar terminal age bias by Pont \& Eyer (2004), can be corrected by adopting appropriate priors on the model parameters (e.g., adopting a uniform prior on the age). Similarly, failing to account for the greater prevalence of low-mass stars in the Galaxy relative to high-mass stars can lead to overpredicting stellar masses (e.g., Lloyd 2011). For most transiting planets in the literature, these possible biases have not been accounted for in performing this comparison (i.e., generally the analyses have adopted uniform priors on the relevant observables, namely, the effective temperature, density, and metallicity). While these effects could lead to overestimated stellar radii, potentially explaining the preference for large-radius planets around evolved stars, in practice we only expect such biases to be significant if the observed parameters are not well constrained relative to the scale over which the astrophysically motivated priors change substantially. To estimate the importance of this effect, we calculate new stellar parameters for each of the HAT TEP systems with $R>1.5 R_{\mathrm{J}}$. Here we place a uniform prior on the stellar age between the minimum age of the isochrones and 14 Gyr (this amounts to assuming a constant star formation rate over this period), we use the Chabrier (2003) initial mass function to place a prior on the stellar mass, and we assume a Gaussian prior on the metallicity with a mean of $[\mathrm{Fe} / \mathrm{H}]=0 \operatorname{dex}$ and a standard deviation of 0.5 dex. The details of how we implement this are described in the Appendix.

We find that for all 17 systems the changes to the parameters are much smaller than the uncertainties (the changes are all well below $0.1 \%$, and in most cases below $0.01 \%$ ). We also find that the prior on the stellar mass, which increases toward lowermass stars, generally has a larger impact than the priors on the age or metallicity. The result is that in most cases the stellar masses and radii are very slightly lower when priors are placed on the stellar properties, while the ages are very slightly higher. The latter is due to the prior on stellar mass pulling the solution toward lower effective temperatures, which at the measured stellar densities requires higher ages. We conclude that since the changes in the stellar parameters are insignificant, the correlation between the planetary radii and host star fractional age is not due to biases in the stellar parameters stemming from using incorrect priors.

\subsubsection{Theoretical Significance}

As we have shown, there is a significant correlation between the radii of close-in giant planets and the fractional ages of their host stars. This correlation is apparently a by-product of the more fundamental correlation between planet radius and equilibrium temperature, but the data also indicate that planetary radii increase over time as their host stars evolve and become more luminous.

Such an effect is contrary to models of planet evolution where excess energy associated with a planet's proximity to its host star does not penetrate deep into the planet interior, but only acts to slow the planet's contraction. Burrows et al. (2000) and Baraffe et al. (2008) are examples of such "default" models. Other examples of such models include Burrows et al. (2007), who showed, among other things, how additional opacity, which further slows the contraction, could explain the radii of inflated planets known at that time, and Ibgui et al. (2010), who showed how extended tidal heating of the planet atmosphere can increase the final "equilibrium" radius of a planet. More generally, Spiegel \& Burrows (2013) explored a variety of effects related to planet inflation, including the effect 


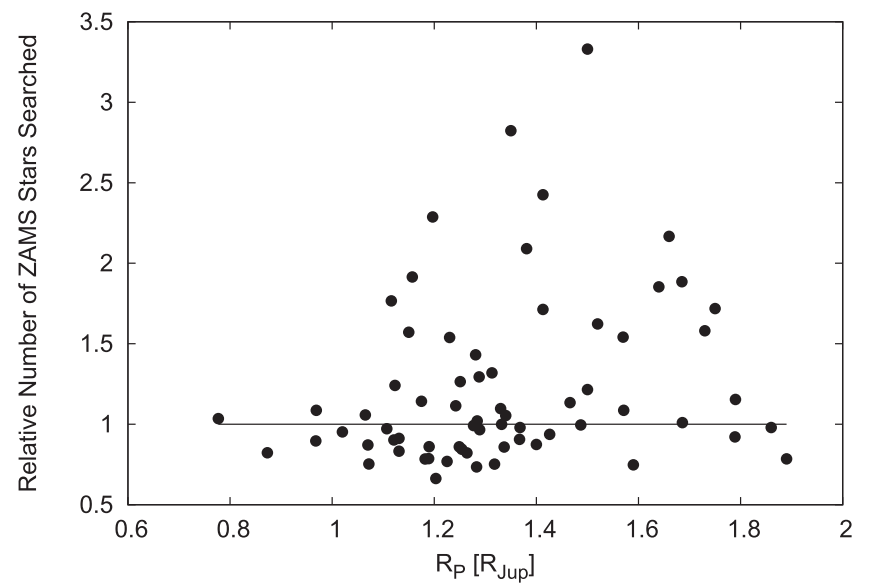

Figure 15. Relative number of ZAMS-equivalent stars searched for a given TEP discovered by HAT to the same transit $\mathrm{S} / \mathrm{N}$ as the observed TEP system (Equation (4)) computed as described in Section 4.1.2. This is shown as a function of planet radius. For the largest planets with $R_{P}>1.5 R_{\mathrm{J}}$ the HAT survey is more sensitive to finding the same planet around a ZAMS star than it is to finding the planet around the moderately evolved star where it was discovered.

on planetary evolution of varying the depth at which additional energy is deposited in the interior (see also Lopez \& Fortney 2016, for a recent discussion). If the inflation mechanism only slows, but does not reverse, the contraction, one would expect that at fixed semimajor axis, older planets should be smaller than younger planets, despite the increase in equilibrium temperature as the host stars evolve. This is not what we see (Figure 11; see also Section 4.1.1).

On the other hand, theories in which the energy is deposited deep in the core of the planet may allow planets to become more inflated as the energy source increases over time (Spiegel \& Burrows 2013). Examples of such models include tidal heating of an eccentric planet's core as considered by Bodenheimer et al. (2001), Liu et al. (2008), and Ibgui et al. (2011), or the ohmic heating model proposed by Batygin \& Stevenson (2010) and Batygin et al. (2011) (though see Huang \& Cumming [2012] and Wu \& Lithwick [2013], who argue that this mechanism cannot heat the deep interior). Our finding that planets apparently reinflate over time is evidence that some mechanism of this type is in operation.

\section{SUMMARY}

The existence of highly inflated close-in giant planets is one of the long-standing mysteries that has emerged in the field of exoplanets. By continuing to build up the sample of inflated planets, we are beginning to see patterns in their properties, allowing us to narrow down the physical processes responsible for the inflation. Here we presented the discovery of two transiting highly inflated planets, HAT-P-65b and HAT-P-66b. The planets are both around moderately evolved stars, which we find to be a general trend-highly inflated planets with $R \gtrsim 1.5 R_{\mathrm{J}}$ have been preferentially found around moderately evolved stars compared to smaller-radius planets. This effect is independently seen in the samples of planets found by HAT, WASP, Kepler, TrES, and KELT. We argue that this is not due to observational selection effects, which tend to favor the discovery of large planets around younger stars, nor is it likely to be the result of systematic errors in the planetary or stellar parameters. We find that the correlation can be explained as a by-product of the more fundamental, and well-known, correlation between planet radius and equilibrium temperature, and that the present-day equilibrium temperature of close-in giant planets, which increases with time as host stars evolve, provides a significantly better predictor of planet radii than does the initial equilibrium temperature at the ZAMS.

We conclude that, after contracting during the pre-mainsequence, close-in giant planets are reinflated over time as their host stars evolve. This provides evidence that the mechanism responsible for this inflation deposits energy deep within the planetary interiors.

The result presented in this paper motivates further observational work to discover and characterize highly inflated planets. In particular, more work is needed to determine the timescale for planet reinflation. The expected release of accurate parallaxes for these systems from Gaia should enable more precise ages for all of these systems. Many more systems are needed to trace the evolution of planet radius with age as a function of planetary mass, host star mass, orbital separation, and other potentially important parameters. Furthermore, the evidence for planetary reinflation presented here provides additional motivation to search for highly inflated long-period planets transiting giant stars.

HATNet operations have been funded by NASA grants NNG04GN74G and NNX13AJ15G. Follow-up of HATNet targets has been partially supported through NSF grant AST1108686. G.Á.B., Z.C., and K.P. acknowledge partial support from NASA grant NNX09AB29G. J.H. acknowledges support from NASA grant NNX14AE87G. K.P. acknowledges support from NASA grant NNX13AQ62G. We acknowledge partial support also from the Kepler Mission under NASA Cooperative Agreement NCC2-1390 (DWL, PI). A.S. is supported by the European Union under a Marie Curie Intra-European Fellowship for Career Development with reference FP7PEOPLE-2013-IEF, number 627202. Part of this work was supported by Fundação para a Ciência e a Tecnologia (FCT, Portugal, ref. UID/FIS/04434/2013) through national funds and by FEDER through COMPETE2020 (ref. POCI-01-0145FEDER-007672). Data presented in this paper are based on observations obtained at the HAT station at the Submillimeter Array of SAO and the HAT station at the Fred Lawrence Whipple Observatory of SAO. This research has made use of the NASA Exoplanet Archive, which is operated by the California Institute of Technology, under contract with the National Aeronautics and Space Administration under the Exoplanet Exploration Program. Data presented herein were obtained at the WIYN Observatory from telescope time allocated to NN-EXPLORE through the scientific partnership of the National Aeronautics and Space Administration, the National Science Foundation, and the National Optical Astronomy Observatory. This work was supported by a NASA WIYN PI Data Award, administered by the NASA Exoplanet Science Institute. We gratefully acknowledge R. W. Noyes for his many contributions to the HATNet transit survey, and we also gratefully acknowledge contributions from J. Johnson and from G. Marcy to the collection and reduction of the Keck/ HIRES observations presented here. The authors wish to recognize and acknowledge the very significant cultural role and reverence that the summit of Mauna Kea has always had within the indigenous Hawaiian community. We are most 
All HAT Planets

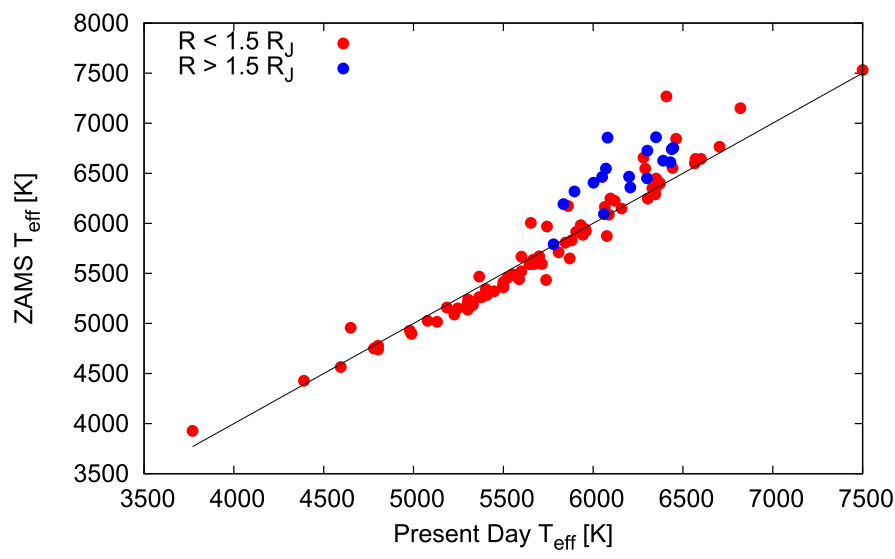

HAT Planets with $\mathrm{T}_{\text {eff,ZAMs }}>6250 \mathrm{~K}$

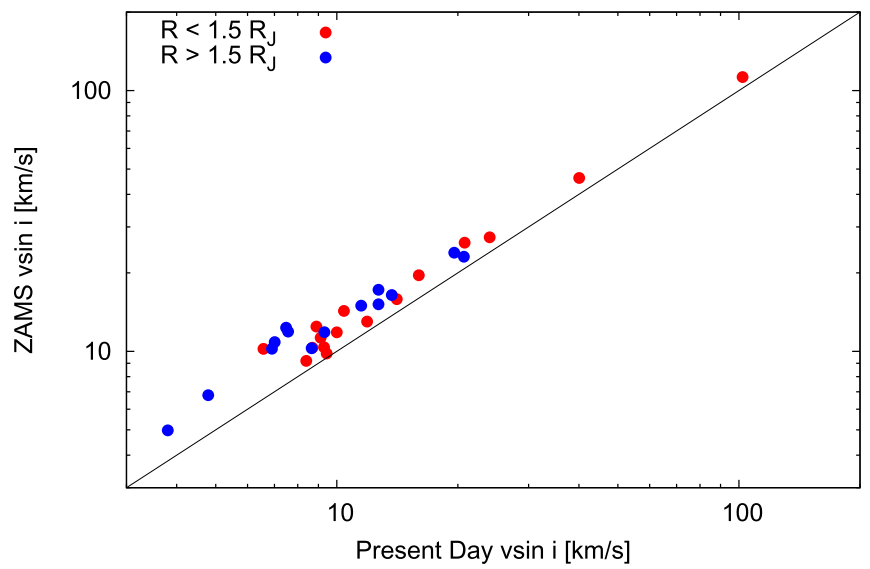

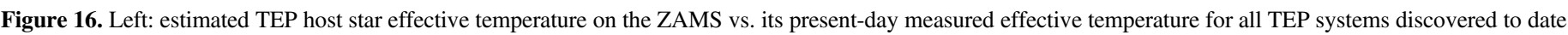

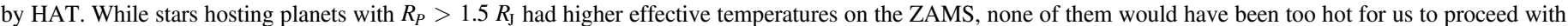

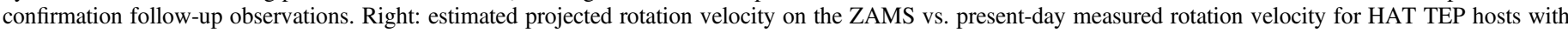

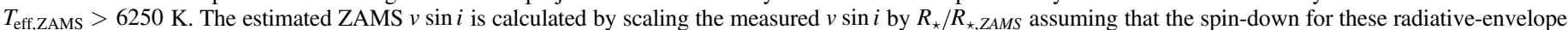

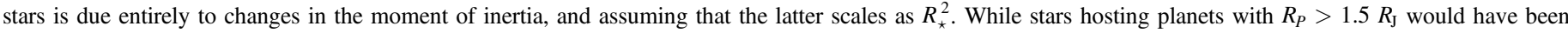

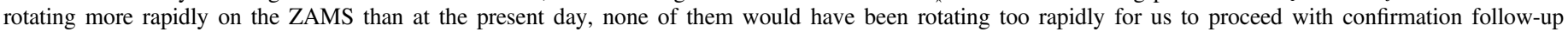
observations.

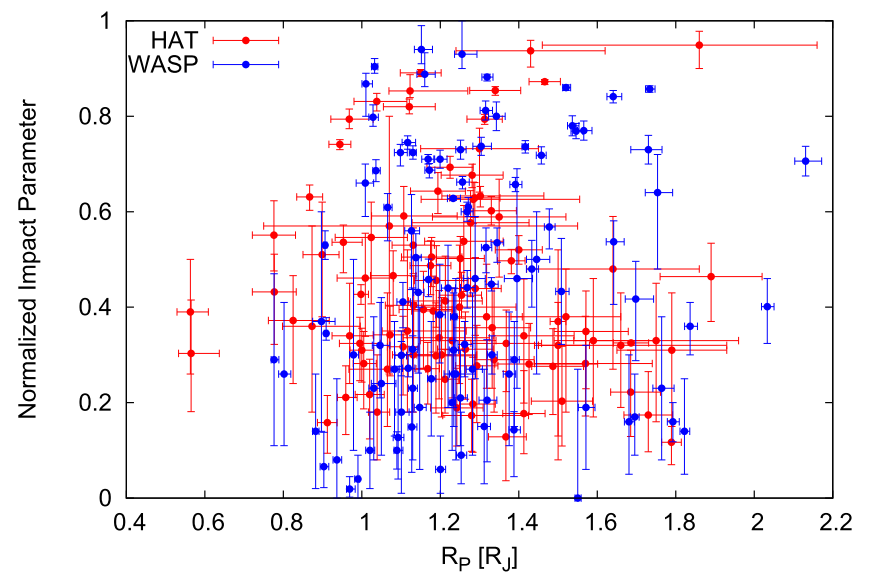

Figure 17. Normalized impact parameter vs. planetary radius for TEPs found by HAT and WASP. The impact parameters of WASP TEPs appear to be uniformly distributed between 0 and 1 , as expected for random orientations in space. The largest HAT planets have, if anything, a bias toward low impact parameters. If these suffer from a systematic error, the stellar and planetary radii would be underestimated.

fortunate to have the opportunity to conduct observations from this mountain.

\section{APPENDIX \\ ESTIMATING TRANSITING PLANET HOST STAR PARAMETERS WITH PRIORS ON THE STELLAR MASS, AGE, AND METALLICITY}

The physical parameters of transiting planet host stars are determined by comparing the observed parameters $T_{\text {eff } \star}, \rho_{\star}$, and $[\mathrm{Fe} / \mathrm{H}]$ to theoretical stellar evolution models. In practice, the light-curve analysis produces a Markov Chain of $\rho_{\star}$ values, which we combine with simulated chains of $T_{\text {eff } \star}$ and $[\mathrm{Fe} / \mathrm{H}]$ values (we assume that the three parameters are uncorrelated, and that $T_{\mathrm{eff} \star}$ and $[\mathrm{Fe} / \mathrm{H}]$ have Gaussian uncertainties). For a given $\left(\rho_{\star}, T_{\text {eff } \star},[\mathrm{Fe} / \mathrm{H}]\right)$ link in the chain, we perform a trilinear interpolation within a grid of isochrones from the YY models to get the corresponding stellar mass, age, radius, luminosity,

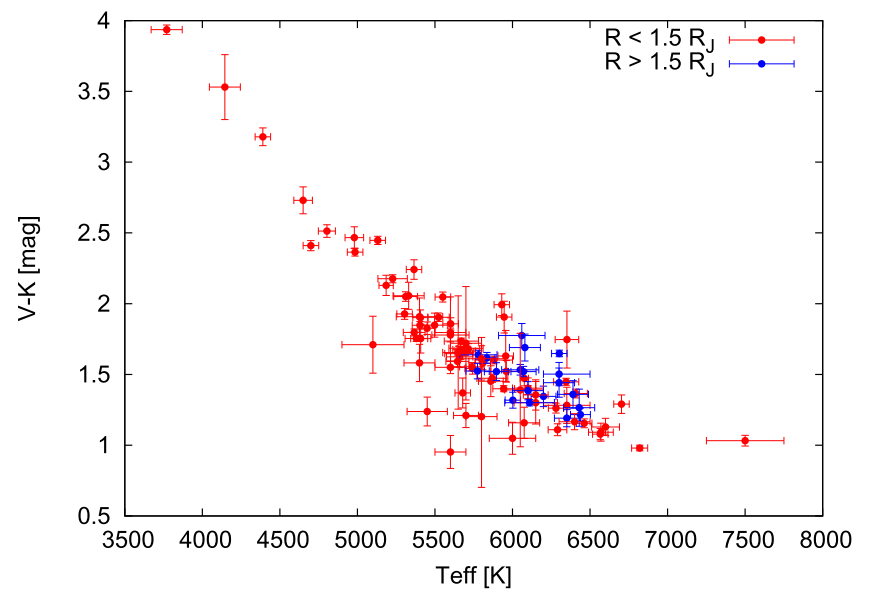

Figure 18. Photometric $V-K$ color (not corrected for reddening) vs. effective temperature for TEP host stars from HAT and WASP with $R_{P}>0.5 R_{\mathrm{J}}$ and $P<10$ days. The blue and red colors are used to distinguish between stars hosting planets with $R_{P}>1.5 R_{\mathrm{J}}$ and $R_{P}<1.5 R_{\mathrm{J}}$, respectively. No systematic difference in the color-temperature relation is seen between these two classes of planets. Such a difference might have indicated a systematic error in the stellar effective temperature measurements of the stars hosting large planets.

and absolute magnitudes in various passbands. Our interpolation routine can use any combination of three parameters as the independent variables; below we make use of this feature using the mass, age, and metallicity as the independent variables. The resulting chain of stellar physical parameters is then used to provide best estimates and uncertainties for each of these parameters.

As discussed in Section 4.1.3, this process may lead to systematic errors in the stellar parameters if priors are not adopted to account for the intrinsic distribution of stars in the Galaxy. The prior is applied as a multiplicative weight that is associated with each link in the Markov Chain. The weights are calculated as follows.

Let $P_{m}(m), P_{t}(t)$, and $P_{[\mathrm{Fe} / \mathrm{H}]}([\mathrm{Fe} / \mathrm{H}])$ be prior probability densities to be placed on the stellar mass, age, and metallicity, respectively. Here we use the Chabrier (2003) initial mass 


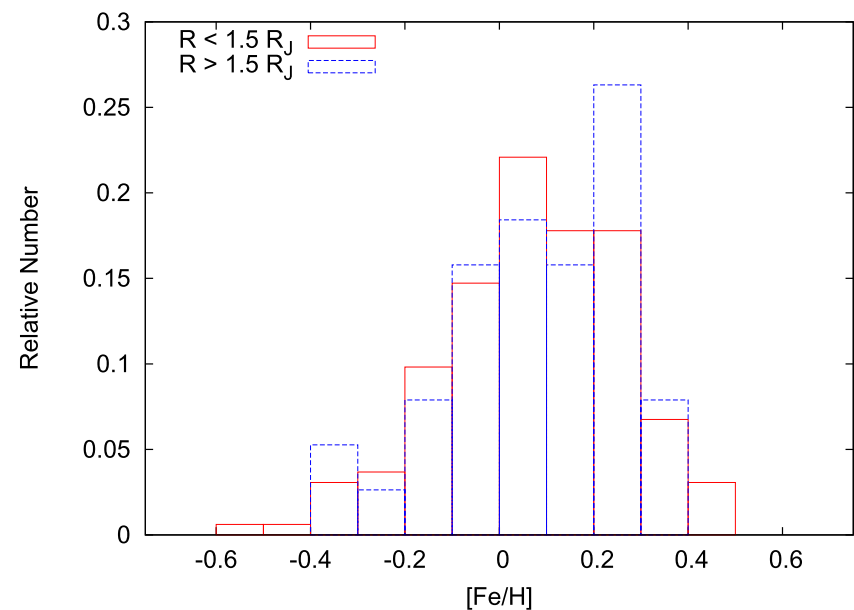

Figure 19. Normalized histograms of $[\mathrm{Fe} / \mathrm{H}]$ for transiting planet host stars from HAT and WASP, separated by planetary radius. No significant difference is seen between metallicities of large-planet-radius host stars and small-planetradius host stars.

function for the prior on the stellar mass, a uniform distribution for the prior on the stellar age, and a Gaussian distribution with mean 0 dex and standard deviation 0.5 dex for the prior on the metallicity. Further, let $C_{m}(m), C_{t}(t)$, and $C_{[\mathrm{Fe} / \mathrm{H}]}([\mathrm{Fe} / \mathrm{H}])$ be the corresponding cumulative distributions of these prior probability densities.

For a given $(m, t,[\mathrm{Fe} / \mathrm{H}])$ link generated from an input set of $\left(\rho_{\star}, T_{\text {eff } \star},[\mathrm{Fe} / \mathrm{H}]\right)$, we find $m^{+}=C_{m}^{-1}\left(C_{m}(m)+\Delta u_{m}\right), t^{+}=$ $C_{t}^{-1}\left(C_{t}(t)+\Delta u_{t}\right)$, and $[\mathrm{Fe} / \mathrm{H}]^{+}=C_{[\mathrm{Fe} / \mathrm{H}]}^{-1}\left(C_{[\mathrm{Fe} / \mathrm{H}]}([\mathrm{Fe} / \mathrm{H}])+\right.$ $\left.\Delta u_{[\mathrm{Fe} / \mathrm{H}]}\right)$ for some small probability steps $\Delta u_{m} \ll 1, \Delta u_{t} \ll 1$, and $\Delta u_{[\mathrm{Fe} / \mathrm{H}]} \ll 1$. Likewise, we calculate $m^{-}, t^{-}$, and $[\mathrm{Fe} / \mathrm{H}]^{-}$ for a negative $\Delta u$. We then perform trilinear interpolation within the isochrones to find $\left(\rho_{m+}, T_{\mathrm{eff}, m+},[\mathrm{Fe} / \mathrm{H}]_{m+}\right)$ associated with the point $\left(m^{+}, t,[\mathrm{Fe} / \mathrm{H}]\right)$, and similarly for $m^{-}, t^{+}$, etc.

Letting

$$
\begin{gathered}
\boldsymbol{v}_{m}=\left(\rho_{m+}-\rho_{m-}, T_{\mathrm{eff}, m+}-T_{\mathrm{eff}, m-},\right. \\
\left.[\mathrm{Fe} / \mathrm{H}]_{m+}-[\mathrm{Fe} / \mathrm{H}]_{m-}\right)
\end{gathered}
$$

be the vector running from the $m^{-}$point to the $m^{+}$point, and similarly for $\boldsymbol{v}_{\mathrm{t}}$ and $\boldsymbol{v}_{[\mathrm{Fe} / \mathrm{H}]}$, the weight $w$ is then calculated as

$$
w=\frac{\Delta u_{m} \Delta u_{t} \Delta u_{[\mathrm{Fe} / \mathrm{H}]}}{\boldsymbol{v}_{m} \cdot\left(\boldsymbol{v}_{\mathrm{t}} \times \boldsymbol{v}_{[\mathrm{Fe} / \mathrm{H}]}\right)}
$$

where the denominator is the volume of the parallelepiped spanned by the three vectors. We use these weights in calculating the weighted median and $1 \sigma$ confidence regions of each parameter chain.

\section{REFERENCES}

Albrecht, S., Winn, J. N., Johnson, J. A., et al. 2011, ApJ, 738, 50 Ammler-von Eiff, M., Santos, N. C., Sousa, S. G., et al. 2009, A\&A, 507, 523 Anderson, D. R., Collier Cameron, A., Delrez, L., et al. 2014, MNRAS, 445, 1114

Anderson, D. R., Collier Cameron, A., Gillon, M., et al. 2011a, A\&A, 534, A16

Anderson, D. R., Collier Cameron, A., Gillon, M., et al. 2012, MNRAS, 422, 1988

Anderson, D. R., Collier Cameron, A., Hellier, C., et al. 2011b, ApJL, 726, L19

Anderson, D. R., Collier Cameron, A., Hellier, C., et al. 2011c, A\&A, 531, A60
Anderson, D. R., Collier Cameron, A., Hellier, C., et al. 2015, A\&A, 575, A61 Anderson, D. R., Smith, A. M. S., Lanotte, A. A., et al. 2011d, MNRAS, 416, 2108

Bakos, G., Noyes, R. W., Kovács, G., et al. 2004, PASP, 116, 266

Bakos, G. Á., Csubry, Z., Penev, K., et al. 2013, PASP, 125, 154

Bakos, G. Á., Hartman, J. D., Bhatti, W., et al. 2015a, AJ, 149, 149

Bakos, G. Á., Hartman, J., Torres, G., et al. 2011, ApJ, 742, 116

Bakos, G. Á., Hartman, J. D., Torres, G., et al. 2012, AJ, 144, 19

Bakos, G. Á., Hartman, J. D., Torres, G., et al. 2016, arXiv:1606.04556

Bakos, G. Á., Howard, A. W., Noyes, R. W., et al. 2009a, ApJ, 707, 446

Bakos, G. Á., Pál, A., Torres, G., et al. 2009b, ApJ, 696, 1950

Bakos, G. Á., Penev, K., Bayliss, D., et al. 2015b, ApJ, 813, 111

Bakos, G. Á., Shporer, A., Pál, A., et al. 2007, ApJL, 671, L173

Bakos, G. Á., Torres, G., Pál, A., et al. 2010, ApJ, 710, 1724

Baraffe, I., Chabrier, G., \& Barman, T. 2008, A\&A, 482, 315

Barros, S. C. C., Faedi, F., Collier Cameron, A., et al. 2011a, A\&A, 525, A54

Barros, S. C. C., Pollacco, D. L., Gibson, N. P., et al. 2011b, MNRAS, 416, 2593

Barros, S. C. C., Pollacco, D. L., Gibson, N. P., et al. 2012, MNRAS, 419, 1248

Batygin, K., \& Stevenson, D. J. 2010, ApJL, 714, L238

Batygin, K., Stevenson, D. J., \& Bodenheimer, P. H. 2011, ApJ, 738, 1

Bayliss, D., Hartman, J. D., Bakos, G. Á., et al. 2015, AJ, 150, 49

Bayliss, D., Zhou, G., Penev, K., et al. 2013, AJ, 146, 113

Beatty, T. G., Pepper, J., Siverd, R. J., et al. 2012, ApJL, 756, L39

Becker, J. C., Vanderburg, A., Adams, F. C., Rappaport, S. A., \& Schwengeler, H. M. 2015, ApJL, 812, L18

Béky, B., Bakos, G. Á., Hartman, J., et al. 2011, ApJ, 734, 109

Bento, J., Schmidt, B., Hartman, J., et al. 2016, arXiv:1607.00688

Bhatti, W., Bakos, G. Á., Hartman, J. D., et al. 2016, arXiv:1607.00322

Bieryla, A., Collins, K., Beatty, T. G., et al. 2015, AJ, 150, 12

Bieryla, A., Hartman, J. D., Bakos, G. Á., et al. 2014, AJ, 147, 84

Bodenheimer, P., Lin, D. N. C., \& Mardling, R. A. 2001, ApJ, 548, 466

Boisse, I., Hartman, J. D., Bakos, G. Á., et al. 2013, A\&A, 558, A86

Borucki, W. J., Koch, D., Basri, G., et al. 2010, Sci, 327, 977

Bouchy, F., Hebb, L., Skillen, I., et al. 2010, A\&A, 519, A98

Bouchy, F., Hébrard, G., Udry, S., et al. 2009, A\&A, 505, 853

Brahm, R., Jordán, A., Bakos, G. Á., et al. 2016, AJ, 151, 89

Brahm, R., Jordán, A., Hartman, J. D., et al. 2015, AJ, 150, 33

Buchhave, L. A., Bakos, G. Á., Hartman, J. D., et al. 2010, ApJ, 720, 1118

Buchhave, L. A., Bakos, G. Á., Hartman, J. D., et al. 2011, ApJ, 733, 116

Buchhave, L. A., Latham, D. W., Johansen, A., et al. 2012, Natur, 486, 375

Burrows, A., Guillot, T., Hubbard, W. B., et al. 2000, ApJL, 534, L97

Burrows, A., Hubeny, I., Budaj, J., \& Hubbard, W. B. 2007, ApJ, 661, 502

Butler, R. P., Marcy, G. W., Williams, E., et al. 1996, PASP, 108, 500

Campo, C. J., Harrington, J., Hardy, R. A., et al. 2011, ApJ, 727, 125

Cardelli, J. A., Clayton, G. C., \& Mathis, J. S. 1989, ApJ, 345, 245

Chabrier, G. 2003, PASP, 115, 763

Charbonneau, D., Brown, T. M., Latham, D. W., \& Mayor, M. 2000, ApJL, 529, L45

Charbonnel, C., \& Palacios, A. 2004, in IAU Symp. 215, Stellar Rotation, ed. A. Maeder \& P. Eenens (San Francisco, CA: ASP), 440

Christensen-Dalsgaard, J., Kjeldsen, H., Brown, T. M., et al. 2010, ApJL, 713, L164

Christian, D. J., Gibson, N. P., Simpson, E. K., et al. 2009, MNRAS, 392, 1585

Christiansen, J. L., Ballard, S., Charbonneau, D., et al. 2010, ApJ, 710, 97

Ciceri, S., Mancini, L., Henning, T., et al. 2015, arXiv:1511.06305

Claret, A. 2004, A\&A, 428, 1001

Collier Cameron, A., Bouchy, F., Hébrard, G., et al. 2007, MNRAS, 375, 951

Collier Cameron, A., Guenther, E., Smalley, B., et al. 2010, MNRAS, 407, 507

Collins, K. A., Eastman, J. D., Beatty, T. G., et al. 2014, AJ, 147, 39

Dai, F., Winn, J. N., Arriagada, P., et al. 2015, ApJL, 813, L9

de Val-Borro, M., Bakos, G. Á., Brahm, R., et al. 2016, arXiv:1607.00006

Delrez, L., Santerne, A., Almenara, J.-M., et al. 2015, arXiv:1506.02471

Delrez, L., Van Grootel, V., Anderson, D. R., et al. 2014, A\&A, 563, A143

Demory, B.-O., \& Seager, S. 2011, ApJS, 197, 12

Dittmann, J. A., Close, L. M., Scuderi, L. J., Turner, J., \& Stephenson, P. C. 2012, NewA, 17, 438

Djupvik, A. A., \& Andersen, J. 2010, in Highlights of Spanish Astrophysics V, Astrophysics and Space Science Proceedings, ed. J. M. Diego et al. (Heidelberg: Springer-Verlag), 211

Droege, T. F., Richmond, M. W., Sallman, M. P., \& Creager, R. P. 2006, PASP, 118, 1666

Eastman, J. D., Beatty, T. G., Siverd, R. J., et al. 2016, AJ, 151, 45

Efron, B., \& Petrosian, V. 1999, J. Am. Stat. Assoc., 94, 824

Enoch, B., Anderson, D. R., Barros, S. C. C., et al. 2011a, AJ, 142, 86 
Enoch, B., Cameron, A. C., Anderson, D. R., et al. 2011b, MNRAS, 410, 1631 Enoch, B., Collier Cameron, A., \& Horne, K. 2012, A\&A, 540, A99 Espinoza, N., Bayliss, D., Hartman, J. D., et al. 2016, arXiv:1606.00023 Espinoza, N., \& Jordán, A. 2015, MNRAS, 450, 1879

Faedi, F., Barros, S. C. C., Anderson, D. R., et al. 2011, A\&A, 531, A40 Faedi, F., Pollacco, D., Barros, S. C. C., et al. 2013, A\&A, 551, A73 Fortney, J. J., Marley, M. S., \& Barnes, J. W. 2007, ApJ, 659, 1661 Fukui, A., Narita, N., Tristram, P. J., et al. 2011, PASJ, 63, 287 Fulton, B. J., Collins, K. A., Gaudi, B. S., et al. 2015, ApJ, 810, 30 Füresz, G. 2008, PhD thesis, Univ. Szeged

Geweke, J. 1992, in Bayesian Statistics 4, ed. J. M. Bernardo et al. (Oxford: Oxford Univ. Press)

Gibson, N. P., Pollacco, D., Simpson, E. K., et al. 2008, A\&A, 492, 603 Gibson, N. P., Pollacco, D., Simpson, E. K., et al. 2009, ApJ, 700, 1078 Gibson, N. P., Pollacco, D. L., Barros, S., et al. 2010, MNRAS, 401, 1917 Gillon, M., Anderson, D. R., Collier-Cameron, A., et al. 2013, A\&A, 552, A82 Gillon, M., Anderson, D. R., Collier-Cameron, A., et al. 2014, A\&A, 562, L3 Gillon, M., Anderson, D. R., Triaud, A. H. M. J., et al. 2009a, A\&A, 501, 785 Gillon, M., Doyle, A. P., Lendl, M., et al. 2011, A\&A, 533, A88 Gillon, M., Smalley, B., Hebb, L., et al. 2009b, A\&A, 496, 259 Girardi, L., Bertelli, G., Bressan, A., et al. 2002, A\&A, 391, 195 Gómez Maqueo Chew, Y., Faedi, F., Pollacco, D., et al. 2013, A\&A, 559, A36 Grunblatt, S. K., Huber, D., Gaidos, E. J., et al. 2016, arXiv:1606.05818 Hansen, B. M. S., \& Barman, T. 2007, ApJ, 671, 861

Hartman, J. D., Bakos, G. Á., Béky, B., et al. 2012, AJ, 144, 139

Hartman, J. D., Bakos, G. Á., Buchhave, L. A., et al. 2015a, AJ, 150, 197 Hartman, J. D., Bakos, G. Á., Kipping, D. M., et al. 2011a, ApJ, 728, 138 Hartman, J. D., Bakos, G. Á., Sato, B., et al. 2011b, ApJ, 726, 52 Hartman, J. D., Bakos, G. Á., Torres, G., et al. 2009, ApJ, 706, 785 Hartman, J. D., Bakos, G. Á., Torres, G., et al. 2011c, ApJ, 742, 59 Hartman, J. D., Bakos, G. Á., Torres, G., et al. 2014, AJ, 147, 128 Hartman, J. D., Bayliss, D., Brahm, R., et al. 2015b, AJ, 149, 166 Hartman, J. D., Bhatti, W., Bakos, G. Á., et al. 2015c, AJ, 150, 168 Hebb, L., Collier-Cameron, A., Loeillet, B., et al. 2009, ApJ, 693, 1920

Hebb, L., Collier-Cameron, A., Triaud, A. H. M. J., et al. 2010, ApJ, 708, 224 Hébrard, G., Collier Cameron, A., Brown, D. J. A., et al. 2013, A\&A, 549, A134

Hellier, C., Anderson, D. R., Cameron, A. C., et al. 2014, MNRAS, 440, 1982 Hellier, C., Anderson, D. R., Collier Cameron, A., et al. 2009a, Natur, 460, 1098

Hellier, C., Anderson, D. R., Collier Cameron, A., et al. 2010, ApJL, 723, L60 Hellier, C., Anderson, D. R., Collier Cameron, A., et al. 2011a, A\&A, 535, L7 Hellier, C., Anderson, D. R., Collier Cameron, A., et al. 2012, MNRAS, 426, 739

Hellier, C., Anderson, D. R., Collier Cameron, A., et al. 2015, AJ, 150, 18

Hellier, C., Anderson, D. R., Collier Cameron, A., et al. 2016, arXiv:1604. 04195

Hellier, C., Anderson, D. R., Collier-Cameron, A., et al. 2011b, ApJL, 730, L31

Hellier, C., Anderson, D. R., Gillon, M., et al. 2009b, ApJL, 690, L89

Henry, G. W., Marcy, G. W., Butler, R. P., \& Vogt, S. S. 2000, ApJL, 529, L41 Howard, A. W., Bakos, G. Á., Hartman, J., et al. 2012, ApJ, 749, 134

Huang, C. X., Hartman, J. D., Bakos, G. Á., et al. 2015, AJ, 150, 85

Huang, X., \& Cumming, A. 2012, ApJ, 757, 47

Ibgui, L., Burrows, A., \& Spiegel, D. S. 2010, ApJ, 713, 751

Ibgui, L., Spiegel, D. S., \& Burrows, A. 2011, ApJ, 727, 75

Isaacson, H., \& Fischer, D. 2010, ApJ, 725, 875

Johnson, J. A., Winn, J. N., Albrecht, S., et al. 2009a, PASP, 121, 1104

Johnson, J. A., Winn, J. N., Bakos, G. Á., et al. 2011, ApJ, 735, 24

Johnson, J. A., Winn, J. N., Cabrera, N. E., \& Carter, J. A. 2009b, ApJL, 692, L100

Johnson, J. A., Winn, J. N., Narita, N., et al. 2008, ApJ, 686, 649

Jordán, A., Brahm, R., Bakos, G. Á., et al. 2014, AJ, 148, 29

Joshi, Y. C., Pollacco, D., Collier Cameron, A., et al. 2009, MNRAS, 392,1532

Juncher, D., Buchhave, L. A., Hartman, J. D., et al. 2015, PASP, 127, 851

Kipping, D. M., Bakos, G. Á., Hartman, J., et al. 2010, ApJ, 725, 2017

Kipping, D. M., Hartman, J., Bakos, G. Á., et al. 2011, AJ, 142, 95

Knutson, H. A., Charbonneau, D., Burrows, A., O’Donovan, F. T., \& Mandushev, G. 2009, ApJ, 691, 866

Kovács, G., Bakos, G., \& Noyes, R. W. 2005, MNRAS, 356, 557

Kovács, G., Bakos, G. Á., Hartman, J. D., et al. 2010, ApJ, 724, 866

Kovács, G., Bakos, G. Á., Torres, G., et al. 2007, ApJL, 670, L41

Kovács, G., Zucker, S., \& Mazeh, T. 2002, A\&A, 391, 369

Krejčová, T., Budaj, J., \& Krushevska, V. 2010, CoSka, 40, 77

Kuhn, R. B., Rodriguez, J. E., Collins, K. A., et al. 2015, arXiv:1509.02323
Lagarde, N., Decressin, T., Charbonnel, C., et al. 2012, A\&A, 543, A108 Latham, D. W., Bakos, G. Á., Torres, G., et al. 2009, ApJ, 704, 1107

Lendl, M., Anderson, D. R., Collier-Cameron, A., et al. 2012, A\&A, 544, A72 Lendl, M., Triaud, A. H. M. J., Anderson, D. R., et al. 2014, A\&A, 568, A81 Liang, F., Paulo, R., Molina, G., Clyde, M. A., \& Berger, J. O. 2008, J. Am. Stat. Assoc., 103, 410

Lister, T. A., Anderson, D. R., Gillon, M., et al. 2009, ApJ, 703, 752

Liu, X., Burrows, A., \& Ibgui, L. 2008, ApJ, 687, 1191

Lloyd, J. P. 2011, ApJL, 739, L49

Lopez, E. D., \& Fortney, J. J. 2016, ApJ, 818, 4

Lucy, L. B., \& Sweeney, M. A. 1971, AJ, 76, 544

Mancini, L., Hartman, J. D., Penev, K., et al. 2015, A\&A, 580, A63

Mandel, K., \& Agol, E. 2002, ApJL, 580, L171

Mandushev, G., O’Donovan, F. T., Charbonneau, D., et al. 2007, ApJL, 667, L195

Mandushev, G., Quinn, S. N., Buchhave, L. A., et al. 2011, ApJ, 741, 114

Maxted, P. F. L., Anderson, D. R., Collier Cameron, A., et al. 2010a, PASP, 122,1465

Maxted, P. F. L., Anderson, D. R., Collier Cameron, A., et al. 2011, PASP, 123,547

Maxted, P. F. L., Anderson, D. R., Collier Cameron, A., et al. 2013, PASP, 125,48

Maxted, P. F. L., Anderson, D. R., Collier Cameron, A., et al. 2016, arXiv:1602.01740

Maxted, P. F. L., Anderson, D. R., Gillon, M., et al. 2010b, AJ, 140, 2007

Močnik, T., Anderson, D. R., Brown, D. J. A., et al. 2016, arXiv:1603.05638

Mohler-Fischer, M., Mancini, L., Hartman, J. D., et al. 2013, A\&A, 558, A55

Neveu-VanMalle, M., Queloz, D., Anderson, D. R., et al. 2014, A\&A, 572, A49

Noyes, R. W., Bakos, G. Á., Torres, G., et al. 2008, ApJL, 673, L79

Noyes, R. W., Hartmann, L. W., Baliunas, S. L., Duncan, D. K., \& Vaughan, A. H. 1984, ApJ, 279, 763

O’Donovan, F. T., Charbonneau, D., Mandushev, G., et al. 2006, ApJL, 651, L61

O'Rourke, J. G., Knutson, H. A., Zhao, M., et al. 2014, ApJ, 781, 109

Pál, A. 2012, MNRAS, 421, 1825

Pál, A., Bakos, G. Á., Torres, G., et al. 2008, ApJ, 680, 1450

Pál, A., Bakos, G. Á., Torres, G., et al. 2010, MNRAS, 401, 2665

Penev, K., Bakos, G. Á., Bayliss, D., et al. 2013, AJ, 145, 5

Penev, K. M., Hartman, J. D., Bakos, G. A., et al. 2016, arXiv:1606.00848

Pepper, J., Siverd, R. J., Beatty, T. G., et al. 2013, ApJ, 773, 64

Pollacco, D., Skillen, I., Collier Cameron, A., et al. 2008, MNRAS, 385,1576

Pollacco, D. L., Skillen, I., Collier Cameron, A., et al. 2006, PASP, 118, 1407

Pont, F., \& Eyer, L. 2004, MNRAS, 351, 487

Prša, A., Harmanec, P., Torres, G., et al. 2016, AJ, 152, 41

Queloz, D., Anderson, D. R., Collier Cameron, A., et al. 2010, A\&A, 517, L1

Quinn, S. N., Bakos, G. Á., Hartman, J., et al. 2012, ApJ, 745, 80

Rabus, M., Deeg, H. J., Alonso, R., Belmonte, J. A., \& Almenara, J. M. 2009 A\&A, 508, 1011

Rabus, M., Jordán, A., Hartman, J. D., et al. 2016, arXiv:1603.02894

Raetz, S., Mugrauer, M., Schmidt, T. O. B., et al. 2009, AN, 330, 475

Rodriguez, J. E., Colon, K. D., Stassun, K. G., et al. 2015, arXiv:1509.08953

Rouder, J. N., \& Morey, R. D. 2012, Multivariate Behavioral Research, 47, 877

Santerne, A., Hébrard, G., Deleuil, M., et al. 2014, A\&A, 571, A37

Santerne, A., Moutou, C., Tsantaki, M., et al. 2016, A\&A, 587, A64

Santos, N. C., Sousa, S. G., Mortier, A., et al. 2013, A\&A, 556, A150

Sato, B., Hartman, J. D., Bakos, G. Á., et al. 2012, PASJ, 64

Shporer, A., Bakos, G. Á., Bouchy, F., et al. 2009, ApJ, 690, 1393

Simpson, E. K., Faedi, F., Barros, S. C. C., et al. 2011, AJ, 141, 8

Siverd, R. J., Beatty, T. G., Pepper, J., et al. 2012, ApJ, 761, 123

Skillen, I., Pollacco, D., Collier Cameron, A., et al. 2009, A\&A, 502, 391

Smalley, B., Anderson, D. R., Collier Cameron, A., et al. 2010, A\&A, 520, A56

Smalley, B., Anderson, D. R., Collier Cameron, A., et al. 2011, A\&A, 526, A130

Smalley, B., Anderson, D. R., Collier-Cameron, A., et al. 2012, A\&A, 547, A61

Smith, A. M. S., Anderson, D. R., Armstrong, D. J., et al. 2014, A\&A, 570, A64

Smith, A. M. S., Anderson, D. R., Bouchy, F., et al. 2013, A\&A, 552, A120 Smith, A. M. S., Anderson, D. R., Collier Cameron, A., et al. 2012, AJ, 143, 81 Snaith, O., Haywood, M., Di Matteo, P., et al. 2015, A\&A, 578, A87

Southworth, J., Hinse, T. C., Dominik, M., et al. 2009a, ApJ, 707, 167

Southworth, J., Hinse, T. C., Jørgensen, U. G., et al. 2009b, MNRAS, 396,1023 
Sozzetti, A., Torres, G., Charbonneau, D., et al. 2007, ApJ, 664, 1190

Sozzetti, A., Torres, G., Charbonneau, D., et al. 2009, ApJ, 691, 1145

Spake, J. J., Brown, D. J. A., Doyle, A. P., et al. 2016, PASP, 128, 024401

Spiegel, D. S., \& Burrows, A. 2013, ApJ, 772, 76

Stempels, H. C., Collier Cameron, A., Hebb, L., Smalley, B., \& Frandsen, S. 2007, MNRAS, 379, 773

Street, R. A., Simpson, E., Barros, S. C. C., et al. 2010, ApJ, 720, 337

Szabo, G. M., Haja, O., Szatmary, K., Pal, A., \& Kiss, L. L. 2010, IBVS, 5919, 1

ter Braak, C. J. F. 2006, Statistics and Computing, 16, 239

Torres, G., Bakos, G. Á., Hartman, J., et al. 2010, ApJ, 715, 458

Torres, G., Bakos, G. Á., Kovács, G., et al. 2007, ApJL, 666, L121

Torres, G., Fischer, D. A., Sozzetti, A., et al. 2012, ApJ, 757, 161

Torres, G., Winn, J. N., \& Holman, M. J. 2008, ApJ, 677, 1324

Triaud, A. H. M. J., Anderson, D. R., Collier Cameron, A., et al. 2013, A\&A, 551, A80

Triaud, A. H. M. J., Queloz, D., Hellier, C., et al. 2011, A\&A, 531, A24

Tripathi, A., Winn, J. N., Johnson, J. A., et al. 2010, ApJ, 715, 421
Turner, O. D., Anderson, D. R., Collier Cameron, A., et al. 2015, arXiv:1509. 02210

Van Eylen, V., Albrecht, S., Gandolfi, D., et al. 2016, arXiv:1605.09180

Vogt, S. S., Allen, S. L., Bigelow, B. C., et al. 1994, Proc. SPIE, 2198, 362

Weinberg, M. D., Yoon, I., \& Katz, N. 2013, arXiv:1301.3156

Welsh, W. F., Orosz, J. A., Seager, S., et al. 2010, ApJL, 713, L145

West, R. G., Anderson, D. R., Gillon, M., et al. 2009, AJ, 137, 4834

West, R. G., Hellier, C., Almenara, J.-M., et al. 2016, A\&A, 585, A126

Winn, J. N., Holman, M. J., Carter, J. A., et al. 2009, AJ, 137, 3826

Winn, J. N., Howard, A. W., Johnson, J. A., et al. 2011, AJ, 141, 63

Winn, J. N., Johnson, J. A., Howard, A. W., et al. 2010, ApJ, 718, 575

Wu, Y., \& Lithwick, Y. 2013, ApJ, 763, 13

Yi, S., Demarque, P., Kim, Y.-C., et al. 2001, ApJS, 136, 417

Zacharias, N., Finch, C. T., Girard, T. M., et al. 2013, AJ, 145, 44 Zechmeister, M., \& Kürster, M. 2009, A\&A, 496, 577

Zhao, M., O'Rourke, J. G., Wright, J. T., et al. 2014, ApJ, 796, 115

Zhou, G., Bayliss, D., Hartman, J. D., et al. 2015, ApJL, 814, L16

Zhou, G., Bayliss, D., Penev, K., et al. 2014, AJ, 147, 144 